\title{
Classification of level of severity of Rheumatoid Arthritis using Machine Learning
} (Decision Tree)

\author{
By
}

Abiola Ogungbemile

B.Sc. Electrical Engineering, Clark Atlanta University

A thesis submitted to the Faculty of Graduate Studies and Research

In partial fulfilment of the requirements for the degree of

Master of Applied Science in Biomedical Engineering

\author{
Ottawa-Carleton Institute for Biomedical Engineering (OCIBME) \\ Department of Systems and Computer Engineering \\ Carleton University \\ Ottawa, Ontario, Canada, K1S 5B6
}

May 2011

(C) Copyright 2011, Abiola Ogungbemile 
Library and Archives

Canada

Published Heritage Branch

395 Wellington Street

Ottawa ON K1A ON4

Canada
Bibliothèque et

Archives Canada

Direction du

Patrimoine de l'édition

395 , rue Wellington

Ottawa ON K1A ON4

Canada
Your file Votre référence

ISBN: 978-0-494-81668-4

Our file Notre référence

ISBN: 978-0-494-81668-4
NOTICE:

The author has granted a nonexclusive license allowing Library and Archives Canada to reproduce, publish, archive, preserve, conserve, communicate to the public by telecommunication or on the Internet, loan, distribute and sell theses worldwide, for commercial or noncommercial purposes, in microform, paper, electronic and/or any other formats.

The author retains copyright ownership and moral rights in this thesis. Neither the thesis nor substantial extracts from it may be printed or otherwise reproduced without the author's permission.
AVIS:

L'auteur a accordé une licence non exclusive permettant à la Bibliothèque et Archives Canada de reproduire, publier, archiver, sauvegarder, conserver, transmettre au public par télécommunication ou par l'Internet, prêter, distribuer et vendre des thèses partout dans le monde, à des fins commerciales ou autres, sur support microforme, papier, électronique et/ou autres formats.

L'auteur conserve la propriété du droit d'auteur et des droits moraux qui protège cette thèse. $\mathrm{Ni}$ la thèse ni des extraits substantiels de celle-ci ne doivent être imprimés ou autrement reproduits sans son autorisation.
In compliance with the Canadian Privacy Act some supporting forms may have been removed from this thesis.

While these forms may be included in the document page count, their removal does not represent any loss of content from the thesis.
Conformément à la loi canadienne sur la protection de la vie privée, quelques formulaires secondaires ont été enlevés de cette thèse.

Bien que ces formulaires aient inclus dans la pagination, il n'y aura aucun contenu manquant.

\section{Canadä}


The undersigned recommend to

the Faculty of Graduate Studies and Research

acceptance of the thesis

\title{
Classification of the level of severity of Rheumatoid Arthritis using Machine Learning (Decision Tree)
}

\author{
submitted by \\ Abiola Ogungbemile, \\ B.Sc. Electrical Engineering, Clark Atlanta University \\ in partial fulfillment of the requirements for \\ the degree of Master of Applied Science in Biomedical Engineering
}

Chair, Dr. Howard Schwartz, Department of Systems and Computer Engineering

Thesis Supervisor, Dr. Monique Frize

Carleton University

May 2011 


\section{ABSTRACT}

This research is a continuation of earlier work, which assessed the use of thermal infrared (TIR) imaging systems as a potential tool for physicians to diagnose and manage Rheumatoid Arthritis (RA). From that work, TIR images were taken of 18 control subjects and 13 patients diagnosed with RA by a rheumatologist. However, in other to further explore and better improve classification 10 additional control subjects and 13 additional patients were recruited. Temperature measurements were extracted from all the joints in the hand, wrist, elbow, ankle, feet and knee images and statistical tests were done to show which joints were the best indicators of RA and good discriminators between the patients and the control subjects. It was established that the $2^{\text {nd }}$ metacarpophalangeal (MCP), the $3^{\text {rd }}$ metacarpophalangeal (MCP), the ankle and the knee were the joints that showed the greatest statistical difference between control subjects and patients. The dataset series were submitted to the C.5 software to generate a classifier (decision tree). The Min and Max datasets were the best in classifying RA with a sensitivity of $96 \%$ and a specificity of $92 \%$. The $2^{\text {nd }}$ and $3^{\text {rd }}$ MCPs proved to be the best joints to classify RA using the Min and Max dataset series confirming the statistical results from earlier work; where these same joints were concluded to be the best indicators of RA. The classifier was used to classify the level of severity of the patient group into LOW, MEDIUM and HIGH classes' successfully. 


\section{ACKNOWLEDGEMENTS}

First and foremost I would like to give thanks to God for the start and completion of this work, though the road was tumultuous He saw me through. Even when I couldn't see the vision or the end of the road, His word kept me going. All honour, glory and thanks belong to Him that established the earth and everything that is in it.

I would also like to thank my parents, Mr. and Mrs. Olusola Ogungbemile, for their constant support and encouragement throughout my life and for teaching and instilling in me the values that have shaped me and helped me be the best that I can be.

I would also like to thank my Supervisor, Dr. Monique Frize, for all her support throughout this journey, for extending herself to me even outside the boundaries of educational confines. Also I would like to thank her for setting an example to follow and for being a role model to women in Engineering; especially me.

I would like to thank the love of my life, "Toto" for always being there and loving me and showing me a new experience every day in love. For helping me believe in myself and my abilities and never letting me quit. Your love is that which strengthens me. I love you forever and always.

I would like to thank my brothers, Olaolu, Oladapo, Oladiran and Olayiwola Ogungbemile for being my support system through this whole process. For loving me even on days when I was erratic in my ways. And also my nephews - Anand and Femi, for bringing so much joy to my life and also to Vanessa. 
Special thanks to my pastors, for their prayers and encourage at a time when it seemed like all hope was lost. Their intercession on my behalf is greatly appreciated more than words are adequate enough to express. Also to Alice, Tomisin, John, and my entire church family for making my experience in Ottawa an amazing one and the exposure to many different things I would not have been otherwise privileged to experience.

Lastly I would like to thank Dr. Karsh for his contributions and the 26 patients and 28 control subjects that selflessly gave of their time for the continuation of this research. Your selfless act has helped advance research into early diagnosis of this disease that has no cure or known cause and has given reason to hope for the future of TIR imaging in RA diagnosis. 


\section{TABLE OF CONTENTS}

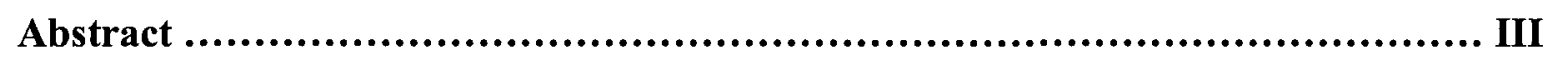

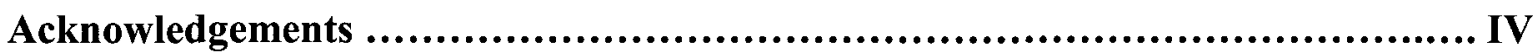

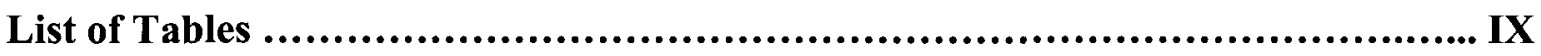

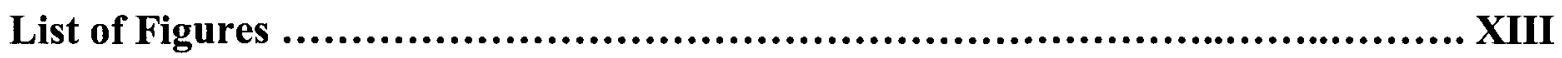

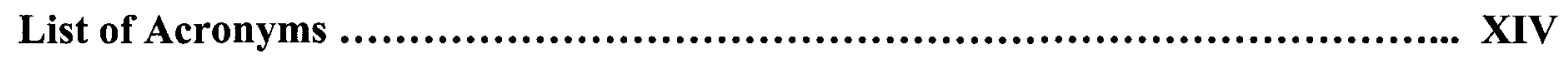

CHAPTER 1: INTRODUCTION .................................................. 1

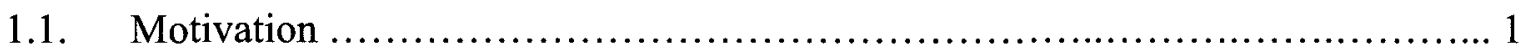

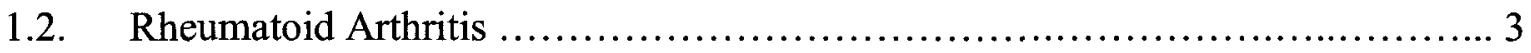

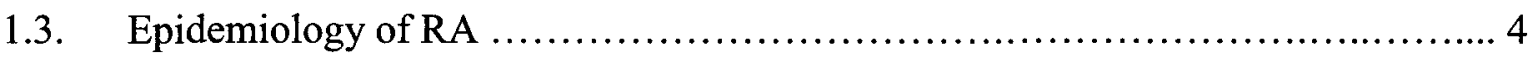

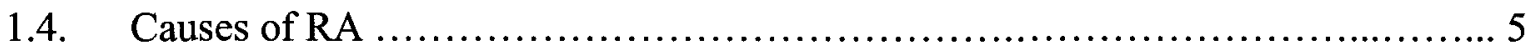

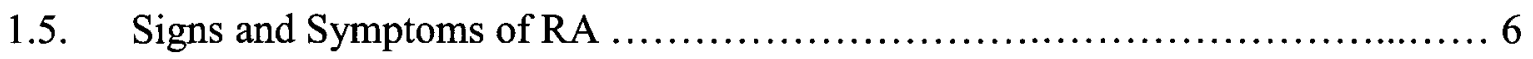

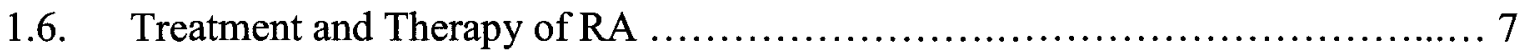

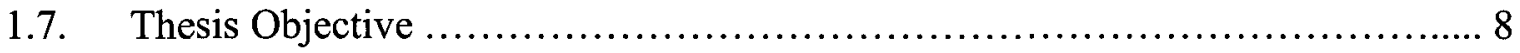

1.8. Thesis Outline ........................................................... 11

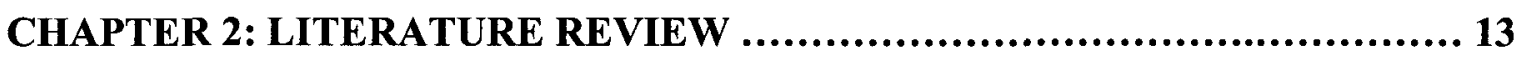

2.1. Medical Evaluation of Patient joints .................................... 13

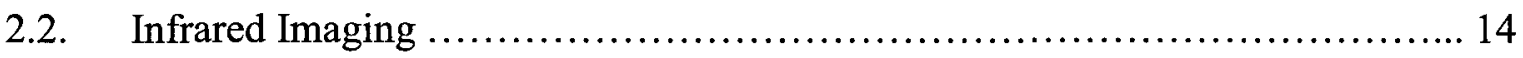

2.3. Past clinical applications of medical Infrared imaging ....................... 15

2.4. Applications of TIR imaging in RA ........................................ 16

2.5. Pattern classification methods ............................................... 18

2.5.1. Decision Trees .............................................................. 19

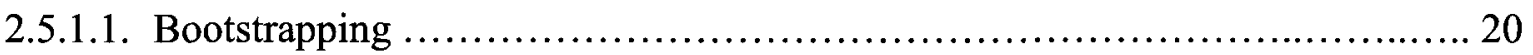

2.5.2. Ensemble Decision Tree Classifier ........................................... 20

2.6. Performance measures of classification methods ............................. 21

2.6.1. Confusion Datasets (Contingency Table) ...................................... 21

2.6.2. Correct Classification Rate (CCR) .......................................... 22

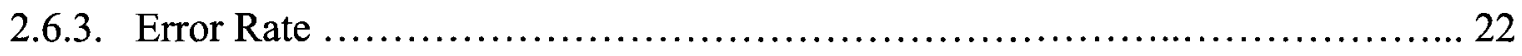

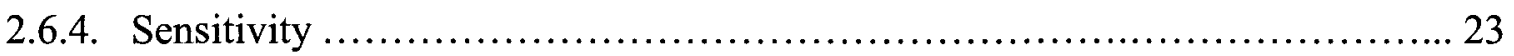

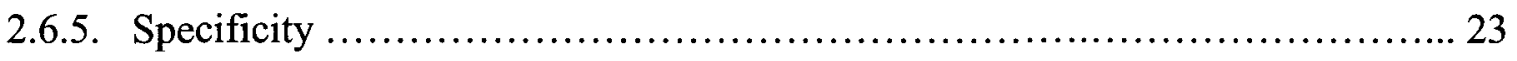

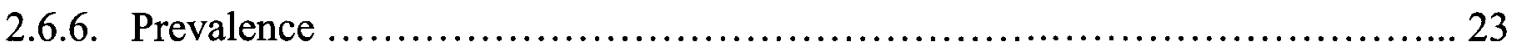

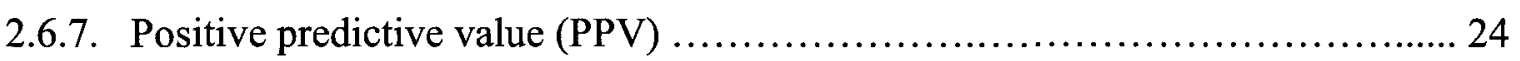




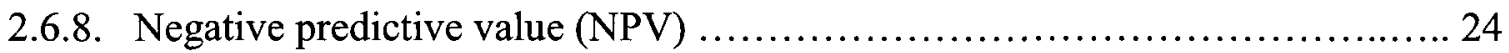

2.6.9. Receiver operating characteristics (ROC) curve .......................... 25

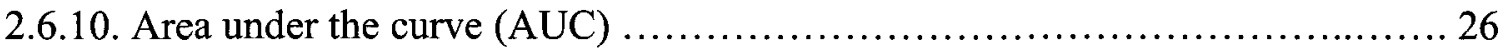

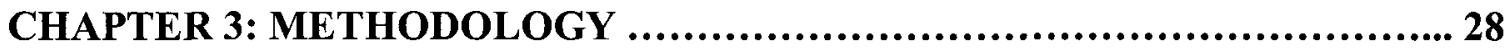

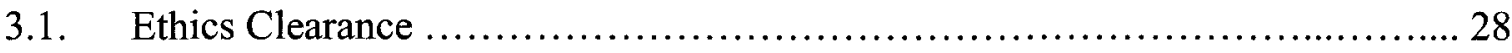

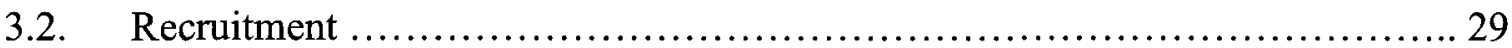

3.3. Collection of data .............................................................. 31

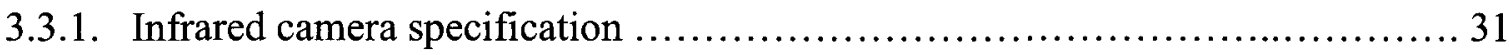

3.3.2. Infrared camera and recording settings ...................................... 31

3.3.3. Medical thermal infrared imaging protocol ................................. 32

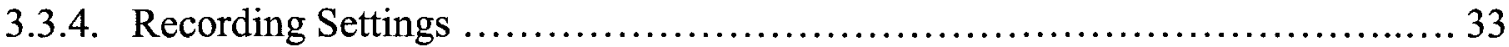

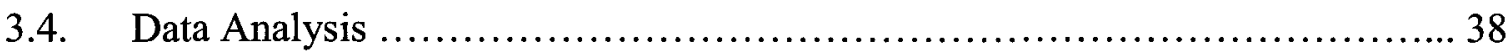

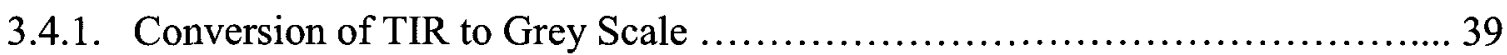

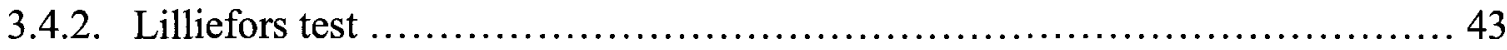

3.4.3. Kruskal-Wallis and Ranksum test ...................................... 44

3.5. Thesis Classification Methodology ............................................. 45

3.5.1. Accomplishing Objectives $\# 2$............................................. 45

3.5.2. Accomplishing Objective $\# 3$........................................... 46

3.5.3. Accomplishing Objective \#4 ................................................ 46

3.6. Decision Tree input Variables .......................................... 47

3.6.1. Input Variables ....................................................... 47

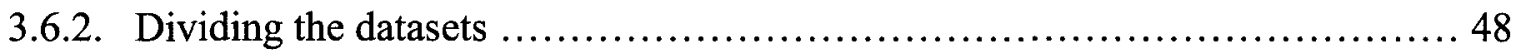

3.6.3. Ensemble Decision Tree .................................................. 49

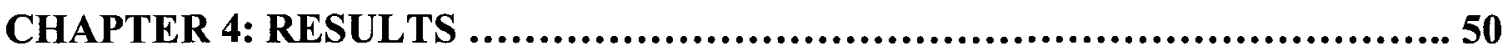

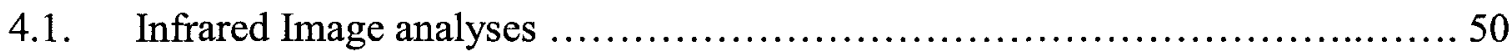

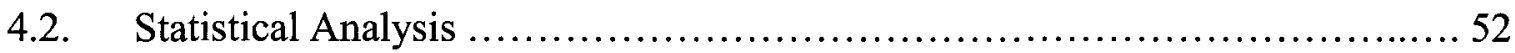

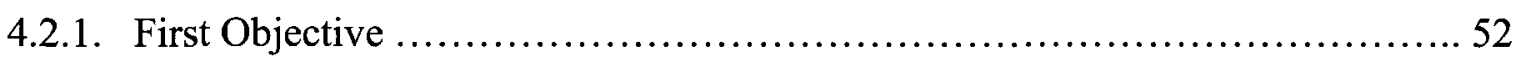

4.3. Classification \& Analysis ................................................. 58

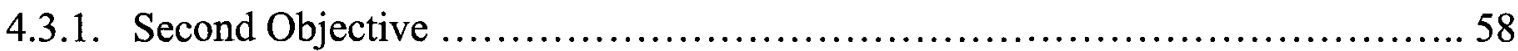

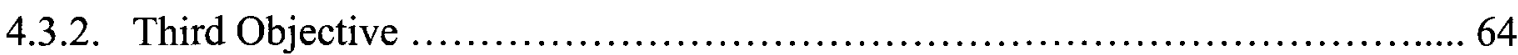

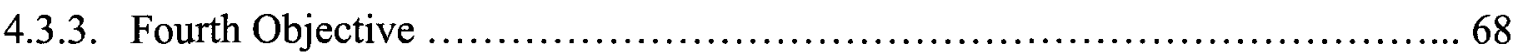

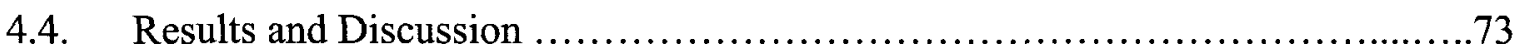

CHAPTER 5: CONCLUSIONS................................................. 75

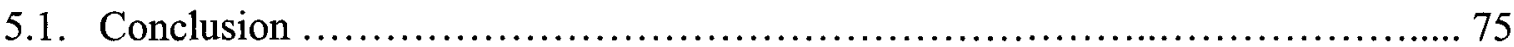

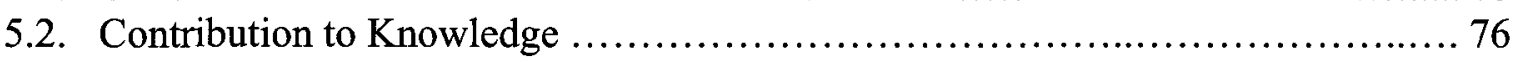

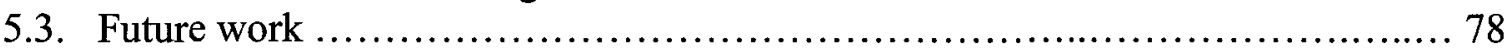




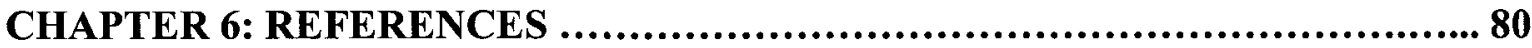

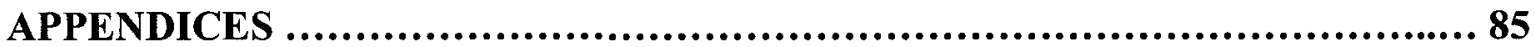

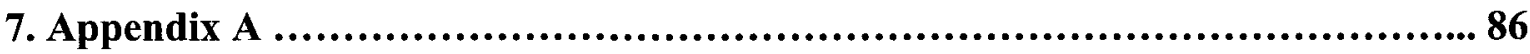

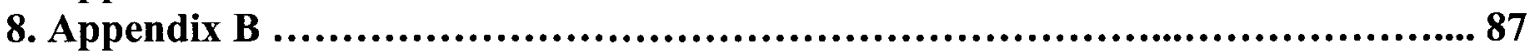

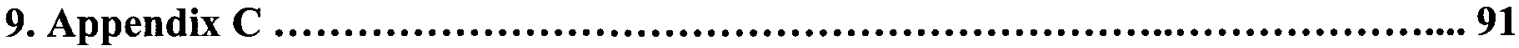

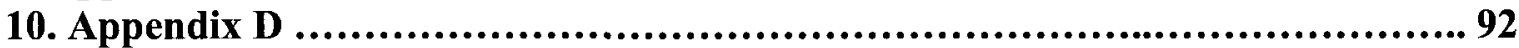

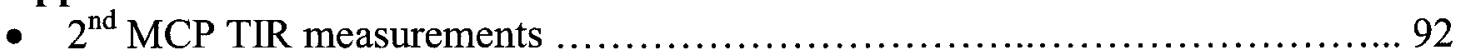

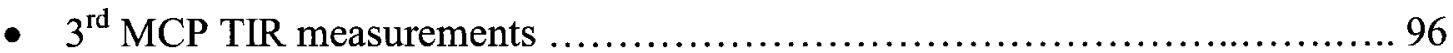

- Ankle TIR measurements ....................................................... 100

- Knee TIR measurements ..................................................... 104

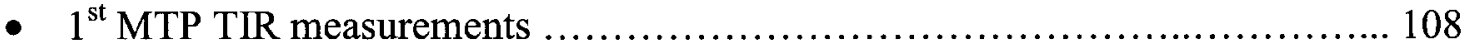

11. Appendix E .................................................................... 112

- Decision Tree Results for presence or absence of RA ....................... 112

- Decision Tree Results for elimination of variables (joints) ..................... 123

- Decision Tree Results for level of RA activity ................................. 126

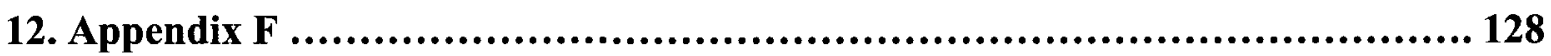

- Official Rheumatologist evaluation sheet.................................... 128

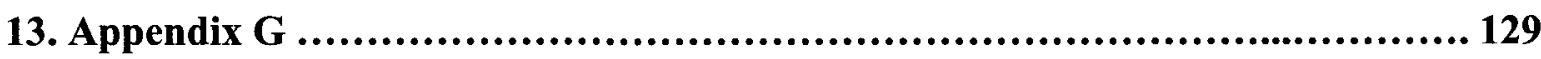

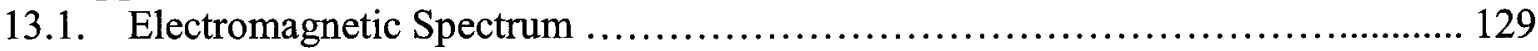

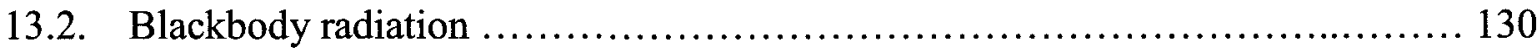

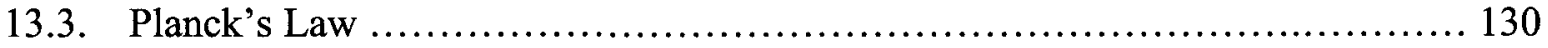

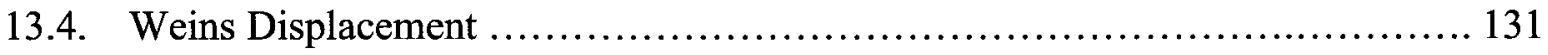

13.5. Stephan Boltzmann's Law ............................................... 133 


\section{LIST OF TABLES}

Table 1-1: American College of Rheumatology's Criteria for the Classification of Rheumatoid Arthritis (Arnett 1998, 315-324)

Table 2-1: A 2 x 2 Contingency Table

Table 2-2: AUC index and its effectiveness for discrimination (Fawcett, 2006)

Table 3-1: Grey Level Thermal Infrared Views of the Elbow (Adea, 2009)

Table 3-2: Grey Level Thermal Infrared Views of the Wrist and Hand (Adea, 2009)

Table 3-3: Grey Level Thermal Infrared Views of the Knee (Adea, 2009)

Table 3-4: Grey Level Thermal Infrared Views of the Ankle and Foot (Adea, 2009)

Table 3-5: Regions of Interests Manually Selected for the 2nd and 3rd MCPS, the 2nd and 3rd PIPs, the Wrist and the Knee (Adea, 2009)

Table 4-1: Average values for each dataset of every joint for the patient and control groups

Table 4-2: MATLAB Lilliefors Test Results for Randomly Selected Datasets

Table 4-3: MATLAB's Ranksum Test Results Comparing the Datasets of the Control and the Patient Groups

Table 4-4: Min and Max dataset Decision Tree result (Using 13 patients and 16 control subjects)

Table 4-5: Table showing the results of the DT combination of 12 different dataset series (Using 13 patients and 16 control subjects)

Table 4-6: Min and Max dataset Decision Tree result (Using 26 patients and 26 control subjects)

Table 4-7: Table showing the results of the DT combination of 12 different dataset series (Using 26 patients and 26 control subjects)

Table 4-8: Min and Max dataset Decision Tree result for Knee, 2nd MCP and 3rd MCP (Using 26 patients and 26 control subjects)

Table 4-9: Min and Max dataset Decision Tree result for different joint combinations (Using 26 patients and 26 control subjects)

Table 4-10: Table showing the results of the DT combination of 12 different dataset series for the Knees, 2nd MCP and 3rd MCP (Using 26 patients and 26 control subjects) 
Table 4-11: Min and Max dataset Decision Tree result for the 3rd MCP (Using 26 patients and 26 control subjects)

Table 4-12: Using the Min and Max dataset, table showing the specificity and sensitivity of the DT (Using 26 patients and 26 control subjects)

Table 4-13: Min and Max dataset Decision Tree result for level of severity of RA (Using 26 patients and 26 control subjects)

Table 4-14: Table showing the results of the DT combination of 12 different dataset series for the level of severity (Using 26 patients and 26 control subjects)

Table 4-15: Table showing the results of the classifier and the rheumatologists' evaluation Table 4-16: Contingency table for Min and Max dataset using the knee, ankle, $2^{\text {nd }}$ MCP and $3^{\text {rd }}$ MCP

Table 4-17: Confusion matrix for the Min and Max dataset

Table 10-1: Raw Data Measurements Extracted from the Control Group's $2^{\text {nd }}$ MCP Joint

Table 10-2: Raw Data Measurements Extracted from the Patient Group's $2^{\text {nd }}$ MCP Joint

Table 10-3: Raw Data Measurements Extracted from the Control Group's $3^{\text {rd }}$ MCP Joint

Table 10-4: Raw Data Measurements Extracted from the Patient Group's $3^{\text {rd }}$ MCP Joint

Table 10-5: Raw Data Measurements Extracted from the Control Group's Ankle Joint

Table 10-6: Raw Data Measurements Extracted from the Patient Group's Ankle Joint

Table 10-7: Raw Data Measurements Extracted from the Control Group's Knee Joint

Table 10-8: Raw Data Measurements Extracted from the Patient Group's Knee Joint

Table 10-9: Raw Data Measurements Extracted from the Control Group's $1^{\text {st }}$ MTP Joint

Table 10-10: Raw Data Measurements Extracted from the Patient Group's $1^{\text {st }}$ MTP Joint

Table 10-11: Raw Data Measurements Extracted from the Control Group's Elbow Joint

Table 10-12: Raw Data Measurements Extracted from the Patient Group's Elbow Joint

Table 11-1: Min/Max and Median/Max Decision Tree result (Using 13 patients and 16 control subjects)

Table 11-2: Mode/Min and Median/Min Decision Tree result (Using 13 patients and 16 control subjects) 
Table 11-3: Mode/Min and Mean/Min Decision Tree result (Using 13 patients and 16 control subjects)

Table 11-4: Mode/Max and Median/Max Decision Tree result (Using 13 patients and 16 control subjects)

Table 11-5: Min/Max and Median/Min Decision Tree result (Using 13 patients and 16 control subjects)

Table 11-6: Min/Max and Mean/Min Decision Tree result (Using 13 patients and 16 control subjects)

Table 11-8: Median/Max and Mode/Min Decision Tree result (Using 13 patients and 16 control subjects)

Table 11-9: Min/Max and Mode/Min Decision Tree result (Using 13 patients and 16 control subjects)

Table 11-10: Mode/Min and Median/Max Decision Tree result (Using 13 patients and 16 control subjects)

Table 11-11: Mode/Max and Mean/Max Decision Tree result (Using 13 patients and 16 control subjects)

Table 11-12: Min/Max and Median/Max Decision Tree result (Using 26 patients and 26 control subjects)

Table 11-13: Mode/Min and Median/Min Decision Tree result (Using 26 patients and 26 control subjects)

Table 11-14: Mode/Min and Mean/Min Decision Tree result (Using 26 patients and 26 control subjects)

Table 11-15: Mode/Max and Median/Max Decision Tree result (Using 26 patients and 26 control subjects)

Table 11-16: Min/Max and Median/Min Decision Tree result (Using 26 patients and 26 control subjects)

Table 11-17: Min/Max and Mean/Min Decision Tree result (Using 26 patients and 26 control subjects)

Table 11-18: Median/Max and Mode/Min Decision Tree result (Using 26 patients and 26 control subjects) 
Table 11-19: Mean/Max and Min/Max Decision Tree result (Using 26 patients and 26 control subjects)

Table 11-20: Min/Max and Mode/Min Decision Tree result (Using 26 patients and 26 control subjects)

Table 11-21: Mode/Max and Median/Max Decision Tree result (Using 26 patients and 26 control subjects)

Table 11-22: Mode/Max and Mean/Max Decision Tree result (Using 26 patients and 26 control subjects)

Table 11-23: Mode/Min and Median/Min Decision Tree result for Knee, $2^{\text {nd }}$ MCP and $3^{\text {rd }}$ MCP combination (Using 26 patients and 26 control subjects)

Table 11-24: Mode/Min and Median/Max Decision Tree result for Knee, 2nd MCP and 3rd MCP combination (Using 26 patients and 26 control subjects)

Table 11-25: Mode/Max and Mean/Max Decision Tree result for Knee, 2nd MCP and 3rd MCP combination (Using 26 patients and 26 control subjects)

Table 11-26: Mean/Max and Mode/Min Decision Tree result for Knee, 2nd MCP and 3rd MCP combination (Using 26 patients and 26 control subjects)

Table 11-27: Min/Max and Median/Max Decision Tree result for $3^{\text {rd }}$ MCP (Using 26 patients and 26 control subjects)

Table 11-28: Min/Max and Mean/Min Decision Tree result for $3^{\text {rd }}$ MCP (Using 26 patients and 26 control subjects)

Table 11-29: Min/Max and Median/Max Decision Tree result of level of severity (Using 26 patients and 26 control subjects)

Table 11-30: Median/Max and Mode/Min Decision Tree result of level of severity (Using 26 patients and 26 control subjects)

Table 11-31: Min/Max and Median/Min Decision Tree result of level of severity (Using 26 patients and 26 control subjects)

Table 11-32: Min/Max and Mode/Min Decision Tree result of level of severity (Using 26 patients and 26 control subjects) 


\section{LIST OF FIGURES}

Fig. 2-1: Preliminary sample model of the proposed decision tree for RA diagnosis.

Fig. 2-2: An ROC curve and the different points of significance (created from Fawcett 2006).

Fig. 3-1: A320 Infrared Camera from FLIR Systems (FLIR Systems, 2007)

Fig. 13-1: Electromagnetic Spectrum (FLIR Systems, 2007)

Fig. 13-2: Emissive Power of a Blackbody at Different Temperatures and Wavelengths according to Planck's Radiation Law (FLIR Systems, 2007)

Fig. 13-3: Planckian Curves Plotted on Semilog Scale (FLIR Systems, 2007) 


\section{LIST OF ACRONYMS}

$\begin{array}{ll}\text { ANN } & \text { Artificial Neural Network } \\ \text { AUC } & \text { Area Under the Curve } \\ \text { ATR } & \text { Automated Target Recognition } \\ \text { CCR } & \text { Correct Classification Rate } \\ \text { DIP } & \text { Distal InterPhalangeal } \\ \text { DMARDs } & \text { Disease-Modifying Anti-Rheumatic Drugs } \\ \text { DT } & \text { Decision Tree } \\ \text { FN } & \text { False Negative } \\ \text { FP } & \text { False Positive } \\ \text { HDI } & \text { Heat Distribution Index } \\ \text { HRUS } & \text { High Resolution Ultra Sound } \\ \text { IR } & \text { InfraRed } \\ \text { MCP } & \text { MetaCarpoPhalangeal } \\ \text { MIRG } & \text { Medical Information-technologies Research Group } \\ \text { MRI } & \text { Magnetic Resonance Imaging } \\ \text { MTP } & \text { MetaTarsal Phalangeal } \\ \text { NPV } & \text { Negative Predictive Value } \\ \text { NSAIDs } & \text { NonSteroidal Anti-Inflammatory Drugs } \\ \text { OHREB } & \text { Ottawa Hospital Research Ethics Board } \\ \text { PPV } & \text { Positive Predictive Value } \\ \text { RA } & \text { Receiver Operating Characteristic } \\ \text { ROC } & \text {. }\end{array}$


STD

TI

TIR

TN

TP

WHO
Standard Deviation

Thermal Index

Thermal InfraRed

True Negative

True Positive

World Health Organization 


\section{CHAPTER ONE}

\section{INTRODUCTION}

This chapter gives background information on the motivation behind this thesis; it also gives an introductory look into Rheumatoid Arthritis and discusses the disease, its causes, the signs \& symptoms and also available/current treatment \& therapy options. This detailed introduction is necessary in order to stress the importance of using thermal imaging as a potentially established diagnostic tool.

\subsection{Motivation}

From previous work (Adéa 2009), the use of thermal imaging as a tool to aid in the diagnosis and measurement of the severity of RA has proven to be effective. This is why the continuation of research work in this area is important. During flare up periods with this disease, there is always inflammation, which sometimes makes clinical assessment almost always impossible because the patient is in too much pain. But with the use of thermal imaging, this is a non-invasive tool that has shown potential to make assessment and diagnosis of RA easier and possibly earlier in the evolution of the condition. The progress that is continually made by the use of the technique makes it a viable diagnostic tool in the foreseeable future in Canada, the US and elsewhere. RA has a significant impact on health-related quality of life, and is associated with increased health-care costs and an increased mortality rate when affected patients are compared with the general population.

Rheumatoid arthritis is a debilitating disease that, if diagnosed early can be properly managed, controlling the disease activity, which is the only prevention strategy against 
permanent joint damage or severe deformity available at the moment. There are available modern treatment methods which are effective at controlling disease activity and reducing long term disability (Woolf \& Pfleger, 2003). It is imperative to identify patients early so that control of inflammation can prevent joint destruction and disability (Mody \& Cardiel, 2008). The etiology of this disease currently still remains unknown, which makes anyone from different walks of life susceptible to the disease. Therefore developing a diagnostic tool that is easy to use, non-invasive and cost effective bridges the gap and makes early diagnosis possible for everybody. There are limitless potentials and upsides to the development of this technology; it can also be used as a potential Telemedicine tool, and thereby offering diagnostic options to those who live in remote areas where the community cannot afford sophisticated equipments such as magnetic resonance imaging (MRI) and high resolution ultrasound (HRUS). Due to the inexpensive nature of this technology, its use can also be extended to developing countries, where there are currently no diagnostic tools for RA due to the expensive nature of some of the current imaging tests being used such as MRI and HRUS.

The successful implementation of this technique as a potential diagnostic tool would help to reduce the wait times for the current gold standard methods of diagnosing of RA and allow these tools to be used for the diagnosis of other diseases. This technology has a potential to aid rheumatologists in the diagnosis of RA in cases where they are confronted with limited to no physical manifestation of the disease. One of the major limitations rheumatologists face is the inability to diagnose RA in cases where there are no physical manifestations such as swelling, or inflammation. TIR imaging could make a diagnosis possible without physical symptoms because this is a measure of temperature readings over specific joints that have been shown to be indicators of RA (Adea, 2009). One characteristic of RA is inflammation in the affected joints, 
and these inflammations manifest themselves as abnormal increases in the temperature around these joints, which could be easily seen by the thermal imaging but not by the naked eye.

\subsection{Rheumatoid Arthritis}

This is an autoimmune disease associated with a chronic, inflammatory, systemic effect, which affects the musculoskeletal system. This disease is often progressive in nature and results in reduced physical function, pain, fatigue and joint damage (Uhlig et al, 2010). It is also characterized by a distinctive pattern of bone and joint destruction. The disease is manifested when the immune system, which is normally supposed to protect by fighting infections and diseases combats the body. During the flare-up, the immune system attacks the tissue that lines the joints, which causes pain, swelling, stiffness in those joints and affects their proper functioning. Over a period of time, RA begins to cause damage to bone and cartilage within the joints and weakens muscles and tendons that support the joints, and ultimately leads to joint destruction (NCBI, 2009).

It often begins in between the ages of 25 and 55 years old, however older people are more prone to this disease. It is also more prominent in women than in men, the statistic shows that it occurs in twice as many women as men. RA tends to run in families; research has been able to establish a genetic connection, showing that the genetic contribution to susceptibility is an estimated $60 \%$. There has also been a link to shared human leukocyte antigen- DRBI alleles, which is associated with RA and has been shown to probably play a role in determining the severity of the disease than susceptibility to it (Woolf \& Pfleger, 2003). All the joints in the body are susceptible to RA, but previous studies have shown that it is most common in the wrists and finger. It usually occurs in a symmetric pattern, where if it is present in one hand, it also affects 
the other. This is one of the distinctive differences between RA and osteoarthritis, the latter occurring in an asymmetric pattern.

RA usually beings in smaller joints of the hands and the feet, then later spreads to larger joints. It is marked by persistent systemic inflammation and tissue damage (Costenbader et al, 2010).The inflamed joint lining or synovium extends and then erodes the articular cartilage and bone, causing joint deformity and progressive physical disability (Symmons et al, 2006). Due to the progressive nature of this disease, research has shown that it can place a substantial burden on those afflicted and their families. There is no cure for RA currently, so patients are subjected to living their lives around the disease, which has shown to have detrimental effects on the quality of life of those affected. Though there are modern treatment options available and there has been a reduction in the mortality rate due to RA, treatment options and therapy can be sometimes painful and there have been reported complications that affect areas of normal living such as physical, psychological and social functioning (Marra et al, 2005).

\subsection{Epidemiology of $R A$}

Past research has shown that there was no evidence of RA in Europe, Asia, or Africa before the $17^{\text {th }}$ century, supporting the ideology that RA is a "modern disease" that can be attributed to an environmental (infectious) factor as its cause. It is believed by many that RA originated from North America, and then spread to Europe and other parts of the world (AbdelNasser et al, 1997), due to its high incidence rate among North Americans. Recent reports support this theory because till date, the highest occurrence rate can be found in Native Americans. 300000 Canadians, 2.1 million Americans and approximately 260000 French citizens are currently diagnosed with RA (Adea, 2009). There is no doubt about the worldwide 
impact of this disease on the population. The disease is known to occur in all populations, although much of the work on the impact of the disease on functional disability and mortality has been done in the industrialized world (Kalla \& Tikly, 2003)

According to reports, the rates of incidence in North America and Northern Europe ranges from $20-50$ cases per 100,000 population, and the prevalence at $0.5-1.1 \%$. In Southern Europe, however, the range is between 9 - 24 cases per 100,000, with a lower prevalence at 0.3 $-0.7 \%$ (Toban et al, 2010). The prevalence of RA is estimated to be $0.5-1.0 \%$ worldwide. However, considerable variation exists among ethnicities, with a higher prevalence in populations of European ancestry than those of Asian ancestry (Kochi et al, 2009). The numbers for the specific incidence rate in developing countries are not known at this time. However, there have been epidemiological studies done in developing countries that have shown a lower but still important prevalence in different regions when compared to that in Caucasians, it is also suspected that RA probably starts at a younger age in developing countries. The incidence and prevalence of RA generally rises with increasing age until about age 70, then patients start to decline.

\subsection{Causes of $R A$}

The etiology of RA is still unknown, however there are several factors believed to be involved in the development of RA, which includes but are not limited to environmental (infectious), genetic and hormonal factors. Some studies have also shown that smoking, obesity, an adverse pregnancy outcome, age and also sex, with the female gender twice as susceptible to the disease as the male gender, are contributing factors. Environmental and immunogenetic 
factors play a leading role in these multi-causal and often autoimmune disorders. Several amongst them are lifestyle disorders (Chopra \& Abdel-Nasser, 2008).

\subsection{Signs \& Symptoms}

During the early stages of rheumatoid arthritis, the disease tends to affect smaller joints first, the wrists, hands, ankles and feet, as the disease progresses and spreads, larger joints become affected- the shoulders, elbows, knees, hips, and neck. In the case of RA, symptoms occur symmetrically, in the same joints on both sides of your body. This is the most important distinguishing factor between Osteoarthritis and RA. The signs and symptoms may sometimes vary in the level of severity and may even come and go depending on the individual. Some of the signs and symptoms of RA may include, but are not limited to, joint pain, joint swelling, joint tenderness, red and puffy hands, firm bumps of tissue under the skin of the arms (rheumatoid nodules), fatigue, morning stiffness, which may last for hours, fever and weight loss. There are also periods of increased disease activity -called flare-ups or flares, alternate with periods of relative remission, during which the swelling, pain, difficulty sleeping and weakness fade or disappear.

There is a classification method which is the gold stand for diagnosing RA, which was established in 1987, by the American college of Rheumatology (ACR), Table 1 shows the classification criteria 
Table 1-1: American College of Rheumatology's Criteria for the Classification of Rheumatoid Arthritis (Arnett 1998, 315-324)

\begin{tabular}{|c|c|c|}
\hline \multicolumn{2}{|c|}{ Criterion } & \multirow{2}{*}{$\begin{array}{l}\text { Definition } \\
\text { Morning stiffness in and around the joints, lasting at } \\
\text { least } 1 \text { hour before maximal improvement }\end{array}$} \\
\hline (1) & Morning stiffness & \\
\hline (2) & $\begin{array}{l}\text { Arthritis of three or more joint } \\
\text { areas }\end{array}$ & $\begin{array}{l}\text { At least three joint areas simultaneously have had soft- } \\
\text { tissue swelling or fluid (not overgrowth alone) } \\
\text { observed by a physician. The } 14 \text { possible areas are } \\
\text { right or left PIP, MCP, wrist, elbow, knee, ankle and } \\
\text { MTP joints. }\end{array}$ \\
\hline (3) & Arthritis of hand joints & $\begin{array}{l}\text { At least one area swollen (as defined above) in a wrist, } \\
\text { MCP or PIP joint }\end{array}$ \\
\hline (4) & Symmetric arthritis & $\begin{array}{l}\text { Simultaneous involvement of the same joint areas (as } \\
\text { defined in 2) on both sides of the body (bilateral } \\
\text { involvement of PIPs, MCPs or MTPs is acceptable } \\
\text { without absolute symmetry). }\end{array}$ \\
\hline (5) & Rheumatoid nodules & $\begin{array}{l}\text { Subcutaneous nodules, over bony prominences, or } \\
\text { extensor or juxta-articular regions, observed by a } \\
\text { physician. }\end{array}$ \\
\hline (6) & Serum rheumatoid factor & $\begin{array}{l}\text { Demonstration of abnormal amounts of serum } \\
\text { rheumatoid factor by any method for which the result } \\
\text { has been positive in }<5 \% \text { of normal control subjects. }\end{array}$ \\
\hline (7) & Radiographic changes & $\begin{array}{l}\text { Radiographic changes typical of rheumatoid arthritis } \\
\text { on postero-anterior hand and wrist radiographs, which } \\
\text { must include erosions or unequivocal bony } \\
\text { decalcification localized in or most marked adjacent to } \\
\text { the involved joints (osteoarthritis changes alone do not } \\
\text { qualify). }\end{array}$ \\
\hline
\end{tabular}

\subsection{Treatment \& Therapy Options}

A cure for RA has been elusive; however there has been an improvement in treatment and therapy option over the last 50 years. Treatment options have evolved with improving medical advances, and have been able to successfully reduce the rate of mortality due to RA. Though a number of agents have proved useful in treating the disease, individual response to treatment is variable (Marra et al, 2005). Due to the aggressive nature of RA, it usually requires a lifelong treatment plan, which includes but is not limited to various intensive options, such as, medications, physical therapy, education, and in severe cases surgical intervention to relieve the 
symptoms and progressiveness of the disease. If diagnosed in the early onset of the disease, aggressive treatment options can delay and effectively manage further joint destruction. In addition to rest, strengthening exercises, and anti-inflammatory drugs, the current standard of care is to begin aggressive therapy with disease-modifying anti-rheumatic drugs (DMARDs), once the diagnosis is confirmed.

DMARDs, commonly used to initiate therapy, include methotrexate, hydroxychloroquine, and sulfasalazine. Minocycline may be as effective as or more effective than hydroxychloroquine. Methotrexate is the most commonly used initial DMARD agent. Increasingly, combination therapy is being used for added effectiveness. The idea of "triple therapy," using methotrexate, hydroxychloroquine, and sulfasalazine together, has been found to be more effective than any one of the drugs alone. Combining methotrexate with a variety of other individual drugs may add benefit as well.

\subsection{Thesis Objectives}

The primary objective for this thesis was to combine and incorporate statistical datasets collected by a previous student into a classifier. The method selected was a decision tree, to help classify the datasets of temperature measurements of two groups: patients and control subjects, in order to distinguish between the two groups. After that has been satisfied, we wish to be able to determine the level of severity of the illness in each patient and also determine the varying degree of severity in each specified joint. Even though RA is a bilateral disease and affects both sides of the body, the degree of joint degeneration varies from joint to joint. For example, the smaller joints of the hands and feet are where RA first manifests itself in the early onset of the disease before progressing to larger joints in the knees, elbows, ankles and neck regions. Supervised learning helps to make the task of classifying images of patients easier, because it 
takes an input variable (prior know parametric values) and from those variables predicts an output variable (e.g. presence or absence of RA). The goal was to find a function that best discriminates between the patients and the participants, and help eliminate factors that are not necessary in the assessment of RA. Future work would develop a prototype that is fully automated in order to assist physicians in diagnosing and assessing the disease status of their RA patients. Decision-tree learning is a common classification method (Ishii et al, 2010); it combines several binary tests in a tree like structure. The goal is to create a simple tree with a good predictive performance on the learning sample (Geurts et al, 2009). There are four specific objectives to be completed in this thesis:

1. The first objective was to complete statistical analysis of the various joints (Feet, Ankles and Elbows) for which data were collected but not analyzed (Adéa, 2009,Frize et al. 2009, Frize et al. 2010). From that earlier work, the scope of the analysis was limited to the joints of the knees and the joints in the hand of 13 patients (patient 1 through 13) and 18 control subjects (participants 1 through 18); it was established in that work that the knees, $2^{\text {nd }}$ and $3^{\text {rd }}$ MCPs (hand joints) were statistically good indicators of RA. So for this thesis, after the recruitment of additional patients and control subjects (13 patients and 10 control subjects), the first objective here was to complete statistical analysis on the remaining joints in the feet, ankles and elbows to determine whether among these joints, any one(s) could also be good indicators of RA and to also complete analysis of the knee and had joints of the patients and control subjects.

2. The second objective was to determine the presence of RA (discriminate between patients and control subjects). Using the various statistical dataset series (Min and Max temp, Min/Max and Median/Max, Mode/Min and Median/Min, Mode/Min and Mean/Min, 
Mode/Max and Median/Max, Mode/Max and Mean/Max, Min/Max and Median/Min, Min/Max and Mean/Min, Min/Max and Mean/Min, Median/Max and Mode/Min, Mean/Max and Min/Max, Min/Max and Mode/Min and Mode/Min and Median/Max) to discriminate between the patient and control subject groups. These datasets were inputted into the decision tree to determine which of the datasets generated a classifier that could discriminate between the patient and control subject groups. To create this classifier, the dataset of all the joints (knee, $2^{\text {nd }} \mathrm{MCP}, 3^{\text {rd }} \mathrm{MCP}$, and ankle) that statistically discriminated successfully between the patient and control subject groups were inputted all together in the decision tree.

3. The third objective was to determine which joints were more affected than others, because the progress of the disease varies from joint to joint depending on individuals. Even though the early onset of the disease manifests itself in smaller joints and progresses to larger joints, the rate of progress is not dependent on where the disease manifested itself; for example, the joint in the knee may deteriorate faster than those in the hands and might need more focused therapy option or surgical intervention than the joints in the hands even though the disease first manifests itself universally in the joints in the hands. This determination is necessary and important in order to help target certain joints in terms of a diagnosis, because each person's degree of severity in certain joint varies from one another. This was done by a process of elimination of variables (joints), where a combination of joints were inputted into the decision tree to determine which combination of joints generated the best classifier, indicating which joints were the best indicators of RA; and then the joints were further separated individually to determine which specific joints could on their own generate an excellent classifier. The results of the various dataset series were also compared to determine which combination helped improve the classification in terms of sensitivity and specificity. 
4. The fourth objective, after successfully separating the patient and control groups efficiently, was to determine the level of RA activity in the patient groups into LOW, MEDIUM and HIGH, using the same dataset series used to satisfy the previous objectives. From the patient group, the individual patients are at varying stages of the disease; in some patients the disease is at an early onset and in others the disease is in its advanced stages with visible deformities, and in others the disease is at an intermediate stage. The goal here was to generate a classifier that could classify these patients into these three groups. It is important to note that the classifier's discriminatory value in separating this patient group was established in terms of its specificity and sensitivity and the area under the Receiver operator characteristic curve as well as compared to the qualitative assessment of the rheumatologist.

\subsection{Thesis Outline}

Chapter 2 provides a technical literature review of the science behind the technology that is used in this thesis. It gives a medical evaluation of the patients by the Rheumatologist and their current disease status, and then it discusses the science behind thermal imaging - Stephan Boltzmann law, Planck's law \& electromagnetism. The chapter also looks at the some of the other clinical applications of Infrared Thermography. And lastly, the chapter looks at classification techniques, decision trees and some of its performance measures.

Chapter 3 discusses the methodology. It takes a look, briefly, at the ethical review \& clearance process, the recruitment of patients \& participants for this project, and also the data collection process. Then the chapter concludes with a discussion on the classification of images and the testing using Decision Trees. 
Chapter 4 begins with an analysis of the thermal images, and then discusses the resulting statistical data. It then shows the classification of the thermal images using decision trees and compares the best dataset series that help improve classification. The chapter concludes with a discussion of the results and discussion of whether the thesis objectives were met or not.

Chapter 5 provides the conclusion of this works and contributions to new knowledge, to the previous works done. It also proposes some new research objectives for future works in this study. 


\section{CHAPTER TWO}

\section{LITERATURE REVIEW}

This chapter provides a medical evaluation of patient joints (the hands, elbows, ankles, knees and feet) by a rheumatologist, from previous work (Adéa, 2009), to give a better understanding of the level of illness severity, as this differs from patient to patient. It also gives a brief history and it also delves into some of the past clinical applications of Infrared Imaging. And then the chapter looks at classification techniques, decision trees and some of its performance measures.

\subsection{Medical Evaluation of Patient joints}

This medical evaluation was necessary in order to determine the level of severity in each patient and determine the joints that were most affected by RA, because each patient has particular joints that are more affected than others. In order to complete this session, the patients were asked to fill out a questionnaire (Appendix A) asking them a brief medical history of their RA and also of any other joint issues that are not RA specific that could also be attributed to inflammation in the joints or that could cause an increase in temperature. The purpose of this is to eliminate any other factors that could contribute to false temperature readings or an increase in the temperature, because so much of this work is based on extracting as accurate as possible temperature measurements from the specified joints. The questionnaire contained questions about their age, number of years with the disease and also a ranking of the disease on a scale of 1 to 10 , where on the ranking scale 1 to 4 is LOW, 4 to 7 is MEDIUM, and 7 to 10 is $\mathrm{HIGH}$, to 
determine from the patient's perspective a disease level in terms of pain during inflammation. This medical evaluation provided us with pertinent information to help satisfy the fourth objective of this thesis. An assessment was also done by the rheumatologist (Appendix F)

\subsection{Infrared Imaging}

Infrared imaging is a technique that uses a thermographic camera to capture invisible infrared images; those images are converted to normal images that are visible to the human eye. For over five decades now, this imaging technique has had vast applications in many fields ranging from the military, to veterinary, to electronic maintenance applications, to food inspections. Thermography follows two principles: the mathematical model of Planck`s law which states that any object whose temperature is above absolute zero Kelvin emits radiation at a specific rate and with a distribution of wavelengths. This wavelength distribution is dependent on the temperature of the object and its spectral emissivity, $\varepsilon(\lambda)$. The spectral emissivity, which may also be considered as the radiation efficiency at a given wavelength, is in turn characterized by the radiation emission efficiency based on whether the body is a blackbody, grey body or selective radiators. The blackbody is an ideal body. It is a perfect absorber that absorbs all incident radiation and is conversely a perfect radiator, this implies that a blackbody absorbs and emits energy of the maximum theoretically possible at a given temperature $(\mathrm{Ng}, 2009)$ and the Stephan-Boltzmann law, which defines the relation between radiated energy and temperature by stating that the total radiation emitted by an object is directly proportional to the object's area and emissivity and the fourth power of its absolute temperature. Due to the emissivity of human skin being extremely high (within $1 \%$ of that of a black body), measurements of infrared radiation emitted by the skin can be converted directly into accurate temperature values. This makes infrared imaging an ideal procedure to evaluate surface temperatures of the body (Amalu, 
2003).The underlying idea of thermography is based on temperature changes in the human skin (see Appendix $\mathrm{G}$ for further details).

\subsection{Past Clinical Applications}

The use of infrared imaging as a medical diagnostic tool started in the early 1960's. The father of thermography as a screening technique was Lawson, who discovered in 1956 that the skin temperature over a cancer in the breast was higher than that of normal tissue. He also showed that the venous blood draining the cancer is often warmer than its arterial supply (Amalu et al., 2006). After this ground breaking discovery, there were other published works from other researchers such as, Lloyd-Williams et al., Gershon-Cohen et al., Swearingen and Connell et al. all confirming Lawson's findings on thermography as a clinical screening method (Dodd et al., 1969). However, the fall of thermography came in the 1970's after the sensitivity and specificity of infrared imaging was being questioned; its physical limitations (size, weight and cooling requirement), subjectivity in thermographic interpretation, and the expensive cost of IR systems were other factors.

But since the early discoveries of Lawson, and vast improvements made to the thermal IR cameras, there has been renewed interest in its medical application. These improvements include, the development of IR cameras with focal-plane arrays not requiring cooling, new detector materials with improved thermal sensitivity, and the development of smart image processing algorithms to enhance the interpretation of thermal signatures. The application of thermal infrared (TIR) imaging in medicine ranges from breast cancer screening, as earlier stated, to open heart surgery to image processing (Diakides, 2000). 


\subsection{Application of TIR imaging in $R A$}

The application of TIR imaging from past research has been limited to its use as an assessment tool (Collins 1974 and Ring 1974) and effective evaluation tool (Bird et al. 1979) in its application in RA. It has never been explored as a potential stand-alone diagnostic tool until now. In their research, Collins and Ring used a Thermal Index (TI) calculation based on the isothermal temperature difference to quantify the measurement of synovitis.

Thermal Index $=\frac{\sum(\Delta t \times a)}{A}$

where $\Delta t$ is the difference between the measured isothermal temperature and the lowest common isotherm recorded, $a$ is the area occupied by isotherm $\left(\mathrm{cm}^{2}\right)$ and $A$ is the total area of the thermogram $\left(\mathrm{cm}^{2}\right)$.

This TI reflected the mean temperature of a specified ROI. They established that TIR images of the hands, feet and knees can be used to identify patients with RA and as an assessment tool for the disease. In their work there was a decrease in the TI with treatment and therapy, and an increase in TI during periods of flare up. Their findings were confirmed by the research of Bird et al., where they also showed that "thermography can be used to assess inflammation of the synovial membrane and to evaluate the effectiveness of an RA treatment" (Bird et al., 1979).

However, in 1983, a new method of interpreting the thermal properties of TIR images of RA was suggested in the work of Salisbury to detect RA abnormalities in the knee, wrist, elbow and ankle joints (Salisbury 1983). He demonstrated that the quantification of synovitis by TIR imaging should be based on dysfunctional thermal patterns rather than by indexes, such as the 
TI, that are based on absolute skin temperature values. However, it has been shown that, various external factors can affect the skin's surface temperature such as hot/cold beverages consumption, emotional state, circadian variation, physical activity, environment temperature and humidity, etc. (Reinberg 1976). Salisbury et al. introduced a Heat Distribution Index (HDI) that show the pattern and spread of the temperature distribution over the surface of a joint. They went on to prove that the HDI correlated better with joint inflammation and synovitis than Collins and Ring's TI. The TI proved to be less significant in identifying the presence of inflammation and was even less significant in identifying inflammation in afternoon TIR readings. The TI is more variable and less reproducible than the HDI, since it is greatly affected by the patient's circadian rhythm and the environment in which the images are taken. The HDI was able to identify a normal thermal distribution in the absence of inflammation in the knee, ankle, wrist and elbow joints. Alternatively, inflammation of the synovial membrane was repeatedly detected by the HDI analysis and these results were not affected by the time of day of the TIR readings (Adéa, 2009).

Salisbury et al.'s HDI is calculated based on the relative frequency distribution of the thermogram via a 256 grey level TIR image. A ROI of interest is delimited to cover the joint to be analysed. A pixel count is made at each grey level within the ROI and each resulting number is divided by the number of pixels in the ROI. Normal joints present a narrower relative frequency distribution (lower standard deviation) than a diseased joint. Salisbury et al. also added that the distribution of heat over a normal joint results in a positive temperature gradient from the centre to the periphery of the joint. They also noted that normal joints do not have localized areas of increased temperature, hot spots. An abnormal joint with synovitis can 
therefore be described as a joint presenting with a negative thermal gradient with localized hot spots within the joint's ROI; this was also confirmed by Adéa in the earlier research.

In this thesis, TIR image analysis of the joints, the hands, elbows, feet, ankles and knees were all based on the temperature distribution methodology introduced by Salisbury, supported by many others and implemented by Adéa and myself. The temperature distribution pattern was analysed to see if abnormal localized regions of increased or decreased temperatures can be used to classify participants with RA.

\subsection{Pattern Classification techniques}

Pattern classification is the method of exploring the model that generated the patterns explored. Depending on different information over the model or the exploratory method used, pattern classification techniques can be further organized in different taxonomies. An example of such taxonomy can be seen in a model of statistical versus syntactical, parametric versus nonparametric dichotomies. For the statistical technique, the focus is on statistical properties of patterns, as was done in earlier work. Whereas a syntactical (or structural) like a decision tree uses models with some logical rules, as was done in this thesis. On the other hand, classconditional densities of the model are assumed to be known in parametric techniques and not known in nonparametric ones. Examples of other taxonomies are deterministic versus stochastic (randomness is present during model search or learning for the latter), supervised versus unsupervised (learning is guided through target labels in the former), and metric versus nonmetric (relative to feature values) (Unay 2006). 


\subsubsection{Decision Tree}

In the medical field, there are often times situations, where the medical decision maker will be faced with a sequential decision problem involving decisions that lead to different outcomes depending on chance. If the decision process involves many sequential decisions, then the decision problem becomes difficult to visualize and to implement. Decision trees are indispensable graphical tools in such settings. They allow for intuitive understanding of the problem and can aid in decision making (Ishwaran and Rao).

A decision tree is a graphical model describing decisions and their possible outcomes. Decision trees consist of three types of nodes (figure 2-3)

1. Decision node: This node often represents the decisions that can be made, and shows all the distinct options available at a node.

2. Chance node: This node shows chance outcomes. These are events that can occur but are outside the ability of the decision maker to control.

3. Terminal node: These are end nodes, where there are no further decision nodes or chance nodes. These nodes depict the final outcomes of the decision making process.

In this thesis, Rulequest's See5 software was used to generate the classifier used as a tool in the decision making process of the diagnosis of RA (1) determine the presence of RA from two distinct groups; (2) determine the joints that best indicate the presence of RA; and (3) determine the level of severity in the presence of RA. 


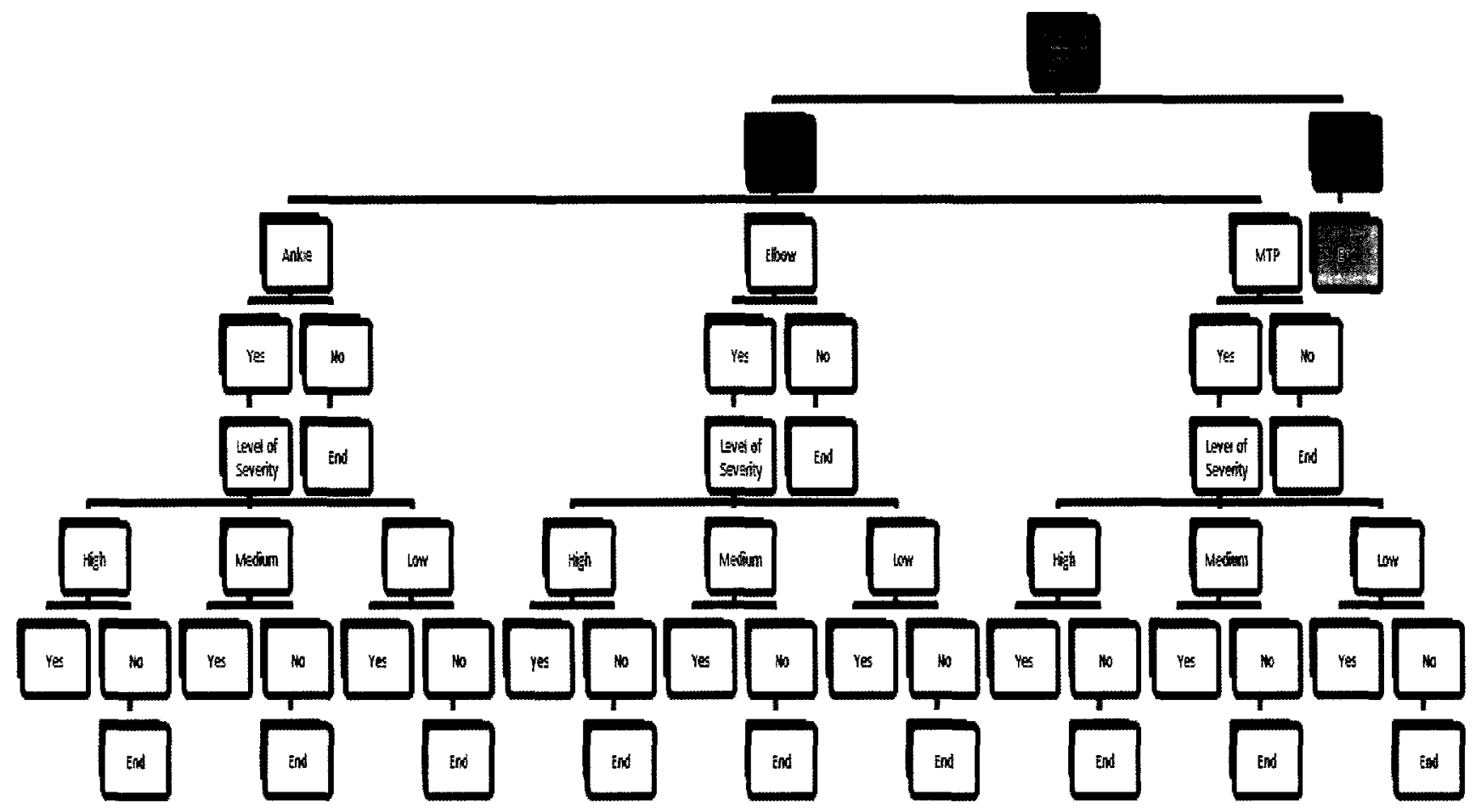

Fig. 2-1: Preliminary sample model of the proposed decision tree for $\mathrm{RA}$ diagnosis.

\subsubsection{Bootstrapping}

Bootstrapping in its simplest form, simply means, instead of repeatedly analyzing subsets of the data, you repeatedly analyze subsamples of the data. Where each subsample, is a random sample with replacement from the full sample. Depending on the needs of the user, this method can be implemented on anywhere from 50 to 2000 subsamples. However, there are also various sophisticated bootstrap methods that can be used not only for estimating generalization error but also for estimating confidence bounds for network outputs (Efron and Tibshirani 1993).

\subsubsection{Ensemble Decision Tree Classifier}

An ensemble decision tree classifier is simply the creation of numerous decision trees for classification purposes. This is necessary because, single trees are known to be fairly unstable 
especially with smaller databases; since the designing of a tree depends greatly on the dataset and variables used for training and testing. Therefore, relying on the creation and results of only one tree is inadequate. To avoid the instability of using single trees and also maintain robustness in the performance of the decision tree (DT) classifier, an ensemble method was used. The ensemble classifiers were created by bootstrapping the original training sets and allocating these into bootstrapped training subsamples (bTRAIN). In this method, the DT classifier created a tree from each training subset and tested each tree on the test set. A typical tree is created using the best split among all variables; however, another issue with DTs is that they choose variables according to local optima. This does not necessarily guarantee a global optimum (Mitra and Acharya, 2003). Once a tree is created in See5, the variables are ranked according to usage where some variables are not used at all. In order to optimize the DT classification once an initial tree was created, a variable used in the split was removed, and a new tree was created from the remaining variables. The performance of the new tree was monitored to observe the changes caused by the removal of the variable, if the removal had an adverse effect on the performance of the tree, then the removed variable was re-introduced back and another variable removed to create a new tree. These steps were repeated until the best performing tree was found. This tree was then used as one of the trees of the ensemble classifier. This same process was repeated for each bootstrapped training subset in the DT classifier.

\subsection{Performance measures of Classification Techniques}

\subsubsection{Confusion Matrix (Contingency Table)}

The confusion matrix is a matrix that displays the number of scores that are correctly predicted and incorrectly predicted by the model. The figure below shows the confusion datasets 
for a two-class problem. Although it is worth noting that a confusion matrix can be extended to an n-class problem (Tan et al., 2006).

Table 2-1: A 2 x 2 Contingency Table

\begin{tabular}{|c|c|c|}
\hline & Positives & Negatives \\
\hline 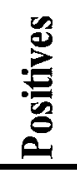 & $\mathrm{TP}$ & FP \\
\hline : & FN & TN \\
\hline
\end{tabular}

\subsubsection{Correct Classification Rate (CCR)}

This simply refers to the number of correctly predicted scores by the classifier. It is also known as the accuracy of the classifier:

$$
\mathrm{CCR}=\frac{\# \text { of correct prediction }}{\text { Total \# of prediction }}=\frac{T P+T N}{T P+T N+F P+F N}
$$

\subsubsection{Error Rate}

This simply refers to the number of incorrect predictions. The prediction model for the training data gives the apparent error which is usually lower than that of the true error found using the test data.

$$
\text { Error rate }=\frac{\# \text { of wrong predictions }}{\text { Total \# of predictions }}=\frac{F P+F N}{F P+F N+T N+T P}
$$




\subsubsection{Sensitivity}

The sensitivity, also known as, the true positive rate (TPR) of the classifier - given that the actual value is positive, is the fraction of value that is actually classified as true positive. In medical informatics such rates would be the positive result when the outcome is abnormal (Prince and Links, 2006).

$$
\mathrm{TPR}=\frac{T P}{T P+F N}
$$

\subsubsection{Specificity}

The specificity, also known as, the True Negative Rate (TNR) of the classifier - given that the actual value is negative, is the fraction of value classified as true negative. In medical informatics, such rates would be the negative result when the outcome is normal (Prince and Links, 2006).

$$
\mathrm{TNR}=\frac{T N}{T N+F P}
$$

Can also be expressed as,

$$
\text { TNR }=1-F P
$$

\subsubsection{Prevalence}

Prevalence is the prior probability that the subject has the disease before the test is applied; while the posterior probability is the probability of the disease after a test has been 
applied. Rare outcomes have low prior probability. In order to assess the usefulness of the test, a comparison between the prior and posterior probability can be made. Predictive values are used for medical diagnostics evaluation to determine the probability that the diagnosis is correct. This is highly dependent on the patient population that the test is used for (Altman and Bland, 1994).

\subsubsection{Positive Predictive Value}

The PPV is the probability of the actual disease (positive outcome) given that the prediction is positive. PPV is proportional to prevalence therefore if prevalence of disease is low, PPV will not be close to 1 (even with high specificity and sensitivity). A disease with low prevalence would have a sharp decrease in the PPV. The PPV can be calculated using the following equations (Kelly et al., 2008).

$$
\mathrm{PPV}=\frac{\text { Sensitivity } \times \text { Prevalence }}{\text { Sensitivity } \times \text { Prevalence }+(1-\text { Specificity }) \times(1-\text { Prevalence })}
$$

Can also be expressed as,

$$
P P V=\frac{T P}{T P+F P}
$$

\subsubsection{Negative Predictive Value}

The NPV is the probability of a non-disease or negative outcome given that the prediction is negative. A high NPV indicates a greater chance that a negative test has no abnormality. A rare outcome (low prevalence) would indicate a greater assurance that a patient with a negative test is correctly diagnosed (Altman and Bland, 1994). The NPV can be calculated using the following equations (Kelly et al., 2008): 


$$
\mathrm{NPV}=\frac{\text { Specificity } \times(1-\text { Prevalence })}{\text { Specificity } \times(1-\text { Prevalence })+(1-\text { Sensitivity }) \times \text { Prevalence }}
$$

Can also be expressed as,

$$
\mathrm{NPV}=\frac{T N}{T N+F N}
$$

\subsubsection{Receiver Operating Characteristic (ROC) Curves}

ROC curves provide a visual model that displays the trade-off between sensitivity and specificity. The ROC curve is produced by graphing the false positive rate (FPR) which is the same as "1-Specificity" against the true positive rate (TPR). The visual model, in figure 2-4 presents points that are of importance as noted by Fawcett (2006).

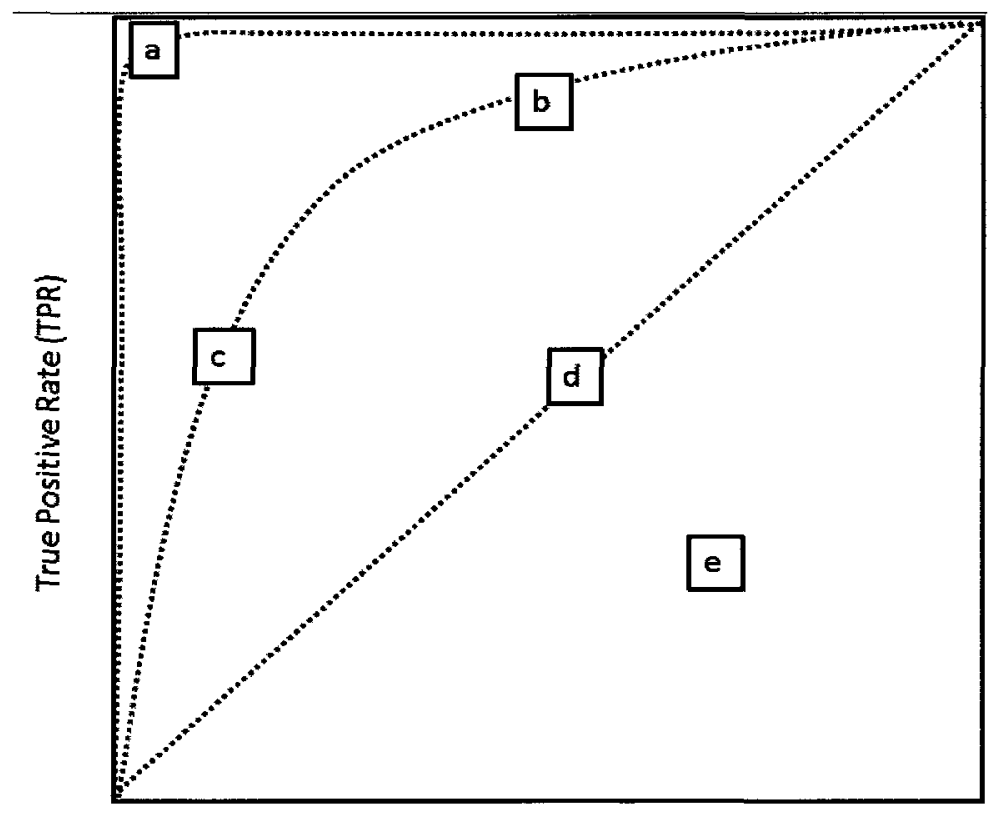

False Positive Rate (FPR)

Fig. 2-4: An ROC curve and the different points of significance (created from Fawcett 2006).

1. The coordinate $(0,0)$ is located where no positive classification is given therefore no false positive classification exists. The opposite point is $(1,1)$ where the classifier assigns a positive class label to all points, therefore the FPR is at a maximum. 
2. Point a is a point, where most points are correctly classified.

3. At point $\mathbf{b}$, the TPR is higher than a, but the FPR has increased as well.

4. Point $\mathrm{c}$ has a low FPR; however, its true positive rate is almost at $60 \%$.

5. The diagonal line, point $\mathbf{d}$, indicates random guessing. A point on the linear mark indicates that TPR and FPR are equal and the classifier performance is random. This classifier does not contain useful information.

6. The point $\mathbf{e}$ is below the random line and is worse than random guessing. Having a classifier performance in this area may indicate that useful information is contained in the classifier, but the application is incorrect.

Various operating points under different threshold values of the model provide the ROC

curve. Though the curve assesses the performance of the model at each individual point, the area under the curve (AUC) presents an assessment of the whole model. The AUC is a scalar value of the ROC curve and is used to compare the performance of different models (Yuu, 2010).

\subsubsection{Area Under the Curve}

The AUC of the diagonal line has a value of 0.5 while the AUC containing point a, has a value closer to 1 . Values less than 0.5 are worse than random guessing. The AUC is used to compare the performance between classifiers and will always have a value between 0 and 1 . The AUC contains statistical property as it is the probability that the "the classifier will rank a randomly chosen positive instance higher than a randomly chosen negative instance (Fawcett, 2006)." The AUC is a measurement of the model's effectiveness in discriminating outcomes as shown in the Table 2-2 (Ambrosius 2007). 
Table 2-2: AUC index and its effectiveness for discrimination

\begin{tabular}{rrl}
\multicolumn{1}{l}{ Min } & Max & Effectiveness \\
\hline \hline & $\leq 0.500$ & No discrimination (Random) \\
\hline 0.500 & $<0.700$ & Poor discrimination \\
\hline 0.700 & $<0.800$ & Acceptable discrimination \\
\hline 0.800 & $<0.900$ & Excellent discrimination \\
\hline 0.900 & 1.000 & Outstanding discrimination \\
\hline \hline
\end{tabular}




\section{CHAPTER THREE}

\section{METHODOLOGY}

This chapter discusses the ethical review and clearance process, the recruitment of patients and control subjects for this project, the protocol to which the participant (patients and control subjects) had to adhere to for participation in the research, and the data collection process. The chapter concludes with a discussion of the classification of the datasets using Decision-Trees.

\subsection{Ethics Review}

For this research, the original proposal (submitted by Adéa) was approved by the Ethics board at the three institutions involved in the research, The Ottawa Hospital Riverside Campus, Carleton University and The University of Ottawa. Initial ethical clearance was given by the three ethics boards under the title of "Severity of illness measures in rheumatoid arthritis from multi-spectral imaging". The principal investigator for the Ottawa Hospital for this research was Dr. Jacob Karsh, a rheumatologist from the Riverside Campus, where the patients were recruited. The two Principal investigators at Carleton University and University of Ottawa were professors from the Faculty of Engineering and Design at Carleton University and the School of Information Technology and Engineering at the University of Ottawa and students from the two institutions. The ethics clearance was requested from the Ottawa Hospital Research Ethics Board (OHREB), since the patients were recruited from the hospital. Also Ethics clearance was requested from the Carleton Research Ethics Committee and The University of Ottawa Research Ethics Board since the research involved the use of physical resources and equipment's at both institutions. This thesis is the second phase and continuation of the objectives outlined in the 
original proposal submitted, which was to explore the effectiveness of thermal Infrared imaging as a viable diagnostic tool to aid in the early detection and effective monitoring of RA. The continuation of this study has added valuable knowledge to Dr. Frize's Medical Informationtechnologies Research Group (MIRG) at Carleton University and has also raised other budding research questions that would aid in gearing the research towards the next phase.

\subsection{Recruitment of Patients}

Following the same recruitment protocol as was in the previous study (Adéa, 2009), the research participants were patients already diagnosed with RA from the Ottawa HospitalRiverside Campus aged 19 years old and above. These patients were recruited by their Rheumatologist, Dr. Jacob Karsh, who informed them of the ongoing project and if they were interested in participating in it. Since RA is a disease that affects both sexes, patients of both sexes and of any age from 19 and above were asked to participate in the study. Dr. Karsh provided the patients with information about the study. If the patients were interested in participating in the research, the doctor provided them with the participation package, which contained the information and consent forms, and providing further information about the study and the consent form to sign (Appendix B). Once they had read and understood the information letter and still wanted to be a part of the research study, they directly contacted the coinvestigator's student at Carleton University or the University of Ottawa to further discuss participation and to ask any further questions. After signing the consent form and discussing the expectation for the imaging session, an imaging session is scheduled. Those patients, who consented and completed the consent form, permitted the usage and storage of the digital IR images of their limbs and joints. No images of participants' faces were taken and no personal information was shared with Carleton University or the University of Ottawa's research teams. 
The only criteria for exclusion from the study was whether the patients were diagnosed with joint issues not attributed to RA, patients who had recently suffered any kind of trauma that affected their joints, or patients with joint sepsis. The patients who participated in the study were not given any research incentives to participate in the study, and were not coerced into participating neither was there any adverse effect to their quality of care.

The control group participants, like the patients recruited, followed the same protocol and were subjects aged 19 years and above from both University Campuses, and had not been diagnosed with RA, or had any joint issues or recent trauma's; they were generally in good health and without pain or symptoms resembling RA. These control subjects were recruited to establish a range of temperature distributions for normal subjects and to provide a baseline for what was to be identified as normal. There were no incentives to participate in the study or were there any coercion of the control participants. They were given the same participation package as the RA patients (see Appendix B) and signed the same consent form which permitted the usage and storage of the digital IR images of their limbs and joints. No images of control subjects' faces were taken and no personal information was shared with Carleton University or the University of Ottawa's research teams.

In the first study by Cynthia Adéa, thirteen patients diagnosed with RA were recruited from the Ottawa Hospital-Riverside Campus and eighteen control subjects were recruited from Carleton University, the University of Ottawa and a few from outside the campuses that participated in the original research study. However, while furthering the project, for the purposes of classification, it was necessary to recruit more patients and control subjects to help improve the classification, so an additional thirteen patients were recruited again from the Ottawa Hospital-Riverside Campus and ten additional control subjects were recruited. 


\subsection{Collection of Data}

\subsubsection{Infrared Camera Specification}

The FLIR A320 Researcher Infrared Camera from FLIR Systems was used to capture the TIR images. This IR camera uses an uncooled microbolometer focal plane array of $320 \times 240$ pixels at a refresh rate of $50 / 60 \mathrm{~Hz}$. It operates in the $7.5 \mu \mathrm{m}$ to $13 \mu \mathrm{m}$ of the far IR spectral range of the electromagnetic spectrum. The A320 has a $24 \mathrm{~mm}$ germanium lens with an anti-reflective coating. The germanium lens renders a field of view that is $25^{\circ} \times 18.8^{\circ}$ with a minimum focus distance of $0.3 \mathrm{~m}$. The camera's spatial resolution is $1.3 \mathrm{mrad}$, which means that at a distance of $1 \mathrm{~m}$ each pixel represents a square of $1.3 \mathrm{~mm} \times 1.3 \mathrm{~mm}$. The A320 has a thermal sensitivity of $0.07{ }^{\circ} \mathrm{C}$ at $30^{\circ} \mathrm{C}$ and can measure temperature ranges from $-20{ }^{\circ} \mathrm{C}$ to $+120{ }^{\circ} \mathrm{C}$. The camera was connected to a laptop via an Ethernet cable, thereby capturing sequences of 14 bit digital thermal images at a maximum speed of 60 frames per seconds (FLIR Systems 2007).

\subsubsection{Infrared Camera and recording settings}

The software used during the imaging sessions was the ThermaCAM Researcher Pro 2.9 from FLIR Systems. It was via this software that measurement corrections were inputted by the user, the emissivity, the camera distance, the ambient temperature and the relative humidity. The A320 radiometric camera outputted emissivity measurements to the ThermaCAM software which converted the measurements into absolute temperatures while taking into consideration all the inputted corrections. The camera settings used for this thesis were as follows. The emissivity was kept constant at 0.98 for the skin. The camera distance ranged from $0.5 \mathrm{~m}$ to $1 \mathrm{~m}$ and was manually adjusted for every image captured via the settings options of the software. The ambient temperature and relative humidity of the environment were measured for each session 
and were $23 \pm 1{ }^{\circ} \mathrm{C}$ and $40 \pm 2 \%$ respectively for all the sessions. The image intervals for the recording conditions were set at 2 , which means 30 images were taken per second during each recording time. The image presentation box was enabled to allow the user to view the images live and make manual adjustments to the camera for the best image captured (Adéa, 2009).

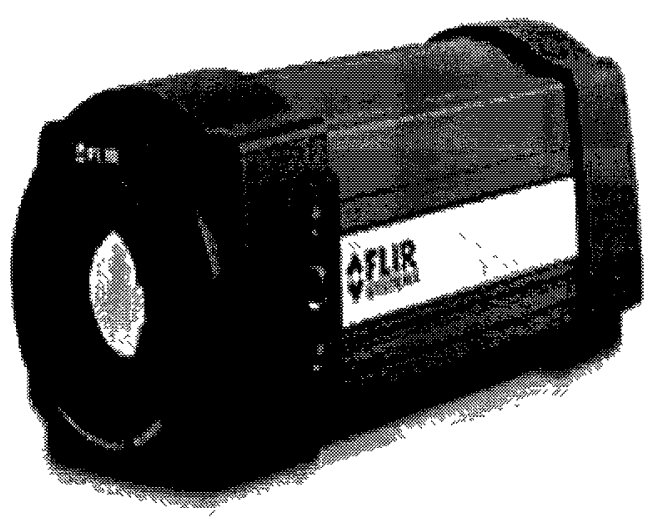

Fig. 3-1: A320 Infrared Camera from FLIR Systems (FLIR Systems, 2007)

Images were recorded in *.seq format for sequences of images and were subsequently exported into Matlab as *.mat files. The *.mat files contained the temperatures of each pixel of the image and information about the environmental settings.

\subsubsection{Medical Thermal Infrared Imaging Protocol}

Prior to the imaging session, each participant was given these guidelines to follow (adapted from Herry, 2007):

- Do not consume alcoholic beverages twenty-four hours prior to the session.

- Do not consume hot beverages at least one hour prior to the session.

- Do not wear rings, necklaces, anklets and/or bracelets during the session. 
- Do not use talcum powder, lotion, drug or deodorant on the skin on the day of the session.

- Avoid prolonged sun exposure at least one week prior to the session.

- Avoid smoking at least two hours prior to the session.

- Avoid any intense physical exercise at least four hours prior to the session.

- Avoid procedures such as electromyography, acupuncture, myelography, transcutaneous electrical stimulation, hot or cold patches, or any other form of physiotherapy at least twenty-four hours prior to the session.

\subsubsection{Recording Procedures}

There were certain protocols that needed to be followed for the room to be conducive for an IR thermographic session. These sessions took place in a room that had no windows to avoid any reflective effects of light captured by the camera. There were no shiny or plastic floors and the only lights used were fluorescent lights. The temperature and humidity were measured before and after every session. They were uniform throughout the room and were kept constant throughout the entire session. The patients and control subjects were asked to adhere strictly to the medical thermal infrared protocol prior to the session. They were also asked to wear or bring along a sleeveless shirt and shorts to the session and to remove their socks and shoes to facilitate the imaging of different views of the feet and ankles (Adéa, 2009).

Before the recording session, the participants (patients and control subjects) were seated in a chair with their arms resting on the chair arms to the side with no contact to their body's and their bare feet relaxed on the floor for a cooling down period of 15 minutes to adjust their body temperature to reflect the ambient room temperature. During this time, the participants' surface body temperature was cooled sufficiently in a room remaining constant around $23{ }^{\circ} \mathrm{C}$. The participants remained seated without any body parts in contact with one another and without 
removing their feet from the bench to ensure that the surface temperature of their skin correctly reflected the internal temperature of the joints and limbs.

During the imaging session, the joints of interest were the elbows, wrist and hand joints, knees, ankles and feet joints. Each joint was captured from different anatomical views.

For the elbows, the first image was of the extended elbow and the images captured were of the posterior and anterior view of the frontal anatomical plane. Then the participants (patients and controls) were asked to flex their elbows at 90 degrees and images captured in the frontal plane. The flexed elbows were also imaged in the right and left sides. The images of the different anatomical views are shown below in grey level TIR images of the images that were captured of the elbows.

Table 3-1: Grey Level Thermal Infrared Views of the Elbow (Adéa, 2009)

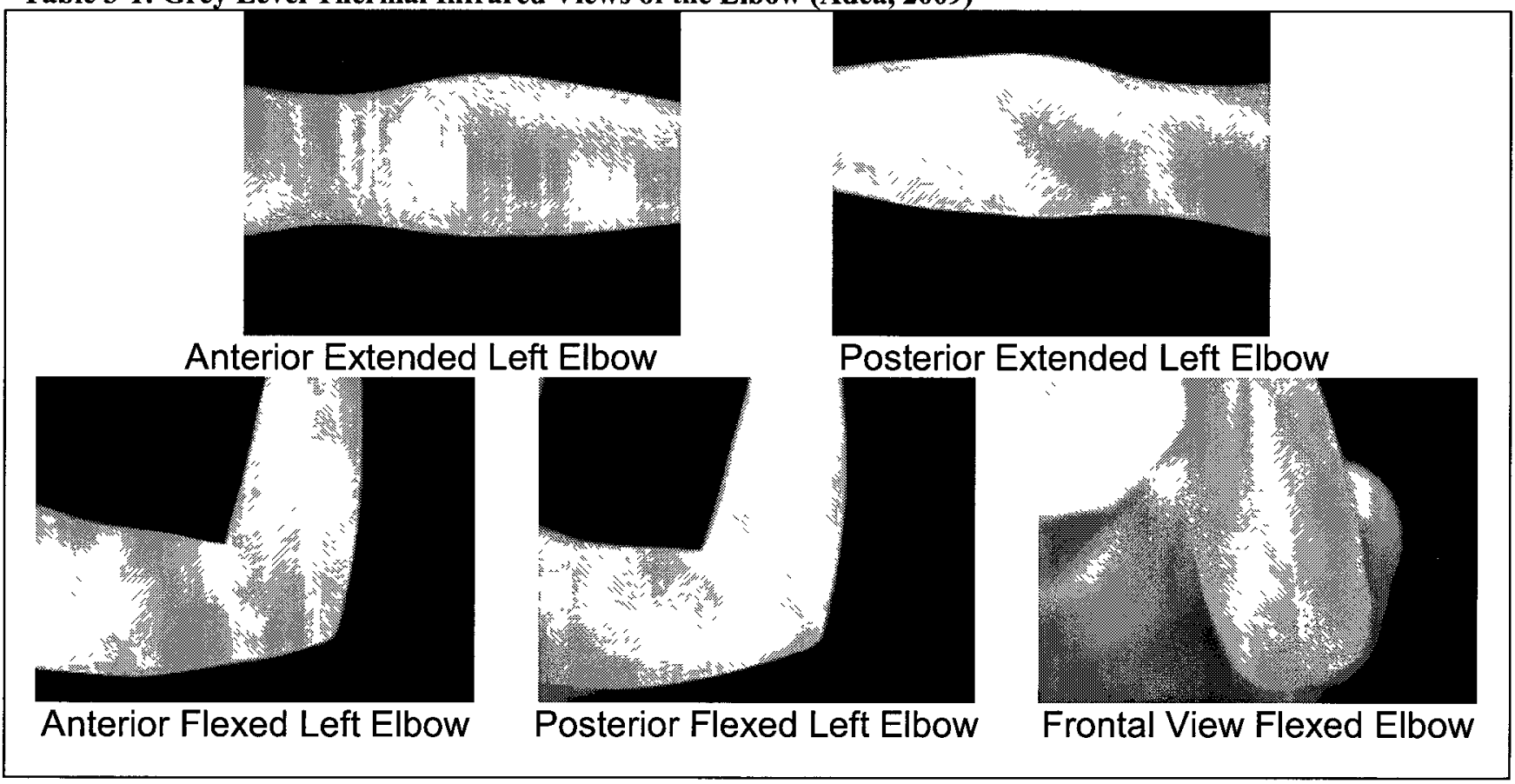

For the wrist and hand joints, wrists and palm were extended and the joints were imaged in a single capture in the posterior and anterior view of the frontal anatomical plane. The images 
of the different anatomical views are shown below in grey level of TIR images that were captured of the wrist and hand joints.

Table 3-2: Grey Level Thermal Infrared Views of the Wrist and Hand (Adéa, 2009)

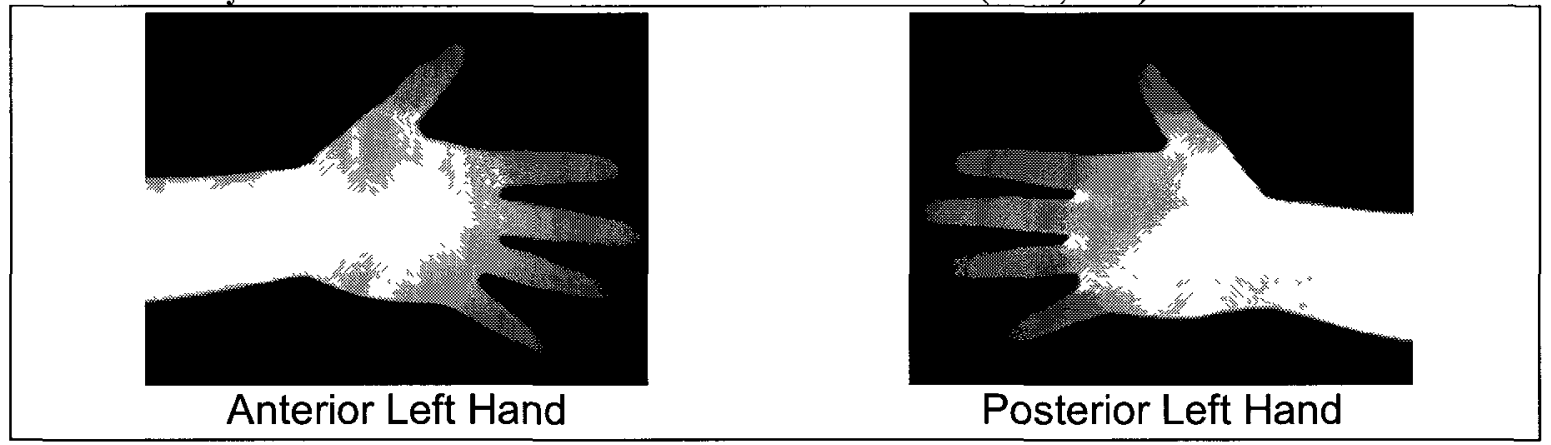

The images of the knees were first from an extended straight standing up position and were captured in the posterior and anterior view of the frontal anatomical plane. The images of both extended knees were captured at the same time and then that of individual extended knees separately taken also. Also images were captured while the participants were in the sitting position (knees flexed at 90 degrees) in the anterior frontal view. The images of both flexed knees were captured at the same time and then that of individual flexed knees separately captured. The individual's flexed knees were also imaged in the right and left sides. The images of the different anatomical views of the knees are shown below in grey level TIR images. 
Table 3-3: Grey Level Thermal Infrared Views of the Knee (Adéa, 2009)

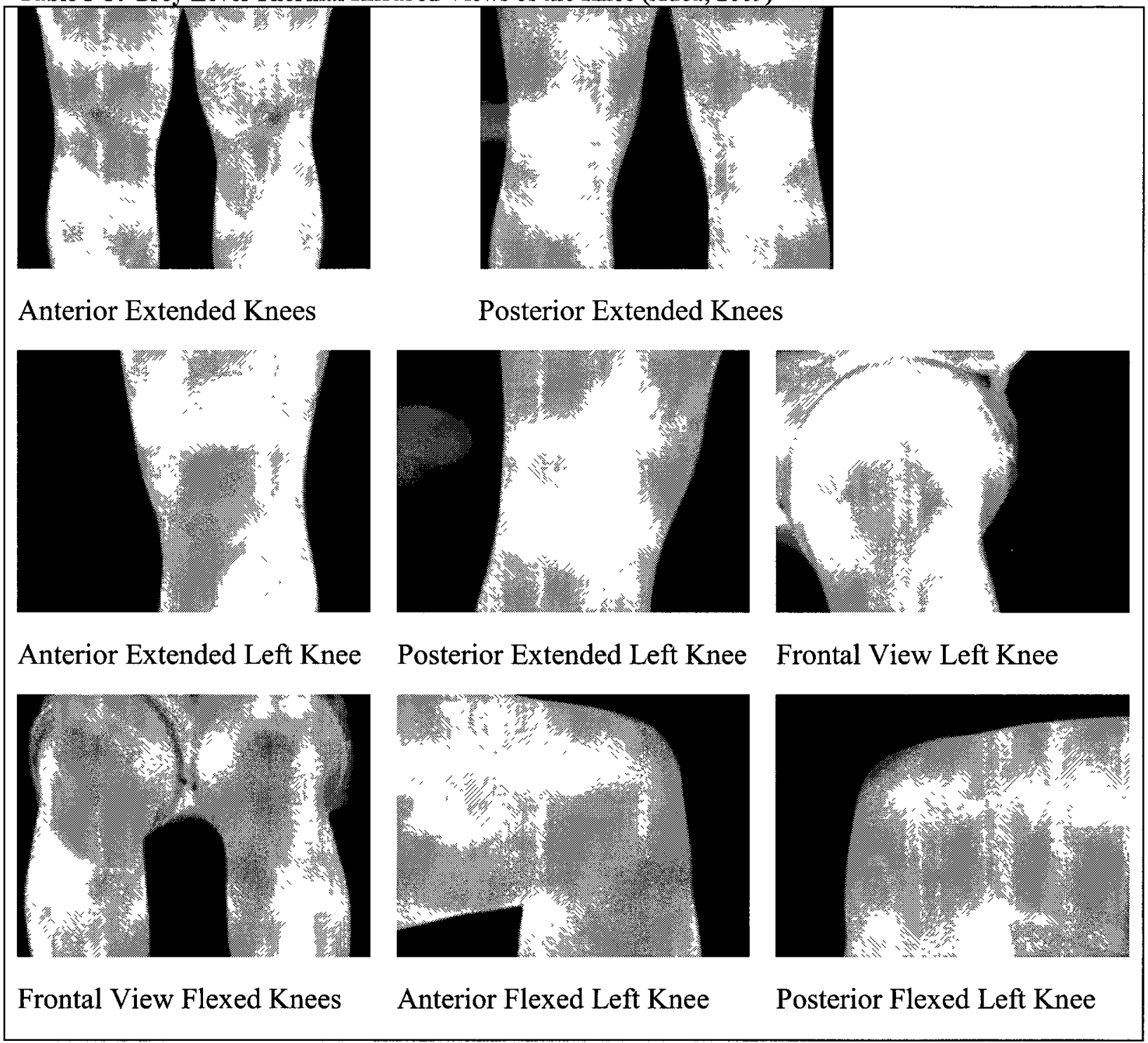

For the ankles and feet joints, the images were captured in the posterior view of the frontal anatomical plane. For the posterior view, images were captured of both ankles at the same time and then that of individual ankles separately. Also, images were captured in the right and left sides of the ankles and feet joints. The top and the bottom view of the feet were also captured. For the top of the feet, images were captured at two distances $-0.5 \mathrm{~m}$ and $1 \mathrm{~m}$ respectively. The top views were captured of both feet at the same time and then that of individual feet separately. The bottoms of the feet were captured at the end of the session in two 
rounds. The first round was captured immediately after the imaging session and the second round was captured after a 5 minute resting and cooling period. During this cooling period, the feet remained propped up and relaxed on a stool to allow the skin surface of the feet to efficiently represent the intra-articular temperature of all the feet joints (Adéa, 2009). The images of the different anatomical views of the ankles and feet are shown below in grey level TIR images.

Table 3-4: Grey Level Thermal Infrared Views of the Feet (Adea, 2009)

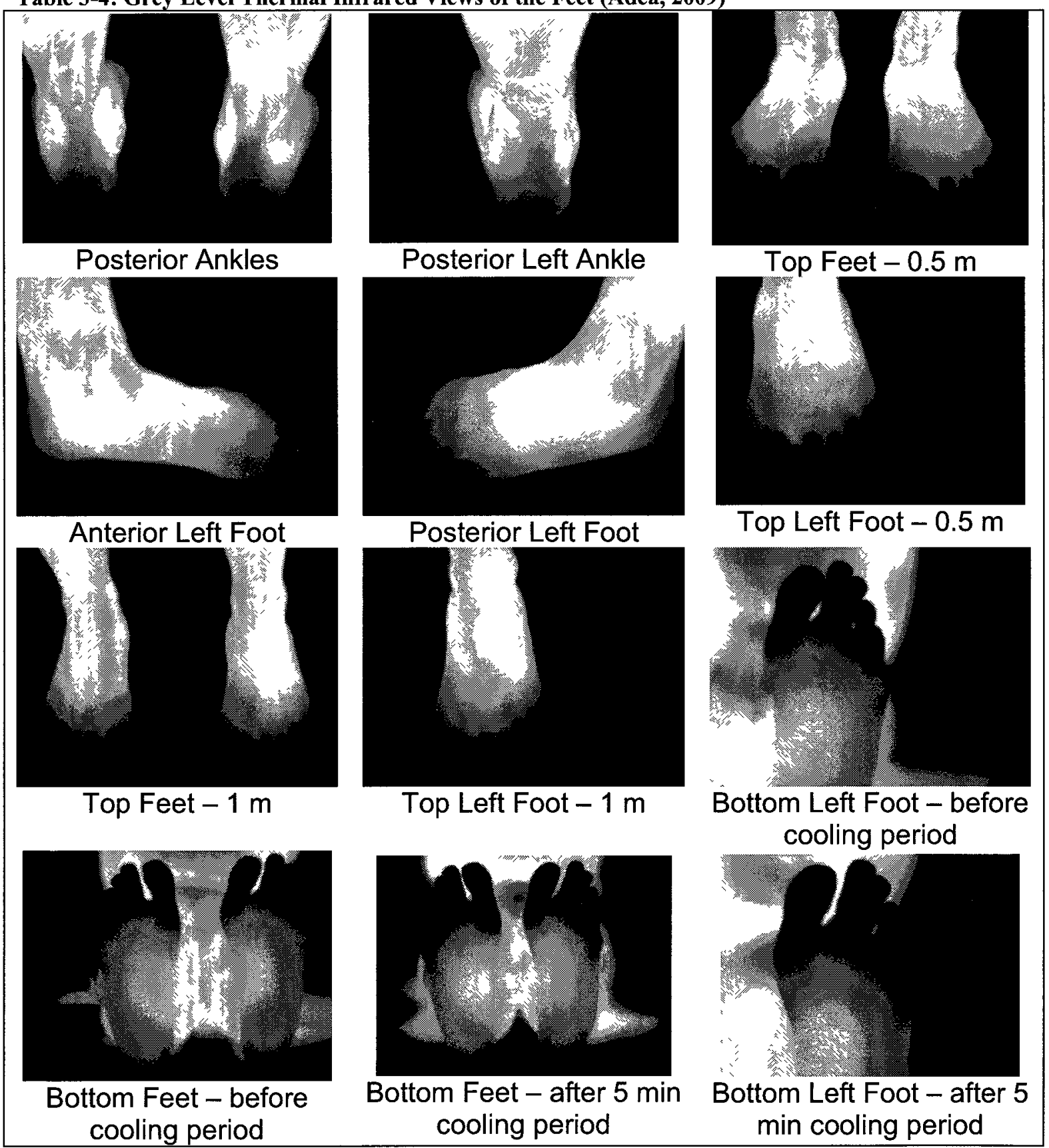




\subsection{Data Analysis}

In order to be able to process the TIR images and perform the necessary statistical tests and subsequently classify the images, it is important to first review the aforementioned objectives of the thesis. The first objective was to complete statistical analysis on the remaining joints (elbows, ankles and feet) that were not analyzed in earlier work by Adéa which was limited to the analysis of the joints in the hands and knees, to determine if other joints could discriminate statistically between the patients and control subjects datasets. The second objective, after establishing the joints that could statistically discriminated between the patients and control subjects, was to use these temperature measurements as inputs into a decision tree to discriminate between the patients and control subjects. The third objective was to use these statistical datasets in a decision tree to determine (1) the combination of joints that could generate a classifier that determined the joints that were good indicators of RA, (2) the specific joints of the four joints (knee, $2^{\text {nd }} \mathrm{MCP}, 3^{\text {rd }} \mathrm{MCP}$ and ankle) that best indicated the presence of RA and also compared the various datasets series to determine which series combination generated the best classifier with the highest specificity and sensitivity. The fourth and last objective was to determine the level of RA activity in the patient groups and separate them into three group classes: LOW, MEDIUM and HIGH, using the same dataset series that successfully satisfied the second objective and then the results from the classification were compared with the rheumatologist's evaluation of RA activity in those patients. 


\subsubsection{Conversion of TIR images to Grey Scale}

The first step to accomplishing the objectives specified in this thesis, was the conversion of the *.seq FLIR file into a *.mat MATLAB file using the FLIR software (ThermaCAM Researcher Pro 2.9). The images, now a matrix of temperatures in Kelvin, using the mat2gray function in MATLAB were converted into a normalized matrix ranging from 0 to 1 . Then these normalized matrix were displayed via MATLAB's imshow function, displaying a gray scale image. The reason for this transformation to gray scale was in an effort to eliminate any biases that a pseudo-colour image may create when the image was being reviewed subjectively (by the researcher). This method, is corroborated by past research that has shown that pseudo-colours when interpreting TIR images is not a good practice given that humans are subject to psychological effect of hot and cold colours (Anbar 1991).

The next step after converting these images to gray scale was selecting the region of interest to be analyzed. In this thesis, the Regions of Interests (ROIs) were manually selected from the gray scale images using anatomical reference points with the aid of MATLAB's roipoly function. The selection of the region of interest played a significant role in the analysis of the images. However there were some drawbacks to this method because of its susceptibility to human error; there is no standard method of selection, which leaves the selection of the ROI to the interpreter's discretion. Therefore it was important to carry out the selection process with as much accuracy as possible. This meant that for each joint, the anatomical reference points were determined by determining the location of the synovial membrane (Moore, 2007). This determination was made through careful assessment and studying of the various anatomical reference point of each joint analysed. The rheumatologist in this study gave an assessment of where this reference points were located in the joints, however was unavailable to review the 
images; this determination was also made from studying literature on where this reference points are located in the joint. The determination of the ROI was selected using a combination of this two methods. The joints analysed were those in the hands, the elbows, the feet, the ankles and the knees. For instance, in the joint of the knee, anatomical reference point used in the selection of the region of interest was the patella. The patella was used as the main anatomical reference point, because the synovial joint is found underneath it. The anatomical reference points used for the MCP, PIP and MTP joints are the articulations of these joints themselves. The MCP and PIP joints were identified by their ovoid surface. The synovial joint is found directly under the ovoid surface. The images shown below show the gray scale datasets of the original TIR image with the manually selected ROIs.

Table 3-5: Regions of Interests Manually Selected for the 2nd and 3rd MCPS, the 2nd and 3rd PIPs, the Wrist and the Knee (Adéa, 2009)
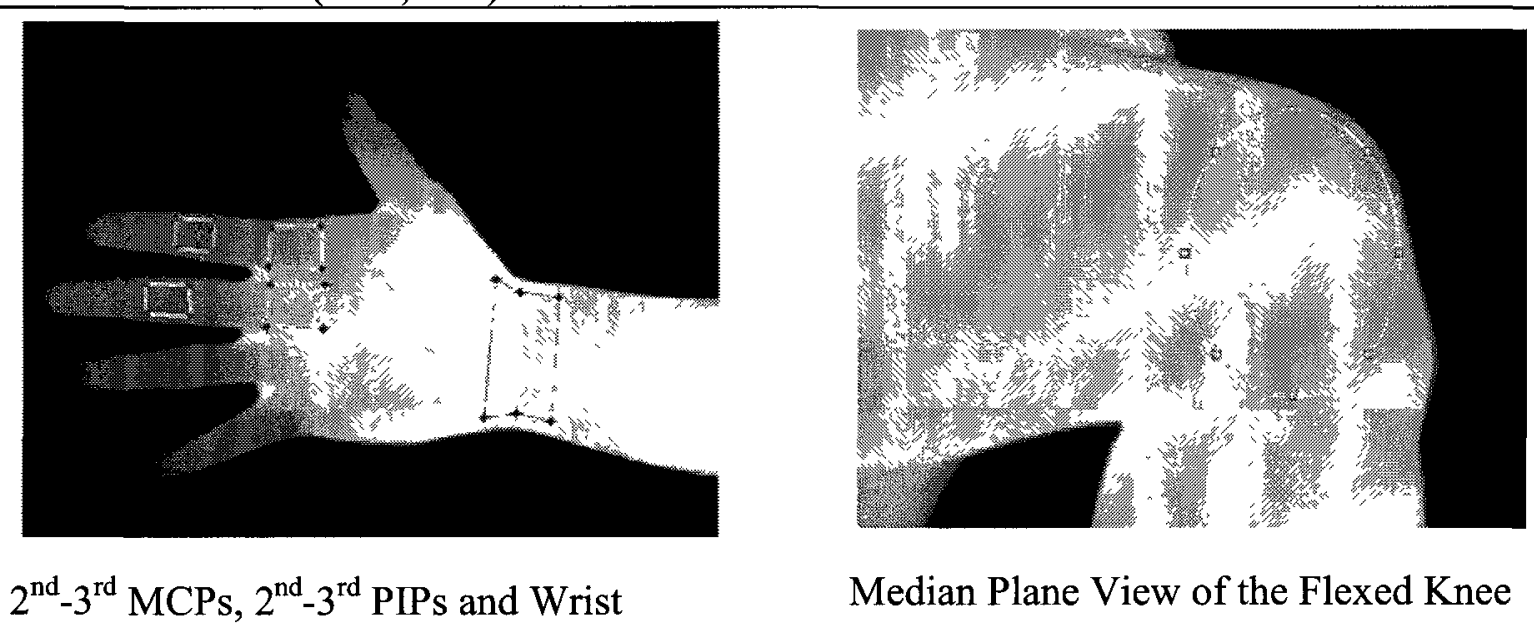

$2^{\text {nd }}-3^{\text {rd }}$ MCPs, $2^{\text {nd }}-3^{\text {rd }}$ PIPs and Wrist

Median Plane View of the Flexed Knee

Once the joints were identified, MATLAB outputted an array of temperature values which represented the temperature of every pixel within the selected region of interest of the TIR image. The resulting array was a probability distribution of a real-valued random variable. 
Measurements extracted from these arrays to be analysed were: max, min, mean, median, mode, standard deviation, skewness and kurtosis. The max and min measurements refer to the maximum and minimum temperatures within the array of the joint. The mean is the average temperature value of the elements of the array. The median is the temperature value of the middle element of the joint's array when all the elements of the array are arranged in either ascending or descending order according to their temperature values. The mode is the temperature value that occurs most frequently in the array of the joint. The mean, median and mode are measures of central tendency, the location of a distribution. The standard deviation is a measure of dispersion and refers to the variability of the array distribution around the mean value. The skewness and the kurtosis describe the shape of the distribution. The skewness is a measure of the asymmetry of an array's distribution. The kurtosis is a description of the peak of the array's distribution (Salvatore 2002).

From the literature review it has been established that certain measurements (such as max, min, mean, median, and mode) extracted from the TIR images are subject to interparticipant variation and many other factors (such as time of day, vascular distribution of the patient, environment, etc.). Therefore, in order to eliminate the possibility that a person might have warmer or colder temperatures than another, these measurements were slightly modified. This measurement modification was inspired by the Heat Distribution Index and Salisbury's research, where ratios, differences and dispersion measurements (variance) were used to compare the measurements of the control subjects and the patients. These modified measurements were based on the thermal distribution of the surface of the skin rather than the exact temperature reading, thus eliminating inter-participant differences (Salisbury 1983). Thus the modified datasets used for the analysis were: 
Skewness

Kurtosis

Variance

Mode/Max

Median/Max

Min/Max
Max-Min

(Mode - Mean)2

Mode/Min

Median/Min

Mean/Min

Salisbury's HDI is based on the dispersion (the variance) of the distribution of the thermal surface over the joint. In this thesis, many other measures of dispersion are used to characterize different dispersion aspects of the datasets. For instance, the skewness and kurtosis are direct representations of the temperature distribution's shape and thus indirectly represents the dispersion. The variance is the standard deviation (STD) squared and is the typical measurement of dispersion. The (Mode-Mean) ${ }^{2}$ is the mode temperature minus the mean temperature squared. It represents the dispersion range between the mode temperature and the mean temperature.

To further explore datasets that eliminate inter-participant variation, ratios were also explored: Mode/Max, Median/Max, Mode/Min, Median/Min and Mean/Min are calculated by dividing the numerator temperature (by the mode, median or mean) by the denominator temperature (by either max or min). These ratios normalize the exact temperature value with the participant's own maximum or minimum temperature value. These ratios eliminated the variations caused by a naturally colder or warmer participant.

The Max-Min datasets is the maximum temperature minus the minimum temperature and represents the temperature range of the joint region. This range is explored to see if the datasets can be used to correctly represent the temperature range of control subjects and RA patients (Adéa, 2009). 
The hypothesis is that the dispersion, ratio and difference datasets may be a better representation of the presence or absence of RA in participants.

\subsubsection{Lilliefors Test}

To complete the statistical analysis, the first step involved testing the distribution of the datasets for normality. If the datasets are not distributed normally, then the use of statistical tests derived from the normal distribution such as the t-test and ANOVA are invalid (Salvatore, 2002). In this current analysis, the Lilliefors Test of normality was used (Matlab, 2009, Adéa 2009). It was applied to the dataset series to determine if they were Gaussian. The result from this analysis helped in determining if the null hypothesis tests were applicable to the dataset. In order to accomplish the first objective stated, these hypothesis tests should show that the patients datasets were statistically different from the control subjects datasets at a significant level of at least $5 \%(\alpha=0.05)$ and with a p-value lower than 0.05 . The values selected for $\alpha$ and $p$ are the generally accepted values for statistical significance in hypothesis testing (Salvatore, 2002). This is used to test the null hypothesis that the datasets samples come from a normal distribution against the alternative that they do not come from a distribution in the normal family. It is a 2sided goodness-of-fit test used when the parameters of the distribution are unknown and must be estimated. The Lilliefors test statistic is:

$$
K S=\max _{x}|S C D F(x)-C D F(x)|
$$

where SCDF is the cumulative distribution function taken from the datasets samples and CDF is the normal cumulative distribution function with the same mean and standard deviation as the sample datasets (Matlab, 2009). 
The lillietest function in MATLAB uses a table of critical values computed using Monte Carlo simulation for sample sizes less than 1000 and significance levels between 0.001 and 0.50 . The significance level $(\alpha)$ is used to determine the region of rejection of the statistical test (critical value). If Lilliefors test statistic $>$ critical value, then the null hypothesis is rejected. If Lilliefors test statistic < critical value, then the null hypothesis cannot be rejected (Salvatore, 2002). MATLAB outputs four results: $h$ value, $p$ value, $K S$ value and critical value. The test returns the logic value $h=1$ if the null hypothesis is rejected and 0 if it cannot be rejected at the $5 \%$ significance level. The significance level was always set to 0.05 in this thesis, to establish the same significance level used in previous work. The $\mathrm{p}$ value is the probability of observing an element from the datasets distribution if the null hypothesis is true. The KS value is the Lilliefors test statistic retrieved from the equation stated above. The critical value is the value that determines the rejection region of the test based on $\alpha$ and the degrees of freedom.

\subsubsection{Kruskal-Wallis Test and Ranksum Test}

After the normality test had been completed, the second step of the statistical analysis was applying hypothesis tests to the datasets. If the datasets were distributed normally, then parametric hypothesis tests such as the Student t-Test and the Anova test were be used to prove the statistical significance of the results. If the datasets were not distributed normally, then parametric hypothesis test were not applicable, then non-parametric hypothesis tests, such as the Kruskal-Wallis Test (Salvatore, 2002; Matlab, 2009) and the Ranksum Test (Matlab, 2009), were used to prove the statistical significance of the results. Every dataset from the control group was compared to every dataset from the patient group for every joint using MATLAB'S ranksum test, and then the results were confirmed with the Kruskal-Wallis test. This test performed a twosided non-parametric test of the null hypothesis that data in the two samples were independent 
samples from identical continuous distributions with equal medians, against the alternative that they do not have equal medians.

\subsection{Classification Methodology}

After the first objective of the thesis was accomplished, the next step was to use these dataset series from the established joints that showed statistical difference in the datasets of the control subjects and patient groups as input variables into a classifier. The goal behind this, was to create a data mining approach that would generate a classifier that improved recognition of both positive cases; presence of RA (sensitivity) and negative cases; no RA (specificity) and also showed the effect of the sample size on the classifier; and generated a classifier that showed which joints were good indicators of RA and compared the combination of 12 dataset series to determine which combination were the best discriminator. And lastly, the determination of the level of activity of RA in each patient, where they were classified into three classes: LOW, MEDIUM and HIGH.

\subsubsection{Accomplishing Objective \#2}

The second objective was to discriminate between patients and control subjects. The goal was, using the various statistical dataset series (Min and Max temp, Min/Max and Median/Max, Mode/Min and Median/Min, Mode/Min and Mean/Min, Mode/Max and Median/Max, Mode/Max and Mean/Max, Min/Max and Median/Min, Min/Max and Mean/Min, Min/Max and Mean/Min, Median/Max and Mode/Min, Mean/Max and Min/Max, Min/Max and Mode/Min and Mode/Min and Median/Max) to establish which of the datasets, if any, were able to generate a classifier that could discriminate with a high enough specificity and sensitivity (over $85 \%$ 
respectively) between the patient and control subject groups, and to also determine the effect of increase in the sample size on the classifier generated. To accomplish this task, the following steps were followed for each dataset series combination.

Step 1 Separate datasets into different dataset combination series. Establish the risk model. Step 2 Input variables into the DT.

Step 3 Determine results of the DT

- Split each database into a train set (TRAIN) and test set (TEST). Bootstrap the TRAIN set to create five bootstrapped datasets b_TRAIN.

- Classify Patients and Control subjects using DTs.

\subsubsection{Accomplishing Objective \#3}

The third objective was to assess which joints were more affected than others, in order to help target certain joints in terms of diagnosis, because each person's degree of severity in certain joint vary from one another. This was done in two ways, first the combination of joints that generated the best classifier was determined by a process of elimination of variables (joints) and alternations; the second step was to separate these joints individually to determine which joints created the best classifier indicating the presence of RA. Then the various dataset series were compared to determine which combination helped improve the classifications sensitivity and specificity.

Step 1 Separate datasets into different dataset combination series. Establish the risk model.

Step 2 Input variables into the DT.

Step 3 Determine results of the DT

- Split each database into a train set (TRAIN) and test set (TEST). Bootstrap the TRAIN set to create five bootstrapped datasets b_TRAIN.

- Classify Patients Joints DTs. 


\subsubsection{Accomplishing Objective \#4}

The fourth objective was to classify the level of severity of RA. After successfully being able to discriminate between the patients and the control subjects, the next step was generating a classifier that could determine the level of severity in the patient groups and separate them into three classes: LOW, MEDIUM and HIGH, using the same dataset series previously used to satisfy the second objective. However, it is important to note that only those dataset series established to be the best discriminators from the second objective were used to generate the classifiers. As in the previous objectives, the following steps were followed:

Step 1 Separate datasets into different dataset combination series. Establish the risk model. Step 2 Input variables into the DT.

Step 3 Determine results of the DT

- Split each database into a train set (TRAIN) and test set (TEST). Bootstrap the TRAIN set to create five bootstrapped datasets b_TRAIN.

- Classify Patients into LOW, MEDIUM, HIGH levels of severity using DTs.

\subsection{Creating DT Input Variables}

\subsubsection{Input Variables for DT}

The initial variable list, as shown in the table below (Table 3-6), pertains to variables that were submitted to the See5 Decision Tree for feature selection. Additional predictor variables discovered during initial data processing steps and suspected to improve classification were also included. The degree of importance of these variables varied according to their attribute usage: Some of the most prominent variables were the mobility, in terms of range of motion that could be comfortably reached, the number of years the disease has been present and race. Race proved 
to be an important aspect of the classification process, as it was observed during temperature measurement extraction that the participants with black ethnic backgrounds had a slightly higher temperature measurement than those from other races (Caucasian or Asian descent). Some of these variables were extracted from the questionnaire (Appendix A).

Table 3-6: Shows the variables submitted to the See5 software and their description

\begin{tabular}{ll} 
VARIABLE & \multicolumn{1}{c}{ DESCRIPTION } \\
\hline \hline PART & Participant \# \\
SEX & Gender (Male or Female) \\
AGE & Age (Years) \\
FRP & Flare up period (True or False) \\
MEDS & Medications on \\
SURG & Surgical Intervention (True or False) \\
ALT_THER & Alternative therapy \\
ACT_LEV & Activity level (lifestyle) \\
MOB & Level of mobility \\
MORN_STIFF & Morning stiffness \\
YEARS & Years with diseases \\
TIME & Time of day of imaging session \\
KNEE & Knee joint \\
MCP2 & 2nd Metacarpophalangeal joint \\
MCP3 & 3rd Metacarpophalangeal joint \\
ANK & Ankle Joint \\
BLACK & Black \\
WHITE & White \\
ASIAN & Asian \\
OTH_ISS & Other (Any) joint issues \\
\hline \hline
\end{tabular}

\subsubsection{Dividing the Dataset}

The whole dataset was split into a training and test set: $70 \%$ of the data was allocated for the training (TRAIN) portion and tree generation and $30 \%$ of the data was used for the testing portion (TEST) of the tree classifier. The training set was bootstrapped three times (using about $90 \%$ ), creating the bootstrapped training subsamples (bTRAIN). 


\subsubsection{Ensemble Decision Tree}

Redundant or similar data that came from the statistical analysis were used for the initial DT classifier. All these variables were used to generate the first DT. See5 displays which attributes have the most contribution to the classifier in terms of percentage. The steps used in the creation of each of the three DT were as follows:

1. Create a DT using all the predictor variables found in Table including confounding variables.

2. Attributes that contribute to less than $1 \%$ to the classifier are not displayed in the attribute usage list. We ignore these variables when creating the next tree - ignoring the variable with the least contribution according to the attribute usage.

3. Re-generate a new tree using the modified variable subset list.

4. Remove each variable iteratively before regenerating a tree until the tree has optimal sensitivity, specificity and minimal error. 


\section{CHAPTER FOUR}

\section{RESULTS AND DISCUSSION}

This chapter begins with an analysis of the thermal images, and then discusses the resulting statistical data. It then shows the classification of the thermal images using decision trees and comparing the best dataset series that helps improve classification. The chapter concludes with a discussion of the results and discussion of whether the thesis objectives stated were rejected or confirmed.

\subsection{Results}

In this chapter, all raw data measurements extracted from the original Thermal InfraRed (TIR) images can be found in Appendix D. The datasets used for analysis and classification purposes are all calculated from the raw measurements. All the classification decision tree results for the datasets can be found in Appendix E.

The table below shows the average value for each dataset of every joint for the control and patient groups. 
Table 4-1: Average values for each dataset of each joint for the patient and control groups

\begin{tabular}{|c|c|c|c|c|c|c|c|c|c|c|}
\hline \multicolumn{11}{|c|}{ Average Values for: } \\
\hline Joints & & Variance & Mode/Max & Median/Max & Min/Max & Max-Min & (Mode-Mean)2 & Mode/Min & Median/Min & Mean/Min \\
\hline & Controls & 0.6397 & 0.9950 & 0.9951 & 0.9891 & 3.3049 & 0.3933 & 1.0059 & 1.0060 & 1.0060 \\
\hline Ankle & Patients & 0.7559 & 0.9944 & 0.9947 & 0.9875 & 3.8072 & 0.5447 & 1.0070 & 1.0072 & 1.0070 \\
\hline & Controls & 0.3594 & 0.9889 & 0.9958 & 0.9896 & 3.1589 & 0.1298 & 1.0067 & 1.0062 & 1.0060 \\
\hline Knee & Patients & 0.4650 & 0.9893 & 0.9964 & 0.9911 & 2.6986 & 0.2694 & 1.0054 & 1.0054 & 1.0051 \\
\hline & Controls & 0.1400 & 0.9964 & 0.9968 & 0.9943 & 1.7248 & 0.0492 & 1.0022 & 1.0025 & 1.0026 \\
\hline 2nd MCP & Patients & 0.1421 & 0.9972 & 0.9971 & 0.9938 & 1.8992 & 0.0435 & 1.0035 & 1.0034 & 1.0033 \\
\hline & Controls & 0.0883 & 0.9911 & 0.9974 & 0.9955 & 1.3563 & 0.0442 & 1.0015 & 1.0019 & 1.0019 \\
\hline 3rd MCP & Patients & 0.0628 & 0.9933 & 0.9978 & 0.9962 & 1.1577 & 0.0288 & 1.0016 & 1.0016 & 1.0017 \\
\hline & Controls & 0.0762 & 0.9977 & 0.9979 & 0.9958 & 1.2555 & 0.0495 & 1.0019 & 1.0021 & 1.0021 \\
\hline 2nd PIP & Patients & 0.0707 & 0.9978 & 0.9980 & 0.9955 & 1.3603 & 0.1521 & 1.0022 & 1.0025 & 1.0025 \\
\hline & Controls & 0.0794 & 0.9982 & 0.9983 & 0.9956 & 1.3230 & 0.1320 & 1.0026 & 1.0027 & 1.0026 \\
\hline 3rd PIP & Patients & 0.0750 & 0.9983 & 0.9982 & 0.9952 & 1.4747 & 0.0209 & 1.0031 & 1.0030 & 1.0030 \\
\hline & Controls & 0.1376 & 0.9974 & 0.9973 & 0.9941 & 1.8068 & 0.0538 & 1.0033 & 1.0033 & 1.0032 \\
\hline Wrist & Patients & 0.1619 & 0.9971 & 0.9969 & 0.9934 & 2.0290 & 0.0507 & 1.0038 & 1.0036 & 1.0035 \\
\hline & Controls & 0.1041 & 0.9979 & 0.9978 & 0.9955 & 1.3599 & 0.0526 & 1.0024 & 1.0023 & 1.0023 \\
\hline 1st MTP & Patients & 0.0963 & 0.9978 & 0.9978 & 0.9958 & 1.2687 & 0.0633 & 1.0022 & 1.0024 & 1.0021 \\
\hline & Controls & 0.0690 & 0.9979 & 0.9980 & 0.9961 & 1.1570 & 0.0494 & 1.0018 & 1.0019 & 1.0019 \\
\hline 5th MTP & Patients & 0.0681 & 0.9981 & 0.9982 & 0.9961 & 1.1607 & 0.0225 & 1.0020 & 1.0021 & 1.0020 \\
\hline & Controls & 0.4018 & 0.9955 & 0.9973 & 0.9906 & 2.8615 & 0.2110 & 1.0049 & 1.0067 & 1.0048 \\
\hline Elbow & Patients & 0.4428 & 0.9954 & 0.9953 & 0.9902 & 3.0029 & 0.2201 & 1.0053 & 1.0052 & 1.0051 \\
\hline
\end{tabular}

*Participants $9 \& 10$ (control subjects) data were excluded from the group

From the above table, it is important to note that for the control group's averages the calculation from 52 datasets values (26 control subjects $\mathrm{x} 2$ ), because each control subject presented two TIR images per joint - the left joint and the right joint. Also for the patient group's averages, the same approach was used; they were calculated from 52 datasets values (26 RA patients $\mathrm{x} 2$ ). This is important to note, because as with the nature of RA, it is an auto-immune disease that affects the joints bilaterally - meaning the disease is present in the joints on both sides of the body. For instance, if the right wrist is affected by RA, the left wrist would also be affected by RA. 
The reason for the exclusion of participants 9 and 10 (control subjects) from the research was because those participants showed exceedingly higher temperatures than those observed in the normals. This led to their exclusion because, from their results, even though they were not diagnosed with RA, these abnormally high temperature ranges could possibly be attributed to potential joint issues that were yet to be diagnosed at the time of the study or it could also be attributed to the fact that these participants did not strictly adhere to the infrared imaging protocol (see section 3.3.3), with various environmental factors influencing their temperature measurements. It is also important to note that their results were excluded from both the statistical analysis and the classification for the purpose of consistency.

\subsection{Statistical Analysis}

To complete the statistical analysis described in chapter 3, Lilliefors test and Ranksum test were performed on the datasets. All the statistical explanations and equations provided in this chapter were derived from MathWork's MATLAB Help files and from D. Salvatore and D. Reagle's "Statistics and Econometrics" book from the Schaum's Outline Series of McGraw-Hill Companies (2002).

\subsubsection{FIRST OBJECTIVE-determining the joints that statistically discriminate best between the patients and control subjects}

To accomplish this first objective, the first step was determining whether the data samples calculated from the TIR measurements came from a normal distribution. This was determined by using the Lillietest function in MATLAB. So, random datasets for random joints were selected to test for normality using MATLAB's lillietest function. The table below shows MATLAB's four outputs obtained by the normality test. From this table we can conclude that the 
datasets distribution is not from a normal family, the table shows that $h=1$ and the $p$ values are lower than $\alpha(\alpha=0.05)$; therefore rejecting the null hypothesis.

Table 4-2: MATLAB Lilliefors Test Results for Randomly Selected Datasets

\begin{tabular}{|c|c|c|c|c|c|c|}
\hline \multicolumn{7}{|c|}{ Matlab Lilliefor test for randomly selected joints } \\
\hline \multirow[t]{2}{*}{$\begin{array}{l}\text { Participant } \\
\text { Type } \\
\end{array}$} & \multirow[t]{2}{*}{ Joint } & \multirow[t]{2}{*}{ Dataset } & \multicolumn{4}{|c|}{ MATLAB Outputs } \\
\hline & & & $\begin{array}{l}\mathrm{h} \\
\text { Value } \\
\end{array}$ & $\begin{array}{l}\mathbf{P} \\
\text { Value } \\
\end{array}$ & $\begin{array}{l}\text { KS } \\
\text { Value } \\
\end{array}$ & $\begin{array}{l}\text { Critical } \\
\text { Value } \\
\end{array}$ \\
\hline \multirow{9}{*}{$\begin{array}{l}\text { Control } \\
\text { Subjects }\end{array}$} & Ankle & Skewness & 1 & 0.0010 & 0.1456 & 0.1620 \\
\hline & 1st MTP & Kurtosis & 1 & 0.0216 & 0.3777 & 0.1620 \\
\hline & 3rd MTP & Kurtosis & 1 & 0.0031 & 0.2146 & 0.1620 \\
\hline & Elbow & Max Temperature & 1 & 0.0150 & 0.1952 & 0.1620 \\
\hline & 1st PIP & Min Temperature & 1 & 0.0002 & 0.1927 & 0.1620 \\
\hline & 3rd PIP & Variance & 1 & 0.0024 & 0.1771 & 0.1620 \\
\hline & 2nd MCP & Variance & 1 & 0.0121 & 0.2399 & 0.1620 \\
\hline & 3rd MCP & Max Temperature & 1 & 0.0056 & 0.1990 & 0.1620 \\
\hline & Knee & Skewness & 1 & 0.0198 & 0.1782 & 0.1620 \\
\hline \multirow{9}{*}{ Patients } & Ankle & Kurtosis & 1 & 0.0515 & 0.3110 & 0.2132 \\
\hline & 1st MTP & Variance & 1 & 0.0037 & 0.2654 & 0.2132 \\
\hline & 3rd MTP & Kurtosis & 1 & 0.0451 & 0.4142 & 0.2132 \\
\hline & Elbow & Min Temperature & 1 & 0.0040 & 0.1664 & 0.2132 \\
\hline & 1st PIP & Max Temperature & 1 & 0.0379 & 0.1999 & 0.2132 \\
\hline & 3rd PIP & Skewness & 1 & 0.0606 & 0.1846 & 0.2132 \\
\hline & 2nd MCP & Max Temperature & 1 & 0.0210 & 0.4005 & 0.2132 \\
\hline & 3rd MCP & Skewness & 1 & 0.0111 & 0.3792 & 0.2132 \\
\hline & Knee & Kurtosis & 1 & 0.0001 & 0.2444 & 0.2132 \\
\hline
\end{tabular}

The results from above are of the Lilliefors test performed on eighteen datasets representing the thermal distribution of the joints, and they were shown not to be normally distributed (since $h=1$ in all datasets) at a 5\% significance level $(\alpha=0.05)$. The $K S$ values were all superior to the critical values and the p-values are all lower than $\alpha$; from this it can be confirmed and concluded that the datasets are not from the normal Gaussian family. Therefore, for the purposes of this thesis, it would be assumed from here on that all the datasets are not 
normally distributed, since the eighteen randomly selected datasets do not show normality. As a result, as stated earlier, parametric hypothesis tests cannot be used for the statistical analysis. Instead of the Student t-Test and the Anova Test, the Kruskal-Wallis Test and the Ranksum Test are applied to the datasets.

The next step was to show that the control group's datasets were statistically different from the patient's datasets, in essence, showing that TIR images could be used to discriminate between the control and patient groups. Since it had been established that the datasets were not normally distributed, non-parametric hypothesis test were used to show this. The Kruskal-Wallis test and the Ranksum test were implemented for this purpose. The Kruskal-Wallis test is a nonparametric version of the classical one-way ANOVA, and an extension of the Wilcoxon ranksum test for more than two groups. This test is based on the standard Anova table using ranks rather than the numeric values of individual sample points. The function compares the medians of the samples, and returns the $p$-value for the null hypothesis that all samples are drawn from the same population (or equivalently, from different populations with the same distribution) (Matlab 2009).

MATLAB's ranksum test, also a non-parametric test, is equivalent to a Mann-Whitney $U$-test, and is also similar to the Kruskal-Wallis test. It is a two sided non-parametric test with the null hypothesis that two datasets are independent samples from identical continuous distributions with equal medians, against the alternative that they do not have equal medians. If the null hypothesis of the Kruskal-Wallis and the Ranksum tests are rejected at a significance level of $5 \%$ with a p-value lower than 0.05 , it is fair to say that the two compared datasets are not from the same distribution and are statistically different (Matlab, 2009). 
MATLAB's ranksum test outputs two results: $h$ value, $p$ value. The $h$ value is 1 if the null hypothesis is rejected and 0 if it cannot be rejected at the $5 \%$ significance level. The $p$ value is the probability of observing an element from the datasets distribution if the null hypothesis is true. If the $h$ value is 1 , with a p-value lower than 0.05 , it is possible to conclude that the datasets of the control group are statistically different from the datasets of the patient group (Matlab, 2009). The table below demonstrates the two MATLAB outputs from the ranksum test performed on the two groups (control subjects and patients) datasets distribution.

Table 4-3: MATLAB's Ranksum Test Results Comparing the Datasets of the Control and the Patient Group

\begin{tabular}{|c|c|c|c|c|c|c|c|}
\hline \multicolumn{8}{|c|}{ MATLAB Ranksum Test Results comparing the Datasets of the control and patients } \\
\hline Joint & Datasets & $\begin{array}{l}\mathbf{P} \\
\text { Value }\end{array}$ & $\begin{array}{l}\mathrm{h} \\
\text { value }\end{array}$ & Joint & Datasets & $\begin{array}{l}\mathbf{P} \\
\text { Value }\end{array}$ & $\begin{array}{l}\mathbf{h} \\
\text { value }\end{array}$ \\
\hline \multirow{12}{*}{ Ankle } & STD & 0.7562 & 0 & \multirow{12}{*}{ 1st MTP } & STD & 0.0235 & 1 \\
\hline & Skewness & 0.3411 & 0 & & Skewness & 0.0113 & 1 \\
\hline & Kurtosis & 0.2453 & 0 & & Kurtosis & 0.7654 & 0 \\
\hline & Mode/Max & 0.9842 & 0 & & Mode/Max & 0.6752 & 0 \\
\hline & Median/Max & 0.0011 & 1 & & Median/Max & 0.7550 & 0 \\
\hline & Min/Max & 0.0034 & 1 & & Min/Max & 0.3511 & 0 \\
\hline & Variance & 0.0167 & 1 & & Variance & 0.0253 & 1 \\
\hline & Max-Min & 0.0451 & 1 & & Max-Min & 0.0026 & 1 \\
\hline & (Mode - Mean) 2 & 0.2451 & 0 & & (Mode - Mean)2 & 0.9880 & 0 \\
\hline & Mode/Min & 0.1675 & 0 & & Mode/Min & 0.2227 & 0 \\
\hline & Median/Min & 0.0063 & 1 & & Median/Min & 0.4710 & 0 \\
\hline & Mean/Min & 0.0411 & 1 & & Mean/Min & 0.5634 & 0 \\
\hline Joint & Datasets & $\begin{array}{l}\mathbf{P} \\
\text { Value }\end{array}$ & $\begin{array}{l}\mathbf{h} \\
\text { value }\end{array}$ & Joint & Datasets & $\begin{array}{l}\mathbf{P} \\
\text { Value }\end{array}$ & $\begin{array}{l}\mathbf{h} \\
\text { value }\end{array}$ \\
\hline \multirow{9}{*}{ 1st PIP } & STD & 0.2349 & 0 & \multirow{9}{*}{ 2nd PIP } & STD & 0.7217 & 0 \\
\hline & Skewness & 0.1154 & 0 & & Skewness & 0.5622 & 0 \\
\hline & Kurtosis & 0.6799 & 0 & & Kurtosis & 0.5001 & 0 \\
\hline & Mode/Max & 0.1000 & 0 & & Mode/Max & 0.3461 & 0 \\
\hline & Median/Max & 0.5644 & 0 & & Median/Max & 0.1286 & 0 \\
\hline & Min/Max & 0.9888 & 0 & & Min/Max & 0.5627 & 0 \\
\hline & Variance & 0.3423 & 0 & & Variance & 0.0631 & 0 \\
\hline & Max-Min & 0.7787 & 0 & & Max-Min & 0.1117 & 0 \\
\hline & (Mode - Mean)2 & 0.3212 & 0 & & (Mode - Mean)2 & 0.9451 & 0 \\
\hline
\end{tabular}




\begin{tabular}{|c|c|c|c|c|c|c|c|}
\hline & Mode/Min & 0.4467 & 0 & & Mode/Min & 0.5400 & 0 \\
\hline & Median/Min & 0.0654 & 0 & & Median/Min & 0.1005 & 0 \\
\hline & Mean/Min & 0.2354 & 0 & & Mean/Min & 0.2911 & 0 \\
\hline Joint & Datasets & $\begin{array}{l}\mathbf{P} \\
\text { Value } \\
\end{array}$ & $\begin{array}{l}\mathrm{h} \\
\text { value }\end{array}$ & \multirow{13}{*}{$\begin{array}{l}\text { 2nd } \\
\text { MCP }\end{array}$} & Datasets & $\begin{array}{l}\mathbf{P} \\
\text { Value } \\
\end{array}$ & $\begin{array}{l}\mathrm{h} \\
\text { value } \\
\end{array}$ \\
\hline \multirow{12}{*}{ 3rd PIP } & STD & 0.7511 & 0 & & STD & 0.5644 & 0 \\
\hline & Skewness & 0.3981 & 0 & & Skewness & 0.0033 & 1 \\
\hline & Kurtosis & 0.0047 & 1 & & Kurtosis & 0.8722 & 0 \\
\hline & Mode/Max & 0.7770 & 0 & & Mode/Max & 0.0176 & 1 \\
\hline & Median/Max & 0.3344 & 0 & & Median/Max & 0.3429 & 0 \\
\hline & Min/Max & 0.3961 & 0 & & Min/Max & 0.2277 & 0 \\
\hline & Variance & 0.4859 & 0 & & Variance & 0.1058 & 0 \\
\hline & Max-Min & 0.7216 & 0 & & Max-Min & 0.2592 & 0 \\
\hline & (Mode - Mean) 2 & 0.0782 & 0 & & (Mode - Mean) 2 & 0.3366 & 0 \\
\hline & Mode/Min & 0.4455 & 0 & & Mode/Min & 0.0097 & 1 \\
\hline & Median/Min & 0.2786 & 0 & & Median/Min & 0.2145 & 0 \\
\hline & Mean/Min & 0.0882 & 0 & & Mean/Min & 0.2365 & 0 \\
\hline Joint & Datasets & $\begin{array}{l}\text { P } \\
\text { Value }\end{array}$ & $\begin{array}{l}\text { h } \\
\text { value }\end{array}$ & Joint & Datasets & $\begin{array}{l}\mathbf{P} \\
\text { Value }\end{array}$ & $\begin{array}{l}\mathrm{h} \\
\text { value }\end{array}$ \\
\hline \multirow{12}{*}{$\begin{array}{l}\text { 3rd } \\
\text { MCP }\end{array}$} & STD & 0.0175 & 1 & \multirow{12}{*}{ Knee } & STD & 0.0235 & 1 \\
\hline & Skewness & 0.3586 & 0 & & Skewness & 0.4555 & 0 \\
\hline & Kurtosis & 0.7091 & 0 & & Kurtosis & 0.4120 & 0 \\
\hline & Mode/Max & 0.0312 & 1 & & Mode/Max & 0.7231 & 0 \\
\hline & Median/Max & 0.0101 & 1 & & Median/Max & 0.6666 & 0 \\
\hline & Min/Max & 0.0345 & 1 & & Min/Max & 0.0035 & 1 \\
\hline & Variance & 0.0175 & 1 & & Variance & 0.0253 & 1 \\
\hline & Max-Min & 0.0008 & 1 & & Max-Min & 0.0145 & 1 \\
\hline & (Mode - Mean)2 & 0.0245 & 1 & & (Mode - Mean)2 & 0.8971 & 0 \\
\hline & Mode/Min & 0.2019 & 0 & & Mode/Min & 0.0222 & 1 \\
\hline & Median/Min & 0.0400 & 1 & & Median/Min & 0.0147 & 1 \\
\hline & Mean/Min & 0.0115 & 1 & & Mean/Min & 0.0045 & 1 \\
\hline Joint & Datasets & $\begin{array}{l}\text { P } \\
\text { Value } \\
\end{array}$ & $\begin{array}{l}\text { h } \\
\text { value }\end{array}$ & Joint & Datasets & $\begin{array}{l}\mathbf{P} \\
\text { Value } \\
\end{array}$ & $\begin{array}{l}\mathrm{h} \\
\text { value }\end{array}$ \\
\hline \multirow{7}{*}{ Elbow } & STD & 0.2260 & 0 & \multirow{7}{*}{ Wrist } & STD & 0.3684 & 0 \\
\hline & Skewness & 0.3922 & 0 & & Skewness & 0.2112 & 0 \\
\hline & Kurtosis & 0.7620 & 0 & & Kurtosis & 0.2341 & 0 \\
\hline & Mode/Max & 0.4767 & 0 & & Mode/Max & 0.0642 & 0 \\
\hline & Median/Max & 0.1925 & 0 & & Median/Max & 0.4992 & 0 \\
\hline & Min/Max & 0.3104 & 0 & & Min/Max & 0.2431 & 0 \\
\hline & Variance & 0.7747 & 0 & & Variance & 0.5666 & 0 \\
\hline
\end{tabular}




\begin{tabular}{|l|l|l|l|l|l|}
\hline Max-Min & 0.7324 & 0 \\
\hline (Mode-Mean)2 & 0.4428 & 0 \\
\hline Mode/Min & 0.5960 & 0 \\
\hline Median/Min & 0.0765 & 0 \\
\hline Mean/Min & 0.9267 & 0 & Max-Min & 0.6789 & 0 \\
\cline { 4 - 6 } & (Mode-Mean)2 & 0.7422 & 0 \\
\cline { 2 - 5 } & Mode/Min & 0.4105 & 0 \\
\cline { 2 - 5 } & & Median/Min & 0.3982 & 0 \\
\cline { 2 - 5 } & Mean/Min & 0.5629 & 0 \\
\hline
\end{tabular}

The Kruskal-Wallis test was also performed on the distribution datasets of the two groups and results yield the same results as the ranksum test, thereby verifying the hypothesis test results above.

The table above (Table 4-3) highlighted in blue were the datasets where the null hypothesis was rejected at a significance level of $5 \%$ with a p-value that was lower than $\alpha$. These highlighted test results were the ones that successfully discriminated between the control group and the RA group datasets. Therefore, the first objective, which was to determine the joints that could statistically discriminate between the patients and control subjects, is satisfied since:

1- The Median/Max, Min/Max, variance, Max-Min, Median/Min, Mean/Min of the ankle were statistically different for the control and patient group;

2- The standard deviation (STD), skewness, variance, Max-Min of the $1^{\text {st }}$ MTP were statistically different for the control and patient groups; however, the $1^{\text {st }}$ MTP is not an indicator of RA; rather, it is an indicator of osteoarthritis. It was concluded that even though the $1^{\text {st }}$ MTP was able to discriminate between the two groups, it could also mean that those patients had osteoarthritis;

3- The skewness, Mode/Max, Mode/Min datasets of the $2^{\text {nd }}$ MCP were statistically different distributions for the control and patient group; 
4- The standard deviation (STD), Mode/max, Median/max, Min/max, variance, Max-Min, (Mode-Mean) ${ }^{2}$, Mean/Min datasets of the $3^{\text {rd }}$ MCP showed a statistical difference between the control and patient distributions;

5- The control group's kurtosis datasets of the $3^{\text {rd }}$ PIP was statistically different than the patient's kurtosis datasets of the $3^{\text {rd }}$ PIP;

6- $\quad$ The standard deviation, Min/max, Variance, Max-Min, Mode/Min, Median/Min, Mean/Min datasets of the knee are statistically different distribution for the control and patient group.

\subsection{Classification}

The classifications, construction of the decision tree of the datasets were performed using the Rulequest See5 software. The classifier was generated to determine the presence of RA; to also determine the joints that effectively classified the presence of RA among the joints $\left(2^{\text {nd }}\right.$ and $3^{\text {rd }}$ MCP's, Knee and Ankle) statistically established to discriminate between the patient and control subject groups, by a process of elimination of variable (joints) and to classify the level of severity of RA (into three class groups: LOW, MEDIUM and HIGH).

\subsubsection{SECOND OBJECTIVE-determining the presence of RA using the patients and control subjects dataset series}

The first decision tree (DT) was generated from the dataset of the original participants in the study (Using 13 patients and 16 control subject). This was done in order to determine whether the addition of new participants into the study, had any effect on the classifier; whether the classifier showed an improvement or deteriorated. The result (Table 4-4) of the DT using this 
sample size showed a level of consistent accuracy in terms of its specificity and sensitivity among the five bootstrapped DT's. Although the classifier was able to determine the presence of RA in two groups, it was not the most efficient classifier with its sensitivity ranging from 70 to $72 \%$ and its specificity ranging from 74 to $80 \%$. The variables that varied in the DT's were the AGE and the joints (KNEE, ANKLE, MCP2, and MCP3). The root variable which was the MIN and MAX temperatures remained the same among the five bootstrapped DT's; this was used to create all five bootstrapped DT's. The AGE, KNEE and ANKLE were used to create DT 3 and 4, while the MCP2 and MCP3 were used to create DT 2 and 5.

Table 4-4: Min and Max dataset Decision Tree result (Using 13 patients and 16 control subjects)*

\begin{tabular}{|c|l|r|r|r|r|r|}
\hline \multicolumn{2}{|c|}{ DT \# } & $\mathbf{1}$ & $\mathbf{2}$ & $\mathbf{3}$ & $\mathbf{4}$ & $\mathbf{5}$ \\
\hline \multirow{3}{*}{ Train } & Sensitivity & $\mathbf{7 2 . 2}$ & $\mathbf{7 2 . 0}$ & 71.0 & 70.2 & $\mathbf{7 2 . 2}$ \\
& Specificity & 80.0 & 77.5 & 79.0 & 78.1 & 79.0 \\
& CCR & 74.7 & 76.3 & 75.2 & 74.3 & 75.8 \\
\hline \multirow{3}{*}{ Test } & Sensitivity & 72.1 & 72.2 & 70.0 & 72.2 & 72.2 \\
& Specificity & 79.2 & 79.2 & 74.0 & 77.0 & 80.0 \\
& CCR & 76.5 & 75.2 & 73.4 & 74.2 & 79.5 \\
\hline
\end{tabular}

This same step was repeated for the remaining 11 dataset combination. These datasets were the root variables i.e., Min/Max and Median/Max (Column 1 in Table 4-5), while the AGE and joints (KNEE, ANKLE, MCP2, and MCP3). The table below (Table 4-5) illustrates the results of the DT for the different combinations and compares them with one another. From the table below, it is shown that the Min and Max dataset had the best discriminatory value of the datasets (using 13 patients and 16 control subjects) with its specificity and sensitivity at 80 and $72 \%$ respectively, while the Mode/Min and the Mean/Min had the worst with a specificity and sensitivity of 55 and $45 \%$ respectively. However the Min/Max and Median/Min, Median/Max 
and Mode/Min, and Mode/Max and Mean/Max also proved to be good discriminators, which could determine the presence of RA.

Table 4-5: Table showing the results of the DT combination of 12 different dataset series (Using 13 patients and 16 control subjects)

\begin{tabular}{|l|l|l|}
\hline Dataset & Sensitivity (\%) & Specificity (\%) \\
\hline Min and Max & 72 & 80 \\
\hline Min/Max and Median/Max & 65 & 52 \\
\hline Mode/Min and Median/Min & 53 & 70 \\
\hline Mode/Min and Mean/Min & 45 & 55 \\
\hline Mode/Max and Median/Max & 48 & 59 \\
\hline Min/Max and Median/Min & 69 & 79 \\
\hline Min/Max and Mean/Min & 66 & 63 \\
\hline Median/Max and Mode/Min & 70 & 77 \\
\hline Mean/Max and Min/Max & 50 & 71 \\
\hline Min/Max and Mode/Min & 61 & 45 \\
\hline Mode/Min and Median/Max & 69 & 54 \\
\hline Mode/Max and Mean/Max & 72 & 79 \\
\hline
\end{tabular}

After using the dataset of the original participants (13 patients and 16 participants), the dataset from the newly recruited participants (13 patients and 10 participants) were inputted into the decision tree. The result (Table 4-6) of the DT reflects the increased sample size and the effect this increase had on the classifier. Using the same methodology as that used in the previous DT's (see Table 4-4), the DT was bootstrapped five times and the variables that varied in the DT's were AGE and joints (KNEE, ANKLE, MCP2, and MCP3); DT 3 and 4 were created using AGE, KNEE and ANKLE while DT 3 and 4 were created using MCP2 and MCP3. As 
was in the previous DT, the Min and Max dataset had the highest specificity and sensitivity at 92 and $96 \%$ respectively. Comparing the results of the Min and Max dataset, the conclusion can be reached that the larger sample size generated a classifier that could excellently discriminate between the patients with RA and the control subjects without RA.

Table 4-6: Min and Max dataset Decision Tree result (Using 26 patients and 26 control subjects)*

\begin{tabular}{|c|l|r|r|r|r|r|}
\hline \multicolumn{1}{|c|}{ DT \# } & $\mathbf{1}$ & $\mathbf{2}$ & $\mathbf{3}$ & $\mathbf{4}$ & $\mathbf{5}$ \\
\hline \multirow{3}{*}{ Train } & Sensitivity & 100 & 95.3 & 91.0 & 94.4 & 90.0 \\
& Specificity & 93.2 & 92.7 & 88.7 & 86.0 & 87.5 \\
& CCR & 94.6 & 92.0 & 88.6 & 90.2 & 87.7 \\
\hline \multirow{3}{*}{ Test } & Sensitivity & 90.2 & 90.6 & 91.5 & 92.0 & 96.1 \\
& Specificity & 88.9 & 86.5 & 92.3 & 94.4 & 92.5 \\
& CCR & 89.4 & 87.6 & 90.7 & 92.2 & 94.5 \\
\hline
\end{tabular}

As was done with the original sample size, the same steps were repeated for the modified sample size; five bootstrapped DT's were generated for the remaining combination of 11 other dataset series. The results for the DT's are shown in the table below (Table 4-7). This table illustrates the efficiency/accuracy of the classifiers and then compares them against one another. As was expected, the Min and Max dataset showed the highest accuracy amongst the classifiers with a sensitivity and specificity of 96 and $92 \%$ respectively. Also the Min/Max and Median/Max; Mode/Min and Median/Min; Min/Max and Median/Min; and Mode/Max and Mean/Max also proved to be excellent discriminators with a sensitivity that ranged from 89 $96 \%$ and a specificity that ranged from $87-92 \%$. Another interesting observation was from the Min/Max and Median/Max and Mode/Min and Median/Min series; these two dataset combinations proved to be excellent discriminators with the increased sample size ( 26 patients and 26 participants) but did not show much of a discriminatory value with the original sample size (13 patients and 16 control subjects). Even though some of the other dataset series proved to 
be fairly efficient discriminators with a specificity and sensitivity at or above $80 \%$, they were excluded from the highlighted dataset series that proved to be the best indicators because compared to the other datasets with a sensitivity at or above $89 \%$ and a specificity at or above $87 \%$; they were weaker. For the purpose of this thesis, a range from $85 \%$ and above was chosen using AUC index (section 2.6.10) which shows the effectiveness of a classifier according to the level of the AUC index. It appears that results of $85 \%$ or higher are excellent. As was seen with the Min/Max and Median/Max and Mode/Min and Median/Min series, it can be concluded that with a larger sample size there could be an improvement to the classifier generated for the Mode/Min and Median/Min, and Median/Max and Mode/Min dataset. Comparing the results from the classifier using the original dataset (13 patients and 16 control subjects) and the modified dataset ( 26 patients and 26 control subjects), the observation was made that the increase in sample size helped improve the discriminatory value of the classifier, for instance, in the Min and Max dataset, there was an increase in sensitivity from 72 to $96 \%$ and the specificity increased from 80 to $92 \%$. The same observation was made in all the other dataset series also. Increasing the number of points in the datasets seems to have helped in a better training of the classifier and in reducing the error rate in the classification model. It is not known at this point if additional participants would lead again to better results or whether the number used in this research was sufficient and optimal.

Table 4-7: Table showing the results of the DT combination of 12 different dataset series (Using 26 patients and 26 control subjects)

\begin{tabular}{|l|l|l|}
\hline Dataset & Sensitivity (\%) & Specificity (\%) \\
\hline Min and Max & 96 & 92 \\
\hline Min/Max and Median/Max & 90 & 87 \\
\hline Mode/Min and Median/Min & 94 & 92 \\
\hline Mode/Min and Mean/Min & 80 & 76 \\
\hline
\end{tabular}




\begin{tabular}{|l|l|l|}
\hline Mode/Max and Median/Max & 75 & 70 \\
\hline Min/Max and Median/Min & 95 & 92 \\
\hline Min/Max and Mean/Min & 65 & 60 \\
\hline Median/Max and Mode/Min & 80 & 81 \\
\hline Mean/Max and Min/Max & 79 & 77 \\
\hline Min/Max and Mode/Min & 50 & 50 \\
\hline Mode/Min and Median/Max & 60 & 62 \\
\hline Mode/Max and Mean/Max & 89 & 87 \\
\hline
\end{tabular}

From these results, the following conclusions were made; satisfying the second objective:

1- The Median/Max, Min/Max, Median/Min, Mode/Min, Mode/Max of the various joints (ankle and $2^{\text {nd }}$ and $3^{\text {rd }}$ MCP's that discriminated statistically between the patient and control subjects, also proved to be important in classifier when using the combination of Mode/Min and Median/Min, Min/Max and Median/Max, Min/Max and Median/Min, and Mode/Min and Median/Min.

2- The best dataset combination that effectively determined the presence or absence of RA from the sample were the Min and Max, Mode/Max and Mean/Max, Min/Max and Median/Min, Mode/Min and Median/Min, and Min/Max and Median/Max.

3- Increasing the sample size of the datasets helped improve the classifier generated, showing an increase of $24 \%$ in sensitivity and an increase of $12 \%$ in the sensitivity of the classifier. This improvement could be attributed on more 
available datasets to be used to train the classifier, which in also improved the test set of the classifier.

\subsubsection{THIRD OBJECTIVE-determining the joints that best indicates the presence of $R A$ in patients}

After successfully discriminating between the patient and control subjects, the next step was to determine from the patient datasets which joints best indicated the presence of RA by a process of elimination of variables (joints); this was done in order to determine which combination of joints generated the best classifier and then subsequently determine the specific joints that indicated the presence of RA best. The result (Table 4-8) of the DT showed a level of consistent accuracy in terms of its specificity and sensitivity among the five bootstrapped DT's. The variables that varied in the DT's were the AGE and the joints (KNEE, MCP2, and MCP3). The root variable which was the MIN and MAX temperatures remained the same among the five bootstrapped DT's; this was used to create all five bootstrapped DT's. The AGE, KNEE and MCP2 were used to create DT 1 and 4, while the AGE, KNEE and MCP3 were used to create DT 2, MCP2 and MCP3 were used to create DT 3, and the AGE, KNEE, MCP2 and MCP3 were used to create DT 5 along with the root variable. The Min and Max dataset proved to be the most efficient at determining which joints were the best indicators of RA with a sensitivity of $95 \%$ and specificity of $91 \%$. These same steps were repeated for all the dataset series. 
Table 4-8: Min and Max dataset Decision Tree result for Knee, $2^{\text {nd }}$ MCP and $3^{\text {rd }}$ MCP (Using 26 patients and 26 control subjects)*

\begin{tabular}{|c|l|r|r|r|r|r|}
\hline \multicolumn{2}{|c|}{ DT\# } & $\mathbf{1}$ & $\mathbf{2}$ & $\mathbf{3}$ & $\mathbf{4}$ & $\mathbf{5}$ \\
\hline \multirow{3}{*}{ Train } & Sensitivity & 100 & 100 & 96.3 & 94.0 & 99.6 \\
& Specificity & 92.5 & 88.6 & 90.2 & 88.6 & 94.0 \\
& CCR & 94.3 & 91.4 & 87.5 & 87.2 & 95.8 \\
\hline \multirow{3}{*}{ Test } & Sensitivity & 95.5 & 94.8 & 89.6 & 95.5 & 95.5 \\
& Specificity & 89.9 & 90.5 & 86.5 & 90.3 & 91.2 \\
& CCR & 92.1 & 91.7 & 87.3 & 92.6 & 94.4 \\
\hline
\end{tabular}

Table 4-9: Table showing the results of the DT combination of 12 different dataset series for the Knees, $2^{\text {nd }}$ MCP and $3^{\text {rd }}$ MCP (Using 26 patients and 26 control subjects)

\begin{tabular}{|l|l|l|}
\hline Dataset & Sensitivity (\%) & Specificity (\%) \\
\hline Min and Max & 95 & 91 \\
\hline Min/Max and Median/Max & 79 & 74 \\
\hline Mode/Min and Median/Min & 89 & 85 \\
\hline Mode/Min and Mean/Min & 78 & 69 \\
\hline Mode/Max and Median/Max & 72 & 70 \\
\hline Min/Max and Median/Min & 65 & 69 \\
\hline Min/Max and Mean/Min & 71 & 72 \\
\hline Median/Max and Mode/Min & 66 & 57 \\
\hline Mean/Max and Min/Max & 82 & 78 \\
\hline Min/Max and Mode/Min & 80 & 69 \\
\hline Mode/Min and Median/Max & 90 & 88 \\
\hline Mode/Max and Mean/Max & 92 & 85 \\
\hline
\end{tabular}

The Knees, $2^{\text {nd }} \mathrm{MCP}$ and $3^{\text {rd }} \mathrm{MCP}$ proved to be the best joints that indicated the presence of RA. The combination of the ankle, $2^{\text {nd }} \mathrm{MCP}$ and $3^{\text {rd }} \mathrm{MCP}$ also proved to be a good indicator of RA. The DT for the ankle, $2^{\text {nd }} \mathrm{MCP}$ and $3^{\text {rd }} \mathrm{MCP}$ was created using the same methodology as 
the one used in the first DT. The variables that varied in the DT's were the AGE and the joints (ANKLE, MCP2, and MCP3). The root variable which was the MIN and MAX temperatures remained the same among the five bootstrapped DT's; this was used to create all five bootstrapped DT's. The AGE, ANKLE, MCP2 and MCP3 were used to create DT 1, while the AGE, MCP2 and MCP3 were used to create DT 2, ANKLE and MCP3 were used to create DT 3, and the AGE, ANKLE and MCP3 were used to create DT 4 and 5 along with the root variable.

Table 4-10: Min and Max dataset Decision Tree result for four different joint combinations (Using 26 patients and 26 control subjects)*

\begin{tabular}{|l|l|l|}
\hline Joints & Specificity (\%) & Sensitivity (\%) \\
\hline Knees, MCP 2 \& 3 & 95 & 91 \\
\hline MCP 2,Knees \& Ankle & 65 & 58 \\
\hline MCP 3,Knees \& Ankle & 77 & 74 \\
\hline Ankle, MCP 2 \& 3 & 80 & 76 \\
\hline
\end{tabular}

To further establish/confirm which joints were good indicators of RA, the joints were separated and investigated individually. From this further separation it was established that the $2^{\text {nd }}$ and $3^{\text {rd }}$ MCP's were the joints generated the best classifier, using the Min and Max dataset. The $3^{\text {rd }}$ MCP had the highest sensitivity and specificity at 90 and $88 \%$ respectively; while the joint that showed the worst discriminatory value was the ankle with a sensitivity and specificity of 56 and 61\% respectively (as shown in Table 4-12). The result (Table 4-11) of the DT for the $3^{\text {rd }}$ MCP showed consistent accuracy with a specificity range from 80 to $88 \%$ and a sensitivity range from 88 to $90 \%$ among the five bootstrapped DT's. 
Table 4-11: Min and Max dataset Decision Tree result for the $3^{\text {rd }}$ MCP (Using 26 patients and 26 control subjects)*

\begin{tabular}{|c|l|r|r|r|r|r|}
\hline \multicolumn{1}{|c|}{ DT\# } & 1 & 2 & 3 & 4 & 5 \\
\hline \multirow{3}{*}{ Train } & Sensitivity & 100 & 89.5 & 89.5 & 86.7 & 92.4 \\
& Specificity & 82.4 & 83.1 & 77.2 & 80.0 & 96.5 \\
& CCR & 90.2 & 84.3 & 79.9 & 81.4 & 93.5 \\
\hline \multirow{3}{*}{ Test } & Sensitivity & 88.0 & 88.0 & 90.0 & 90.0 & 90.0 \\
& Specificity & 85.3 & 86.7 & 88.0 & 82.5 & 80.0 \\
& CCR & 84.7 & 85.2 & 88.9 & 84.8 & 83.0 \\
\hline
\end{tabular}

Table 4-12: Using the min and max dataset, table showing the specificity and sensitivity of the DT (Using 26 patients and 26 control subjects)*

\begin{tabular}{|l|l|l|}
\hline Joints & Specificity (\%) & Sensitivity (\%) \\
\hline Knees & 72 & 68 \\
\hline Ankles & 61 & 56 \\
\hline MCP 3 & 90 & 88 \\
\hline MCP 2 & 86 & 80 \\
\hline
\end{tabular}

From these results, the following conclusions were made; hence satisfying the stated objective:

1- The combination of the Knee, $2^{\text {nd }} \mathrm{MCP}$ and $3^{\text {rd }} \mathrm{MCP}$ or the Ankle, $2^{\text {nd }} \mathrm{MCP}$ and $3^{\text {rd }} \mathrm{MCP}$ were the best joints that indicated RA and could successfully discriminate between the patient and control groups.

2- The Min and Max, Mode/Min and Median/Max, Mode/Min and Median/Min, Mode/Max and Mean/Max of the combination of the Knee, $2^{\text {nd }}$ MCP and $3^{\text {rd }}$ MCP were the best datasets that generated excellent classifiers.

3- $\quad$ The best joints that best indicated RA in patients were the $2^{\text {nd }}$ and $3^{\text {td }}$ MCP's; these results confirmed those from previous work (Adea 2009); where it was 
statistically shown that these joints were able to discriminate between the patient and control group dataset. This observation is important because the $2^{\text {nd }}$ and $3^{\text {rd }}$ MCP's are joints in the hands where RA presents itself in the early stages are in these joints.

\subsubsection{FOURTH OBJECTIVE-determining the level of severity in the patient group; classifying them into LOW, MEDIUM and HIGH}

The DT was generated to determine the level of activity in the patient group and separate them into three classes: LOW for those patients who were either in the beginning stages of the disease or for those patients whose disease was in remission, MEDIUM for those patients who were at an intermediate level, who could not be classified as LOW, but could not be classified as HIGH either, and HIGH for those patients whose disease level has progressed to an advanced stage. The results from this classification were compared against the rheumatologists' evaluation. It is important to note that the rheumatologists evaluation of the patients used here was as at the time of the imaging session and might not necessarily reflect those patients disease activity at this present time.

Table 4-13: Min and Max dataset Decision Tree result for level of severity (Using 26 patients and 26 control subjects)*

\begin{tabular}{|c|l|r|r|r|r|r|}
\hline \multicolumn{1}{|c|}{ DT\# } & $\mathbf{1}$ & $\mathbf{2}$ & $\mathbf{3}$ & $\mathbf{4}$ & $\mathbf{5}$ \\
\hline \multirow{3}{*}{ Train } & Sensitivity & 100 & 100 & 88.2 & 94.4 & 94.4 \\
& Specificity & 90.1 & 90.1 & 83.7 & 80.2 & 80.2 \\
& CCR & 90.3 & 90.3 & 81.1 & 82.5 & 82.5 \\
\hline \multirow{3}{*}{ Test } & Sensitivity & 94.1 & 86.9 & 94.1 & 92.2 & 92.2 \\
& Specificity & 82.1 & 76.5 & 73.3 & 88.1 & 78.7 \\
& CCR & 80.0 & 77.7 & 73.3 & 89.2 & 78.5 \\
\hline
\end{tabular}


The result (Table 4-13) of the DT using the Min and Max dataset showed the highest level of consistent accuracy in terms of its specificity and sensitivity among the five bootstrapped DT's of the dataset combinations used. The variables that varied in the DT's were the AGE, YEARS, the joints (KNEE, ANKLE, MCP2, and MCP3), and MOB (as described in Table 3-6). The root variable which was the Min and Max temperatures remained the same among the five bootstrapped DT's; this was used to create all five bootstrapped DT's. The AGE, KNEE and ANKLE, YEARS were used to create DT 2 and 3, while the MCP2, MCP3, MOB were used to create DT 4 and 5. DT \#4 had the highest CCR at $89.2 \%$ with a sensitivity of $92 \%$ and a sensitivity of $88 \%$. This same step was repeated for the rest of the dataset combinations and it was established that the Min and Max dataset had the highest sensitivity and specificity of the datasets. The results for the DT's are shown in the table below (Table 4-14). This table illustrates the efficiency/accuracy of the classifiers and then compares them against one another. The Min/Max and Median/Max; Median/Max and Mode/Min; Min/Max and Median/Min; and Min/Max and Mode/Min also proved to be good discriminators with a sensitivity that ranged from $85-92 \%$ and a specificity that ranged from $80-88 \%$.

Table 4-14: Table showing the results of the DT combination of 12 different dataset series for the level of severity (Using 26 patients and 26 control subjects)

\begin{tabular}{|l|l|l|}
\hline Dataset & Sensitivity (\%) & Specificity (\%) \\
\hline Min and Max & 92 & 88 \\
\hline Min/Max and Median/Max & 85 & 80 \\
\hline Mode/Min and Median/Min & 77 & 70 \\
\hline Mode/Min and Mean/Min & 68 & 58 \\
\hline Mode/Max and Median/Max & 79 & 74 \\
\hline Min/Max and Median/Min & 90 & 85 \\
\hline Min/Max and Mean/Min & 65 & 60 \\
\hline
\end{tabular}




\begin{tabular}{|l|l|l|}
\hline Median/Max and Mode/Min & 88 & 82 \\
\hline Mean/Max and Min/Max & 76 & 73 \\
\hline Min/Max and Mode/Min & 86 & 80 \\
\hline Mode/Min and Median/Max & 55 & 45 \\
\hline Mode/Max and Mean/Max & 56 & 60 \\
\hline
\end{tabular}

The results from the classifier were compared to the evaluations of the rheumatologist to determine the accuracy of the classifier in separating the patients into these three classes. The table below (Table 4- 15) illustrates this. The rheumatologist's evaluation of patients 1 through 13 was adapted from previous work (Adéa 2009) from his evaluation of those patients at the time of the imaging session. So it is important to note that though the disease status of those patients might currently not be the same as those evaluations made, as of the time of imaging, these were their level of RA activity. Patients 14 through 26 were the evaluations for the newly recruited patients that he did as of the time of their imaging status (Appendix F for a copy of his official evaluation).

Table 4-15: Table showing the results of the classifier and the rheumatologists' evaluation

\begin{tabular}{|c|c|c|c|}
\hline $\begin{array}{c}\text { Patient } \\
\#\end{array}$ & Sex & $\begin{array}{c}\text { Rheumatologist's } \\
\text { Evaluation }\end{array}$ & $\begin{array}{c}\text { See5 } \\
\text { Classification }\end{array}$ \\
\hline 1 & M & Low & Low \\
\hline 2 & F & Low & Low \\
\hline 3 & M & High & Medium \\
\hline 4 & F & Low & Low \\
\hline 5 & F & Low & Low \\
\hline 6 & M & Low & Medium \\
\hline 7 & F & High & High \\
\hline 8 & F & Low & Low \\
\hline 9 & M & Low & Low \\
\hline 10 & F & Medium & Low \\
\hline 11 & F & Medium & Medium \\
\hline
\end{tabular}




\begin{tabular}{|c|c|c|c|}
12 & $\mathrm{~F}$ & Low & Low \\
\hline 13 & $\mathrm{~F}$ & Medium & Medium \\
\hline 14 & $\mathrm{~F}$ & Medium & High \\
\hline 15 & $\mathrm{~F}$ & Low to Medium & Low \\
\hline 16 & $\mathrm{M}$ & Low to Medium & Medium \\
\hline 17 & $\mathrm{~F}$ & Low & Low \\
\hline 18 & M & Low to Zero & Low \\
\hline 19 & $\mathrm{~F}$ & Low to Zero & Low \\
\hline 20 & M & Low to Medium & Medium \\
\hline 21 & $\mathrm{~F}$ & Low to Zero & Low \\
\hline 22 & $\mathrm{~F}$ & Low & Low \\
\hline 23 & F & Medium to High & High \\
\hline 24 & F & Low to Zero & Low \\
\hline 25 & F & Medium & Medium \\
\hline 26 & F & Low to Zero & Low \\
\hline
\end{tabular}

Table 4-15 displays the assessment by the physician and the classification by DT, at this stage it is difficult to conclude that one is more accurate than the other, as physical assessment by physicians can still be problematic. However, since many of the patient cases were rated similarly by both methods (physician and DTs), it is encouraging and with more accurate development of our infrared approach and analyses, we may be able in the near future to create a useful tool to measure a patient's current status, and more particularly, the progress or regress that a patient goes through with this disease.

Table 4-16 shows the contingency table for the classifier when using the Min and Max dataset as inputs into the classifier with a combination of the knee, ankle, $2^{\text {nd }} \mathrm{MCP}$ and $3^{\text {rd }} \mathrm{MCP}$ as the joints of interest. From this table, the number of true positives were 90 , where the classifier correctly classified, the number of variances from the physician for what would be called "false negatives" were 8, where the classifier classified a patient at MEDIUM as LOW or at HIGH as MEDIUM; the number of "false positives" were 2, where the classifier classified a 
patient at LOW as MEDIUM or at MEDIUM as HIGH, and the number of "true negatives" were 13, where the classifier classified a patient as MEDIUM, where the patient could either be classified as LOW or MEDIUM, or HIGH, where the patient could be classified as MEDIUM or HIGH.

Table 4-16: Contingency table for Min and Max dataset using the knee, ankle, $2^{\text {nd }}$ MCP and $3^{\text {rd }}$ MCP

\begin{tabular}{|c|c|}
\hline 162 & 16 \\
\hline 4 & 26 \\
\hline
\end{tabular}

Table 4-17 shows the confusion matrix for the dataset of the three classes: LOW, MEDIUM and HIGH classification of the level of RA severity in the patient group, using the Min and Max temperature measurements of the knee, ankle, $2^{\text {nd }}$ MCP and $3^{\text {rd }}$ MCP. This illustrates the accuracy of the classifier in these three levels.

Table 4-17: Confusion matrix for the Min and Max dataset

\begin{tabular}{|c|c|c|c|c|c|}
\hline & \multicolumn{5}{|c|}{ PREDICTED } \\
\hline \multirow[t]{5}{*}{ ACTUAL } & & LOW & MEDIUM & HIGH & $\overline{\text { SUM }}$ \\
\hline & LOW & 128 & 16 & 0 & 144 \\
\hline & MEDIUM & 14 & 38 & 6 & 58 \\
\hline & HIGH & 0 & 2 & 4 & 6 \\
\hline & SUM & 142 & 56 & 10 & 208 \\
\hline
\end{tabular}


From these results, the following conclusions were made; hence satisfying the stated objective:

1- The classifier was able to determine the level of RA activity in the patient group and was able to separate them into LOW, MEDIUM and HIGH.

2- The best dataset combination that effectively determined in presence or absence of RA from the sample were the Min and Max; Min/Max and Median/Max; Min/Max and Median/Min; Median/Max and Mode/Min; and Min/Max and Mode/Min.

\subsection{Discussion}

All the objectives stated were successfully accomplished. The first objective which was to analyze the joints to show which joint could statistically discriminate between the patient and control groups was successfully accomplished. From previous work done in 2009, Adea found that the Knee, $2^{\text {nd }} \mathrm{MCP}$ and $3^{\text {rd }} \mathrm{MCP}$ statistically discriminated between the control and patient groups; however the scope of her analysis was limited to the joints in the hands and Knees. In this thesis, the rest of the joints (Elbows, Ankles and Feet) were analyzed, and from this analysis, it was established that the ankles were also able to statistically discriminate between the patients and control subjects. These statistical analyses were carried out using MATLAB's Lilliefors test, Kruskal-Wallis test and Ranksum test. The results for the lilliefor and ranksum tests only were included in this thesis so as to avoid redundancy, because the kruskal-Wallis test was run as a confirmatory test. 
The second objective which was using machine learning (Decision Trees) to generate a classifier that could show the presence or absence of RA, and to also show whether an increase in the sample size improved or deteriorated the effectiveness of the classifier were also successfully accomplished. From the results, it was deduced that not only was the classifier able to discriminate between the patients and control subjects with specificity and sensitivity of 94 and $96 \%$ respectively, but it also showed that the recruitment of additional participants (13 patients and 16 control subjects) into the study also helped to increase the performance of the classifier ( $9 \%$ in the sensitivity and $3 \%$ increase in the specificity).

The third objective which was to determine which joints were the best indicators of RA was successfully accomplished. The classifier was able to show that the combination of either the Knee, $2^{\text {nd }} \mathrm{MCP}$ and $3^{\text {rd }} \mathrm{MCP}$ or the ankle, $2^{\text {nd }} \mathrm{MCP}$ and $3^{\text {rd }} \mathrm{MCP}$ were the best indicators of RA. The Min and Max dataset also proved to be the best discriminators with a sensitivity and specificity of 95 and $91 \%$ respectively. However to further establish which joints were the best indicators of RA, from the four joints that were statistically proven to discriminate between the patients and the control subjects, these joints were separated and then individually inputted into the DT. The $3^{\text {rd }} \mathrm{MCP}$ and $2^{\text {nd }} \mathrm{MCP}$ proved to be the joints that best indicated the presence of RA in the patients.

The fourth and last objective, which was to determine the level of RA activity in the patient group and separate them into three classes, LOW, MEDIUM and HIGH was successfully accomplished. The Min and Max dataset proved to be the best dataset in properly classifying the group into these three classes. The results of the DT, establishing the accuracy and efficiency of the classifier were confirmed by the rheumatologist's evaluations of the patients. These results were encouraging because they showed some consistency with the rheumatologist's evaluations. 


\section{CHAPTER FIVE}

\section{CONCLUSIONS}

This chapter provides the conclusion of this work and contributes new knowledge, to the previous work done. It also proposes some new research questions for future works to be done in the advancement of this study.

\subsection{Conclusions}

The four objectives that were stated at the beginning of this thesis were all accomplished. Hence the following conclusions were made from the body of the work.

1. It was observed that the 1st MTP was able to differentiate between the patients and the control subjects, however after further literature review, it was concluded that this difference could possibly be attributed to osteoarthritis and not RA, because the former is the common aging of the bones which affects the general population at one point or another as they age. This statistical analysis was done by using the non-parametric ranksum and kruskal-Wallis tests. These joints statistically showed at a significance level of $5 \%$ that they were able to differentiate between the control's datasets measurements and the patient's dataset measurements.

2. After successfully separating patients and control subjects, it was determined that, even though a couple of dataset series: Min and Max, Min/Max and Median/Max, Mode/Min and Median/Min, Min/Max and Median/Min, and Mode/Max and Mean/Max, gave high specificity and sensitivity, and acceptable discrimination parameters, the Min and Max temperature series gave the highest specificity at $92 \%$ and sensitivity at $96 \%$. 
3. The Min and Max dataset consistently proved to be the best dataset among all the datasets; it showed the highest sensitivity and specificity throughout this thesis.

4. The third objective which was to determine which specific joints were best indicators of RA in terms of classification. This was accomplished in two ways, by the process of elimination of variable (joints), where a combination of joints were inputted into the decision tree to establish which joints were good indicators. From this the observation was made that, where the $2^{\text {nd }}$ and $3^{\text {rd }}$ MCP's were present, there was an improvement to the accuracy of the classifier generated; the conclusion was then made that the $2^{\text {nd }}$ and $3^{\text {rd }}$ MCP's were the best joints for the classification. This was further confirmed a second way, where the joint were separated and generated individual classifiers, the results confirmed the previously made assertion. The $2^{\text {nd }} \mathrm{MCP}$ had a specificity of $86 \%$ and a sensitivity of $80 \%$, while the $3^{\text {rd }} \mathrm{MCP}$ had a specificity of $90 \%$ and a sensitivity of $88 \%$.

From this work and previous work, TIR imaging has been used to determine the presence of RA in the smaller joints of the hands, the $2^{\text {nd }}$ and $3^{\text {rd }}$ MCP's, where RA is known to develop in its early onset. This can also help clinicians assess the presence of RA early on by focusing on those joints that have been shown to be good indicators of the presence of RA. It can also help them better manage those patients that are already diagnosed with RA effectively and efficiently.

\subsection{Contribution to knowledge}

The first contribution to knowledge was the completion of the first objective of the thesis, completing the statistical analysis of the joints that were not analysed in the previous work (by Adéa) to determine if there were other joints other than the $2^{\text {nd }} \mathrm{MCP}, 3^{\text {rd }} \mathrm{MCP}$ and Knees that would be indicators of RA and could also differentiate between the patients and control subjects. 
From the statistical analysis done on the elbows, feet and ankles (in this thesis), it was observed that the ankles are also indicators of RA.

The second contribution which was the main purpose of this thesis in the advancement of this study was the successful classification of the patients and control subjects. Using various combinations of the datasets, it was observed that the decision tree could successfully discriminate between the patients and control subjects. This new knowledge is particularly significant because it sheds new light on an area of analysis of TIR images that had not been explored in previous works. This helps to further solidify the status of TIR imaging of joints of RA patients as a potential tool that could help assess (1) the joints that need focused therapy in individual patients and (2) could help rheumatologists in early diagnosis or management therapy of already diagnosed patients in cases where physical examinations have limited usefulness.

The new knowledge gathered from this study has further buttressed the findings from the previous work (Adéa 2009) done, where from the statistical tests, it was determined that the Knee, $2^{\text {nd }} \mathrm{MCP}$ and $3^{\text {rd }} \mathrm{MCP}$ were the best joints that could discriminate between the patients and control groups. From the results of the classifier, while satisfying second objective, it was concluded that the same joints that were proven to be the best in determining the presence of RA statistically, were also the proven to be good indicators of RA (the combination of the Knee, $2^{\text {nd }}$ $\mathrm{MCP}$ and $3^{\text {rd }} \mathrm{MCP}$ ) and then it was shown that individually in a classifier, the $3^{\text {rd }} \mathrm{MCP}$ had the highest sensitivity and specificity at indicating the presence of RA and also the $2^{\text {nd }}$ MCP. This new knowledge helps to reinforce TIR imaging as a promising early detection tool, as with the characteristic of RA, where during the early stages, it affects smaller joints commonly in the hands and feet and then progresses to larger joints in the knees and elbows. 


\subsection{Future work}

In order to further the thermal infrared imaging work of the MIRG's research team, it would be necessary to collect data on more patients to increase the sample size of the database to look at the impact it has on the classifier, whether it improves it or shows no impact. It would also be important to have a more diverse population during recruitment; all the patients diagnosed with RA were of Caucasian background, while about $40 \%$ of the control subjects were of Black ethnicity. From the measurements taken from the TIR images, it is important that note that, those control subjects of black ethnicity had slightly higher temperatures than those of Caucasian and Asian descent. From this observation, in future recruitment of patients, it would be important to also include patients of black ethnicity in the study to observe the effects race plays on the disease activity. As was also shown in this thesis, the recruitment of more patients in the study helped improve the classification; it showed a $9 \%$ improvement in the sensitivity and $3 \%$ improvement in the specificity. This would be an essential component in the furthering of this study.

The next step would be to explore more image processing techniques, to allow better processing and analysis of the images. The incorporation of automated segmentation and automated extraction of ROI would allow this to be possible. Currently the ROI's are being selected manually; this limits the analysis due to subjectivity from the interpreter.

The next step would be to further investigate the statistical tests performed on the datasets of these joints to further validate their accuracy. Due to the size of the datasets and the various variables being tested, these datasets are susceptible to type- 1 errors. This type of error occurs when the null hypothesis is rejected when it is in fact true. 
The next step would be to further analyze the joints to establish if the rest of the joints of the feet and elbow, which were unable to discriminate between the patient and control subjects, could discriminate between the two groups using supervised learning and to further investigate other methods of improving the assessment and analysis of the TIR images. Another classification method that would be worth exploring would be fuzzy classifiers, and compare the results to the current method of classification used in this thesis. Other classifiers to be considered would be artificial neural network (ANN) models.

The next step then would be the correlation of IR imaging results, statistical and classification, to be compared to RA diagnosis gold standards such as MRI and HRUS, which are the tools currently used to correctly diagnose RA. These tools can correctly classify the normal and RA patients. This would further solidify the conclusions stated in this thesis, that IR imaging is a promising tool that can be used to replace or used in support of MRI's and HRU's. This comparison would also assess if IR technology can detect inflammation as early on in the disease as MRI and HRUS. As discussed in the introductory chapter, detecting inflammation early would assist in diagnosing RA before the manifestation of clinical symptoms and improve the prognosis of the patient. 


\section{CHAPTER SIX}

\section{REFERENCES}

1. Abdel-Nasser AM, Rasker JJ and Valkenburg HA (1997) "Epidemiological and Clinical aspects relating to the variability of rheumatoid arthritis". Seminars in Arthritis \& Rheumatism 27:123-140

2. Adéa C (2009) "Severity of illness measures of Rheumatoid Arthritis using Thermal Infrared imaging. MASc Thesis, Department of systems and computer engineering, Carleton University.

3. Altman D and Bland A (1994) "Diagnostic Tests 2: Predictive power". British Medical Journal 309: 6947

4. Amalu WC (2003) “A review of Breast Thermography”. International Academy of Clinical Thermology.

5. Ambrosius W (2007) "Topics in Biostatistics". Methods in Molecular Biology. Humana Press $1^{\text {st }}$ Ed.

6. Anbar M (1991) “Objective assessment of clinical computerized thermal images". In Medical Imaging V: Image processing. Proceedings of SPIE 1445: 479 - 484.

7. Annett FC (1998) "The American Rheumatism association 1987 revised criteria for the classification of rheumatoid Arthritis". Annals of Rheumatic Diseases 31: 315 - 324

8. Bacon PA (1976) "Thermography in the assessment of inflammatory arthritis". Clinical Rheumatic Disease 2: $51-65$

9. Bird HA, Ring EFJ, and Bacon PA (1979) "A thermographic and clinical comparison of three intra-articular steroid preparations in rheumatoid arthritis". Annals of the Rheumatic Diseases 28: 36 - 39

10. Chopra A and Abdel-Nasser A (2008) "Epidemiology of rheumatic musculoskeletal disorders in the developing world". Best Practice \& Research Clinical Rheumatology 22:583-604

11. Collins AJ and Cosh JA (1970) "Temperature and biochemical studies of joint inflammation". Annals of the Rheumatic Diseases 29: 386 
12. Collins AJ, Ring EFJ, Cosh JA, and Bacon PA (1974) "Quantitation of thermography in arthritis using multi-isothermal analysis". Annals of Rheumatoid Disease 33: 113 - 115

13. Costenbader KH, Kang JH, and Karlson EW (2010) "Antioxidant intake and risks of rheumatoid arthritis and systemic lupus erythematosus in women". American Journal of Epidemiology 172:205-216.

14. Dash M and Liu H (1997) “Feature selection for classification". Intelligent Data Analysis. Elsevier, $131-156$

15. Davison AC and Kuonen D (2006) "Topics in statistical computing - An introduction to the bootstrap with applications in R". Statistical Computing and Statistical Graphics Newsletter 13: 6-11

16. Deveraux M (1985) "Disease activity indexes in rheumatoid arthritis: A prospective comparative study with thermography". Annals of the Rheumatic Diseases 44: 434 - 437

17. Diakides NA (2000) "The growing applications of medical infrared imaging". IEEE Engineering in Medicine and Biology 19: $28-29$

18. Duda R, Hart P, and Stork D (2001) "Pattern Classification". John Wiley and sons, Inc., $2^{\text {nd }}$ Ed. New York, NY

19. Efron B and Tibshirani RJ (1993) "A introduction to the bootstrap". Chapman and Hall, London, England

20. Fauci MA, Breiter R, Cabanski W, Fick W, Koch R, Ziegler J, and Gunapala SD (2001) "Medical Infrared imaging - differentiating facts from fiction, and the impact of high precision quantum well infrared photodetector camera systems and other factors in its reemergence". Infrared Physics And Technology 42: 337 - 344

21. Fawcett T (2006) “An introduction to ROC analysis". Pattern Recognition Letters. Elsevier, $867-871$

22. FLIR Systems (2007) ThermaCAM Researcher User's manual. Sweden: Publ. No. T559012 Rev. a249.

23. Frize M, Adéa $C$, Karsh J, Payeur $P$ "Detection of the Presence of Rheumatoid Arthritis Using Infrared Thermal Imaging". Accepted for presentation, SPIE Medical Imaging conference. Lake Buena Vista, Florida, Feb 2011

24. Frize M, Karsh J, Herry CL, Adéa C, Aleem I, Payeur P (2009) "Preliminary Results of Severity of Illness Measures of Rheumatoid Arthritis Using Infrared Imaging". Proc. MeMeA (Medical Measurements and Applications). Cetraro, Italy, May: 187-192. 
25. Galvin EM, Niehof Sjoerd M, Medina HJ., Zijlstra FJ., Bommel JV, Klein J, and Verbrugge SJC (2006) "Thermographic temperature measurement compared with pinprick and cold sensation in predicting the effectiveness of regional blocks". Anesthesia and Analgesia 102: $598-604$

26. Geurts P, Irrthum A and Wehenkel L (2009) "Supervised learning with decision treebased methods in computational and systems biology". Molecular Biosystems 5:15931605.

27. Goodman PH, Murphy MG, Siltanen GL, Kelly MP, and Rucker L (1986) “Normal temperature asymmetry of the back and extremities by computer assisted Infrared imaging". Thermology 1: 195 - 202

28. Herry CL, Frize M, and Goubran RA (2008) "Search for abnormal thermal patterns in clinical thermal infrared imaging". IEEE International workshop on medical measurements and applications $61-65$

29. Hilden J (1991) "The area under the ROC curve and its competitors". Medical Decision Making 11: 95 - 101

30. Hooshmand H, Hashmi M, and Phillips EM (2001) "Infrared thermal imaging as a tool in pain management - an 11 year study part I of II". Thermology Intermation 11: 53 - 65

31. Horvath SM, and Hollander JL (1949) "Intra-articular temperature as a measure of joint reaction". Journal of Clinical Investigation 28: $469-473$

32. Houdas $Y$ and Ring EFJ (1982) "Human body temperature: its measurement and regulation". Plenum Press

33. Ishii N, Koike A, Yamamoto $Y$ and Takagi $T$ (2010) "Figure classification in biomedical literature to elucidate disease mechanisms based on pathways". Artificial Intelligence in Medicine 117:

34. Kalla AA and Tikly M (2003) "Rheumatoid arthritis in the developing world". Best Practice \& Research Clinical Rheumatology 17: 863-875

35. Kelly H, Bull A, and McBryde E (2008) "Estimating sensitivity and specificity from positive predictive value, negative predictive value and prevalence: Application to surveillance system for hospital-acquired infections". Elsevier, 164 - 168

36. Kochi Y, Suzuki A, Yamada R and Yamamoto K (2009) "Genetics of rheumatoid arthritis: Underlying evidence of ethnic differences". Journal of Autoimmunity 32:158162 
37. Lyons BR and Orlove GL (2003) "A brief history of 25 years (or more) of Infrared imaging radiometers". Proceedings of SPIE 5073: $17-30$

38. Marra CA, Woolcott JC, Kopec JA, Shojania K, Offer R, Brazier JE, Esdaile JM and Anis AH (2005) "A comparison of generic, indirect utility measures (the HU12,HU13, SF-6D, and the EQ-5D) and disease specific instruments (the RAQoL and HAQ) in rheumatoid arthritis". Social Science \& Medicine 60:1571-1582

39. MATLAB R2009a. Software - help files. Version 7.8.0.347, Mathworks 2009

40. McIver DK and Friedl MA (2002) "Using prior probabilities in decision tree classification of remotely sensed data". Remote Sensing of Environment 81: 253 - 261

41. Mitra S and Acharya T (2003) Data mining multimedia soft computing and bioinformatics. John Wiley and Sons, Inc., Hoboken, NJ

42. Mody GM and Cardiel MH (2008) "Challenges in the management of rheumatoid arthritis in developing countries". Best Practice \& Research Clinical Rheumatology 22:621-641

43. Mooney C, Duval R, and Lewis-Beck M (1993) "Bootstrapping: A nonparametric approach to statistical inference". Sage Publications, Inc.

44. Ng EYK (2009) "A review of thermography as promising non-invasive detection modality for breast tumour". International Journal of Thermal Sciences 48:849-859.

45. Obuchowski N (2003) "Receiver operating characteristic curve and their use in radiology". Radiology 229: $3-8$

46. Phillips TJ (2002) "High performance thermal imaging technology". The Advanced Semiconductor Magazine 15: $32-34$

47. Prince J, and Links J (2006) "Medical imaging signals and systems". Pearson Education. Upper Saddle River, NJ.

48. Putley EH (1982) "History of infrared detection - Part II: The first thermographs". Infrared Physics 22: 189 - 191

49. Reinbery A (1976) "Circadian changes in the temperature of human beings". Clinical Rheumatic Disease 2: 128 - 139

50. Ring EFJ and Collins AJ (1970) "Quantitative thermography". Rheum Phys Med 10: 337

51. Ring EFJ, Collins AJ, Bacon PA, and Cosh JA (1974) "Quantitation of thermography in arthritis Using multi-isothermal analysis II". Ann Rheum Dis 33: 353 
52. Salisbury R (1983) "Heat distribution over normal and abnormal joints: thermal pattern and quantification". Annals of the Rheumatic Diseases 42: $494-499$

53. Salvatore D and Reagle D (2002) "Theory and problems of statistics and Econometrics" $2^{\text {nd }}$ Ed. Schaum's Outline series of McGraw-Hill Companies

54. Symmons D, Mathers C and Pfleger B (2006) "The global burden of rheumatoid arthritis in the year 2000"

55. Symmons DPM (2002) "Epidemiology of rheumatoid arthritis: Determinants of onset, persistence and outcome”. Best Practice \& Clinical Research Rheumatology 16: 702-722

56. Tan P, Steinbach and Kumar V (2006) "Introduction to data mining". Pearson Education Inc., Boston MA

57. Toban GJ, Youinou P and Saraux A (2010) "The environment, geo-epidemiology and autoimmune disease: Rheumatoid arthritis". Journal of Autoimmunity 35:10-14.

58. Uematsu S, Edwin DH, Jankel WR, Kozikowski J, and Trattner M (1988) “Quantification of thermal asymmetry Part 1: Normal values and reproducibility". Journal of Neurosurgery 69: 552 - 555

59. Uhlig T, Fongen C, Steen E, Christie A and Odegard S (2010) "Exploring Tai Chi in Rheumatoid arthritis: a quantitative and qualitative study". BMC Musculoskeletal Disorders 11:43- 50 .

60. Unay D (2006) "Multispectral image processing and pattern recognition techniques for quality inspection of apple fruits" www.tcts.fpms.ac.be/publications/phds/unay/unay_thesis.pdf

61. Visser H (2005) "Early diagnosis of rheumatoid arthritis". Best Practice \& Research Clinical Rheumatology 19:55-72

62. Wang $X$ and Zhang JQ (2002) "Infrared Imaging System performance model based on the machine vision". Proceeding of SPIE 4925: 539 - 546

63. Woolf AD and Pfleger B (2003) "Burden of major musculoskeletal condition". Bulletin of the World Health Organization 81: 646- 657 


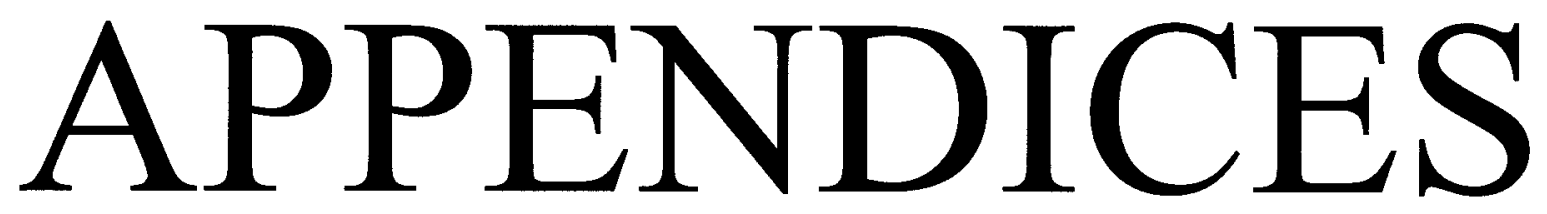




\section{APPENDIX A - Research Participant Questionnaire}

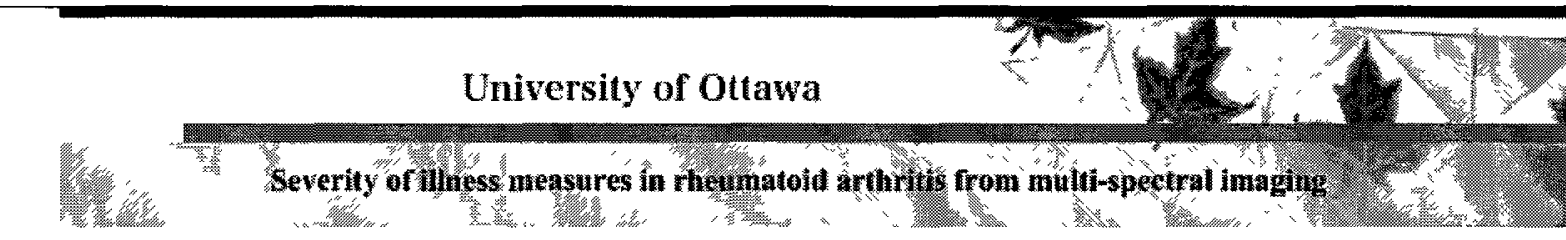

\section{Research Participant Questionnaire}

Name:

Email:

Telephone:

Sex:

Age:

Race:

Physical Medical disabilities/ Joint issues (If any):

Level of severity (of Pain) on a scale of $1-10$ (Personal Assessment)

- 1-4-LOW:

- 4-7-MEDIUM:

- 7-10- HIGH: 


\section{APPENDIX B - Research Participation Package}

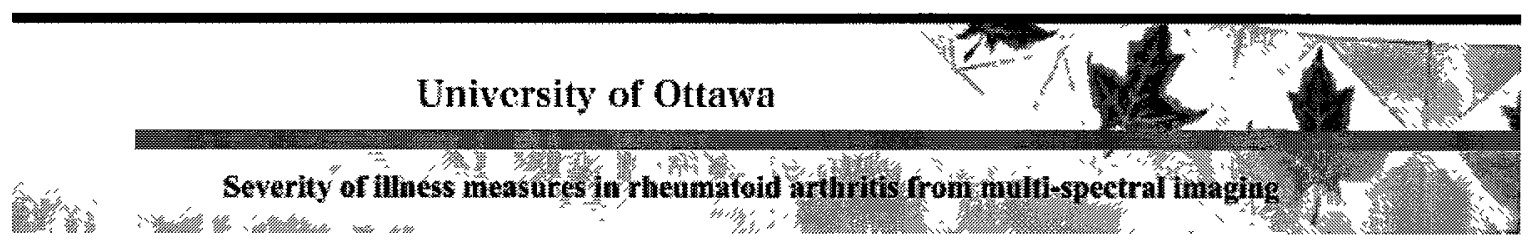

Research Participant Information and Consent Form

You are being asked to participate in a research study using an infrared (IR) camera (heat images) to determine temperature measurements of limbs. In addition, a series of color pictures using a digital camera will be taken to assess the level of mobility of extremities and joints. The research will be conducted at the University of Ottawa and will be scheduled at a convenient time.

The information collected will not contain pictures of the face and will be kept anonymous.

\section{Purpose and Design}

Rheumatoid arthritis affects 300,000 Canadians each year; it involves inflammation and symptoms like redness, swelling, heat, and pain. In this study, we plan to measure the temperature of the joints of patients diagnosed with rheumatoid arthritis and of participants without rheumatoid arthritis using an infrared camera. The assumption is that the infrared pictures of the rheumatoid arthritis patients will show inflammation of tissue or joints. A second test to be conducted at the same time is the taking of video pictures with digital cameras to assess the extent of movement of extremities and joints of both groups: patients with rheumatoid arthritis and healthy participants. This is a pilot project to assess whether these two types of tests can be helpful as an aid to the early diagnosis of rheumatoid arthritis. These tests may also help to monitor therapy in joint debilitating diseases.

Approximately 15 patients will be involved in this pilot study and 15 normal subjects.

Because the skin surface temperature variations are extremely subtle and sensitive to environmental changes:

- Do not use talcum powder, lotion, drug or deodorant on the skin on the day of the session.

- Do not consume alcoholic beverages 12 hours prior to the session.

- Do not consume hot beverages at least 1 hour prior to the session.

- Do not use procedures such as acupuncture, transcutaneous electrical stimulation (TENS machine), hot or cold patches, or any other form of physiotherapy at least $24 \mathrm{~h}$ prior to the session. You should not do any intense physical exercises at least 4 hours prior to the session

- Avoid prolonged sun exposure for at least one week prior to the session.

- Do not smoke at least 2 hours prior to the session.

- Do not wear any rings, necklaces, and bracelets during the session.

The session will take place in a room with the following characteristics: 
- Without windows or with covered windows to avoid any reflection.

- Constant uniform temperature between 20 and 25 .

- Constant humidity.

- Fluorescent lights.

You will be asked to come to the biomedical laboratory of Dr. Pierre Payeur at University of Ottawa (Colonel By, Room B407) at a mutually convenient time. Both types of cameras will be available at that location. You need to wear shorts and short sleeve shirt (or change into these in a nearby bathroom).

The skin has to cool at room temperature for 10-15 minutes; then pictures are taken of the joints, hands, feet. For each body region, you will be asked to assume a position facilitating the imaging. For instance, for images of the hands (palm and back), you will be seated on a chair and will be asked to place your hand over a table. For the under-feet view, you will be seated on a chair and raise your leg, with the ankle resting on a stool. For other views, you will be standing.

You will also be asked to move your hands, elbows, knees and feet from time to time. Sequences of images will be taken with an infrared camera and several regular intra-optical video cameras. Both types of cameras will record the sequences simultaneously.

The imaging session is expected to last approximately 30 minutes.

You may be asked to participate for a maximum of three sessions in order to study the stability of the temperature distribution over time. But this is not mandatory to participate in the study. It is totally voluntary.

\section{Are there any risks?}

There are no foreseen risks to participants.

If you are a patient diagnosed with rheumatoid arthritis, the research team will capture images of your body parts affected by rheumatoid arthritis.

If you are a healthy volunteer, the research team will capture images of several healthy joints and limbs.

There is no direct contact or risk associated with gathering these images.

Are there any benefits to me if I choose to participate? While there are no immediate benefits for you, this study is expected to provide a useful tool for early diagnosis of rheumatoid arthritis.

How will my confidentiality be protected? 
Your name will not be used in any publications that may arise from this study. Only group information will be published. No images of the participant's faces will be taken. The consent forms and images will be stored at Carleton and Ottawa Universities research laboratories of $\mathrm{Dr}$ Frize and Dr Payeur containing only numbers as reference. The images will be stored in a protected file with user id and password protection. The data will be destroyed five years after the tests are completed.

\section{Withdrawal from the Study}

If you are a patient with rheumatoid arthritis, you may withdraw from the study at any time without any impact to the care you are receiving. You can also choose to have your images withdrawn from the study completely.

If you are a healthy participant, you may withdraw from the study at any time. You can also choose to have your images withdrawn from the study completely.

\section{Study Expenses}

If you are a patient, you will be reimbursed for parking or bus expenses to attend the session at University of Ottawa. If you are a student, no compensation will be needed, as you will be on either campus and there is a free bus service between the two universities.

\section{Questions about the Study}

You may ask any questions about the research at any time. If you have questions about the research after you leave today you should contact the student co-investigator Abiola Ogungbemile at (613) $858-5712$ or (613) $520-2600$ ext 2068 or email: abiola.ogungbemile@gmail.com.

If you have any questions about your rights as a research subject, you may contact Leslie MacDonald-Hicks, Carleton University Research Ethics Board Coordinator at (613) 520-2517, email ethics@carleton.ca.

\section{Consent}

I have read this Research Participant Information and Consent Form and have had an opportunity to ask my study doctor and the researchers any questions I had about the study.

My questions and/or concerns have been answered to my satisfaction and I agree to participate in this study. If I decide at a later stage in the study that I would like to withdraw my consent, I may do so at any time.

A copy of the Research Participant Information and Consent Form will be provided to me.

\section{Signatures}


Name of Participant (please print):

Signature

Date

Student co-investigator: Abiola Ogungbemile

Signature

Date 


\section{APPENDIX C - FLIR Technical Specification}

\section{FLIR A320 Camera}

\section{IMAGING PERFORMANCE}

Field of view/min focus distance $25^{\circ} \times 18.8^{\circ}$ $10.4 \mathrm{~m}$

Spatial resolution (IFOV) $1.36 \mathrm{mrad}$

Thermal sensitivity/NETD @ 50mk @ +30 ㄷ

Focusing Automatic or manual (built in motor)

Detector type Focal Plane Array (FPA), uncooled microbolometer

Spectral range 7.5 to $13 \mu \mathrm{m}$

\section{IMAGE PRESENTATION}

Ethernet output IEEE-1394 8/16-bit monochrome and 8-bit color

Video output composite video output, PAL and NSTC compatible

\section{MEASUREMENT}

Temperature ranges

Range $1:-40^{\circ} \mathrm{C}$ to $+120^{\circ} \mathrm{C} \quad(-40$ to $+248^{\circ} \mathrm{F}$ )

Range 2: $0^{\circ} \mathrm{C}$ to $+500^{\circ} \mathrm{C} \quad(+32$ to $+932^{\circ} \mathrm{F}$ )

Optional: Up to $+1500^{\circ} \mathrm{C}\left(+2732^{\circ} \mathrm{F}\right)$

Optional: Up to $+2000^{\circ} \mathrm{C}\left(+3632^{\circ} \mathrm{F}\right)$

Accuracy (\% of reading) $\pm 2^{\circ} \mathrm{C}$ or $\pm 2 \%$

Measurement modes Spot, Area, Isotherm, Difference

Automatic emissivity correction Variable from 0.1 to 1.0

Individual emissivity settings Individually settable

Measurement corrections Reflected ambient, distance, relative humidity, external optics. Automatic, based on user input

\section{POWER SOURCE}

External power operation 12/24 VDC, 24W absolute max

External power, connector type 2 pole jackable screw terminal

Voltage allowed range $10-30$ VDC

\section{PHYSICAL CHARACTERISTICS}

Weight $0.7 \mathrm{~kg}(1.5 \mathrm{lbs})$

Size $170 \mathrm{~mm} \times 70 \mathrm{~mm} \times 70 \mathrm{~mm}$

Tripod mounting UNC 1/4"- 20 (on three sides)

\section{ENVIRONMENTAL}

Operating temperature range $-15^{\circ} \mathrm{C}$ to $+50^{\circ} \mathrm{C}$ $\left(5^{\circ} \mathrm{F}\right.$ to $\left.122^{\circ} \mathrm{F}\right)$

Storage temperature range $-40^{\circ} \mathrm{C}$ to $+70^{\circ} \mathrm{C}(-$ $40^{\circ} \mathrm{F}$ to $158^{\circ} \mathrm{F}$ )

Humidity Operating and storage $95 \%$ relative humidity $+25^{\circ} \mathrm{C}$ to $+40^{\circ} \mathrm{C}$

\section{Encapsulation}

IP (IEC 60529)

Shock Operational: 25G, IEC 60068-2-29

Vibration Operational: 2G, IEC 60068-2-6 


\section{APPENDIX D - Raw Measurements of the Joints}

10.1. $2^{\text {nd }} M C P$ Raw Data Measurements

Table 10-1: Raw Data Measurements Extracted from the Control Group's 2nd MCP Joint

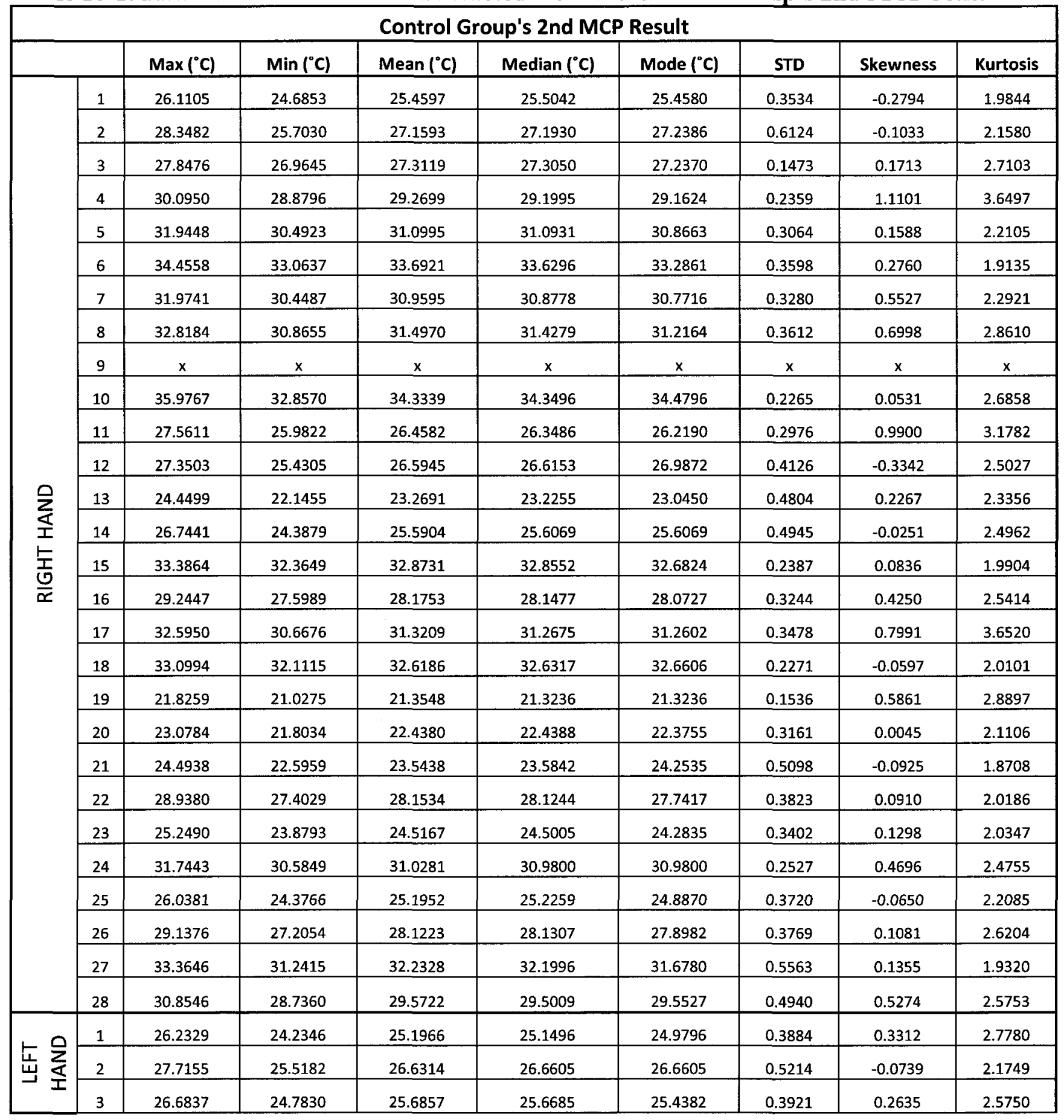




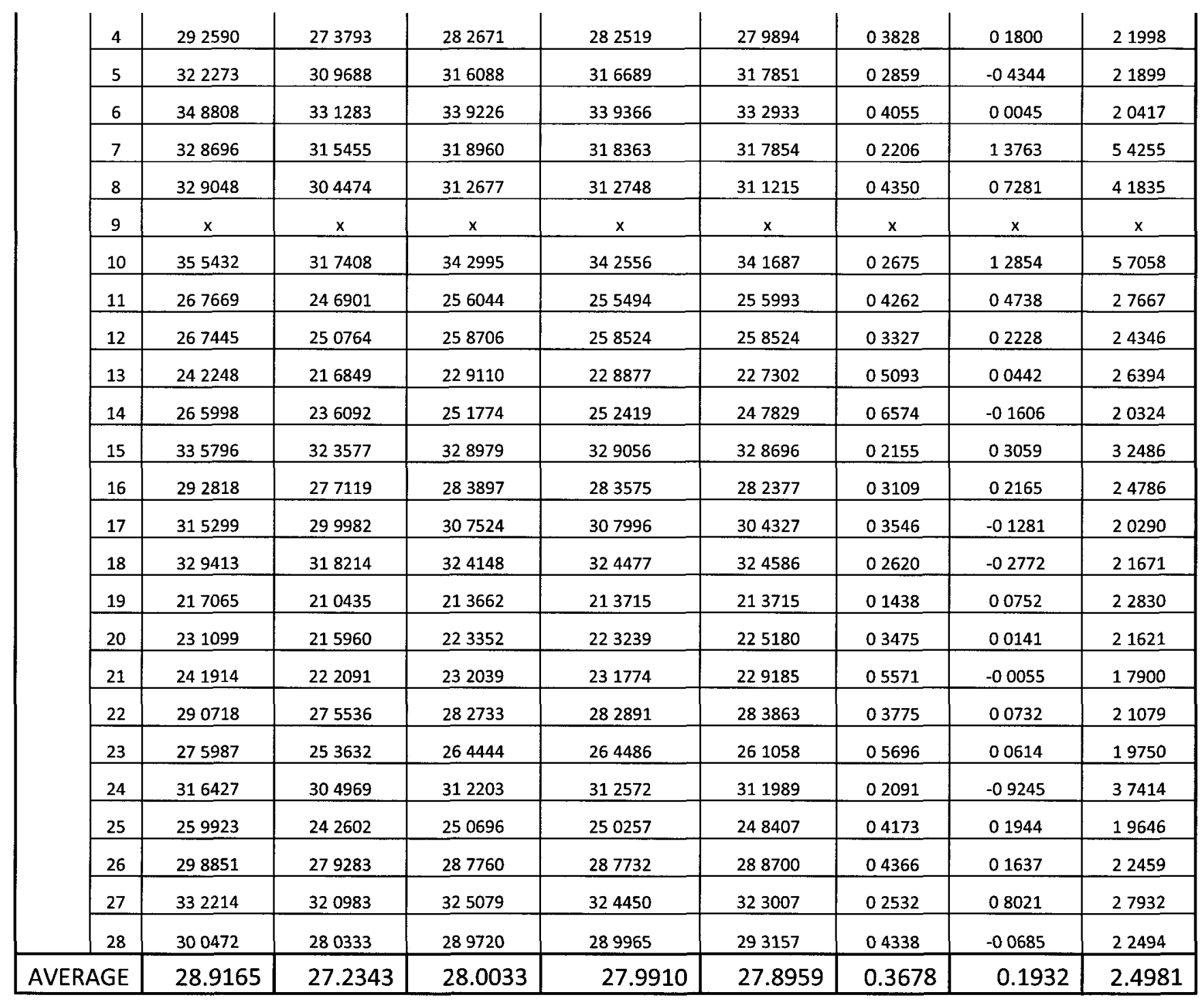




\subsection{2nd MCP Raw Data Measurements}

Table 10-2: Raw Data Measurements Extracted from the Patient Group's 2nd MCP Joint

\begin{tabular}{|c|c|c|c|c|c|c|c|c|c|}
\hline \multicolumn{10}{|c|}{ Patient Group's 2nd MCP Result } \\
\hline & & $\operatorname{Max}\left({ }^{\circ} \mathrm{C}\right)$ & $\operatorname{Min}\left({ }^{\circ} \mathrm{C}\right)$ & Mean $\left({ }^{\circ} \mathrm{C}\right)$ & Median ( $\left.{ }^{\circ} \mathrm{C}\right)$ & Mode $\left({ }^{\circ} \mathrm{C}\right)$ & STD & Skewness & Kurtosis \\
\hline \multirow{26}{*}{ 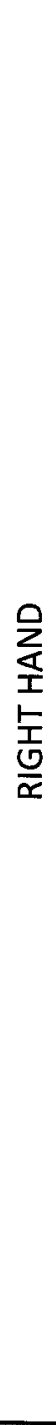 } & 1 & 34.1853 & 32.5157 & 32.9144 & 32.8760 & 32.7752 & 0.2123 & 1.8015 & 8.9345 \\
\hline & 2 & 34.3468 & 32.9070 & 33.5189 & 33.5539 & 33.7114 & 0.2631 & -0.0834 & 2.7726 \\
\hline & 3 & 33.6470 & 31.7626 & 32.7416 & 32.8710 & 33.0655 & 0.4647 & -0.2646 & 1.6601 \\
\hline & 4 & 30.0216 & 28.3053 & 29.0348 & 29.0589 & 29.3783 & 0.3849 & -0.0057 & 1.8287 \\
\hline & 5 & 29.8801 & 28.3182 & 29.2812 & 29.3025 & 29.2802 & 0.3420 & -0.4080 & 2.4527 \\
\hline & 6 & 31.6099 & 27.4611 & 29.1036 & 28.9679 & 28.8114 & 0.9284 & 0.4308 & 2.3475 \\
\hline & 7 & 34.1497 & 32.4363 & 33.2885 & 33.2890 & 33.2639 & 0.2014 & -0.0640 & 3.8788 \\
\hline & 8 & 32.9120 & 31.7341 & 32.2329 & 32.1835 & 32.0749 & 0.2439 & 0.4930 & 2.4673 \\
\hline & 9 & 31.2683 & 29.5413 & 30.2286 & 30.2426 & 30.1911 & 0.2889 & 0.0198 & 2.8278 \\
\hline & 10 & 33.9721 & 31.4287 & 32.4823 & 32.4658 & 32.7470 & 0.5197 & 0.2233 & 2.3592 \\
\hline & 11 & 33.7791 & 31.7989 & 32.5586 & 32.5878 & 32.8184 & 0.3941 & -0.0181 & 1.9991 \\
\hline & 12 & 32.6211 & 31.3234 & 31.8121 & 31.8036 & 31.8254 & 0.2546 & 0.3638 & 2.7489 \\
\hline & 13 & 28.5139 & 27.5378 & 28.0228 & 28.0569 & 28.1170 & 0.1676 & -0.5022 & 2.8177 \\
\hline & 14 & 36.3383 & 34.7329 & 35.3963 & 35.3733 & 35.2655 & 0.3504 & 0.2665 & 2.4103 \\
\hline & 15 & 33.5002 & 30.7022 & 31.0767 & 31.0617 & 30.9811 & 0.1701 & 0.4063 & 2.8042 \\
\hline & 16 & 34.9065 & 31.0051 & 32.8640 & 32.8372 & 32.6836 & 0.3564 & 0.4078 & 3.2602 \\
\hline & 17 & 34.4888 & 32.7380 & 33.2672 & 33.1863 & -66.0258 & 0.3474 & 0.9733 & 3.7471 \\
\hline & 18 & 36.0788 & 33.7222 & 34.6419 & 34.6090 & 34.2365 & 0.5284 & 0.3357 & 2.2699 \\
\hline & 19 & 33.8627 & 30.8673 & 33.3783 & 33.3936 & 33.4484 & 0.2509 & -0.0505 & 2.0165 \\
\hline & 20 & 29.5843 & 27.3403 & 28.3677 & 28.2695 & 27.6421 & 0.6476 & 0.3143 & 1.8257 \\
\hline & 21 & 32.7047 & 29.9366 & 30.4865 & 29.3718 & 30.1124 & 0.3896 & 0.9468 & 3.1642 \\
\hline & 22 & 32.1840 & 29.8371 & 30.7553 & 30.7145 & 30.0742 & 0.5716 & 0.3297 & 2.1158 \\
\hline & 23 & 31.9303 & 29.9401 & 30.4722 & 30.2607 & 29.9401 & 0.5333 & 1.1767 & 3.4005 \\
\hline & 24 & 35.7879 & 33.8939 & 34.5202 & 34.4695 & 34.3530 & 0.3566 & 0.6349 & 3.2274 \\
\hline & 25 & 26.0395 & 24.1037 & 24.9394 & 24.9486 & 24.5464 & 0.4471 & 0.1902 & 2.2609 \\
\hline & 26 & 26.1413 & 24.6106 & 25.2165 & 25.1823 & 24.8118 & 0.3880 & 0.3001 & 1.9468 \\
\hline \multirow{8}{*}{$\begin{array}{l}\text { 是 } \\
\text { 志 } \\
\text { 놇 }\end{array}$} & 1 & 33.3571 & 32.3134 & 32.7815 & 32.7824 & 32.7176 & 0.1961 & -0.0138 & 2.5599 \\
\hline & 2 & 33.7114 & 31.8063 & 33.2150 & 33.2453 & 33.5610 & 0.2907 & -0.9527 & 4.4021 \\
\hline & 3 & 34.1972 & 27.4902 & 33.4464 & 33.4965 & 33.6399 & 0.4492 & -5.7915 & 5.5579 \\
\hline & 4 & 30.1469 & 28.3801 & 29.5020 & 29.5117 & 29.5117 & 0.2609 & -0.3176 & 3.5379 \\
\hline & 5 & 29.3841 & 27.5893 & 28.5867 & 28.5875 & 28.4455 & 0.3419 & -0.0962 & 2.3800 \\
\hline & 6 & 28.5128 & 26.3002 & 27.2766 & 27.2760 & 26.9391 & 0.4515 & 0.1988 & 2.4525 \\
\hline & 7 & 33.6004 & 32.1542 & 33.0773 & 33.1060 & 33.0126 & 0.2997 & -0.7272 & 3.1715 \\
\hline & 8 & 33.0844 & 31.5378 & 32.3608 & 32.4148 & 32.5808 & 0.3553 & -0.1136 & 1.9768 \\
\hline
\end{tabular}




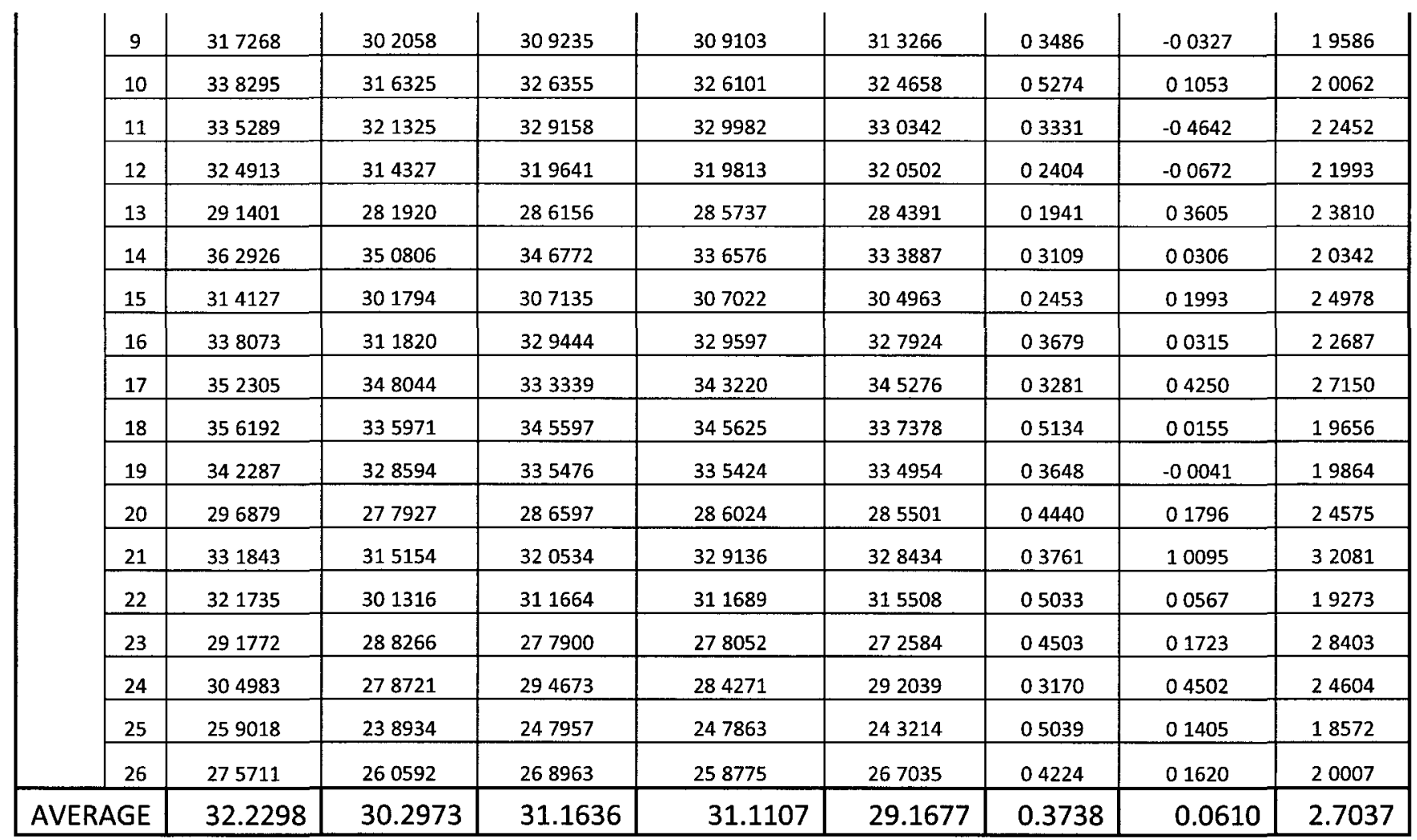




\section{3. $\quad 3^{\text {rd }}$ MCP Raw Data Measurements}

Table 10-3: Raw Data Measurements Extracted from the Control Group's $3^{\text {rd }}$ MCP Joint

\begin{tabular}{|c|c|c|c|c|c|c|c|c|c|}
\hline \multicolumn{10}{|c|}{ Control Group's 3rd MCP Result } \\
\hline & & $\operatorname{Max}\left({ }^{\circ} \mathrm{C}\right)$ & $\operatorname{Min}\left({ }^{\circ} \mathrm{C}\right)$ & Mean $\left({ }^{\circ} \mathrm{C}\right)$ & Median ( ${ }^{\circ} \mathrm{C}$ ) & Mode $\left({ }^{\circ} \mathrm{C}\right)$ & STD & Skewness & Kurtosis \\
\hline \multirow{28}{*}{$\begin{array}{l}\text { 足 } \\
\text { 至 } \\
\text { 皇 } \\
\frac{\mathbb{0}}{\simeq}\end{array}$} & 1 & 25.9880 & 24.4990 & 25.2549 & 25.2653 & 24.9099 & 0.3747 & 0.0264 & 2.0235 \\
\hline & 2 & 28.8054 & 27.2082 & 28.1365 & 28.1678 & 28.4833 & 0.3883 & -0.2086 & 1.9324 \\
\hline & 3 & 28.1704 & 27.0402 & 27.6344 & 27.6595 & 27.7423 & 0.2372 & -0.2217 & 2.2984 \\
\hline & 4 & 30.3895 & 29.2887 & 29.7749 & 29.7406 & 29.6297 & 0.2470 & 0.3682 & 2.2880 \\
\hline & 5 & 31.6907 & 30.3086 & 30.8597 & 30.8224 & 30.6758 & 0.3011 & 0.4344 & 2.3225 \\
\hline & 6 & 34.3919 & 32.4155 & 33.1733 & 33.0996 & 33.0206 & 0.4710 & 0.5141 & 2.5070 \\
\hline & 7 & 32.3649 & 30.7349 & 31.1827 & 31.1300 & 30.9985 & 0.2778 & 1.0317 & 4.2677 \\
\hline & 8 & 31.9513 & 30.6896 & 31.1362 & 31.1361 & 30.8435 & 0.2498 & 0.4963 & 2.7302 \\
\hline & 9 & $x$ & $x$ & $x$ & $x$ & $x$ & $x$ & $x$ & $x$ \\
\hline & 10 & 35.4865 & 31.8650 & 34.2666 & 34.1839 & 34.1332 & 0.2519 & 1.7503 & 6.5791 \\
\hline & 11 & 27.5762 & 26.8807 & 27.1890 & 27.1761 & 27.0172 & 0.1694 & 0.1757 & 2.0087 \\
\hline & 12 & 27.8551 & 26.3108 & 27.1695 & 27.1765 & 27.1538 & 0.2794 & -0.3697 & 3.2960 \\
\hline & 13 & 24.4112 & 22.8169 & 23.6351 & 23.6326 & 23.4762 & 0.3256 & -0.0919 & 2.3508 \\
\hline & 14 & 27.3121 & 25.8369 & 26.6115 & 26.6378 & 26.7897 & 0.3297 & -0.3488 & 2.3923 \\
\hline & 15 & 32.9127 & 32.2348 & 32.4359 & 32.4372 & 32.4516 & 0.1082 & 0.5938 & 3.7834 \\
\hline & 16 & 28.9249 & 27.2517 & 27.8189 & 27.7495 & 27.6140 & 0.3378 & 0.6234 & 2.6660 \\
\hline & 17 & 32.1180 & 30.5869 & 31.0833 & 31.0338 & 30.8875 & 0.2834 & 0.6766 & 2.9600 \\
\hline & 18 & 33.1855 & 32.1260 & 32.5662 & 32.5380 & 32.3719 & 0.2448 & 0.3700 & 2.2187 \\
\hline & 19 & 30.1383 & 28.1260 & 28.4923 & 28.4509 & 28.3396 & 0.2485 & 0.8548 & 3.5078 \\
\hline & 20 & 27.7442 & 25.1941 & 26.8322 & 25.7341 & 26.2961 & 0.1957 & 0.7984 & 3.1826 \\
\hline & 21 & 26.3565 & 24.4733 & 25.2942 & 25.2767 & 25.5458 & 0.4046 & 0.4286 & 2.0872 \\
\hline & 22 & 26.6849 & 25.6531 & 26.1656 & 26.1623 & 26.1263 & 0.3962 & 0.1233 & 2.3981 \\
\hline & 23 & 25.0510 & 23.3744 & 24.6021 & 24.6514 & 24.7210 & 0.2579 & 0.0651 & 1.8849 \\
\hline & 24 & 27.2220 & 25.0605 & 26.6241 & 26.5886 & 26.4800 & 0.6041 & -0.0135 & 2.2091 \\
\hline & 25 & 26.2791 & 25.0645 & 25.6254 & 25.5946 & 25.4050 & 0.2529 & 0.2614 & 2.3224 \\
\hline & 26 & 29.4897 & 27.8875 & 28.4897 & 28.2351 & 28.7037 & 0.2613 & 0.2777 & 2.1777 \\
\hline & 27 & 28.1395 & 26.7127 & 27.4735 & 27.4925 & 27.8841 & 0.3197 & 0.1078 & 1.9386 \\
\hline & 28 & 33.6648 & 32.4435 & 32.8360 & 32.7968 & 32.5085 & 0.3377 & -0.2523 & 2.2225 \\
\hline \multirow{8}{*}{$\begin{array}{l}\text { 이 } \\
\text { 禾 } \\
\text { 넌 }\end{array}$} & 1 & 27.9548 & 26.5154 & 27.2260 & 27.1995 & 27.0858 & 0.3569 & 0.1274 & 2.0021 \\
\hline & 2 & 28.8129 & 27.3523 & 28.1262 & 28.1226 & 27.9042 & 0.3491 & -0.0138 & 2.1087 \\
\hline & 3 & 27.5616 & 26.1888 & 26.8446 & 26.8432 & 26.6077 & 0.2789 & 0.0939 & 2.4005 \\
\hline & 4 & 29.6000 & 28.5288 & 29.1702 & 29.1772 & 29.1327 & 0.2202 & -0.3065 & 2.3702 \\
\hline & 5 & 32.1622 & 30.6685 & 31.1935 & 31.1442 & 30.7931 & 0.3027 & 0.5650 & 2.6446 \\
\hline & 6 & 34.4487 & 32.5815 & 33.3944 & 33.4007 & 33.6511 & 0.4172 & 0.0447 & 1.9892 \\
\hline & 7 & 32.5310 & 31.3926 & 31.8374 & 31.7600 & 31.5965 & 0.2727 & 0.7486 & 2.4340 \\
\hline & 8 & 31.6390 & 30.2856 & 30.7270 & 30.6750 & 30.5062 & 0.2576 & 0.8266 & 3.2548 \\
\hline
\end{tabular}




\begin{tabular}{|c|c|c|c|c|c|c|c|c|}
\hline 10 & 354435 & 320100 & 345267 & 344511 & 344514 & 03063 & 06538 & 27593 \\
\hline 12 & 280504 & 266077 & 270681 & 270213 & 269417 & 02551 & 08292 & 36955 \\
\hline 13 & 246359 & 232334 & 239262 & 239291 & 237341 & 03178 & 00527 & 21870 \\
\hline 15 & 330924 & 323649 & 326331 & 326175 & 325021 & 01386 & 03769 & 25512 \\
\hline 16 & 296376 & 278022 & 285544 & 285221 & 282227 & 03862 & 04670 & 26062 \\
\hline 17 & 315445 & 301088 & 305492 & 304988 & 303739 & 02751 & 09564 & 36453 \\
\hline 18 & 331066 & 317052 & 321949 & 321658 & 319955 & 02696 & 05151 & 27192 \\
\hline 22 & 259366 & 239585 & 219617 & 228371 & 226170 & 04602 & 00874 & 20442 \\
\hline 23 & 319233 & 280772 & 291155 & 297013 & 299298 & 03681 & 03023 & 19294 \\
\hline 24 & 321225 & 304681 & 303281 & 309514 & 300742 & 04136 & -02103 & 20365 \\
\hline 25 & 244335 & 197839 & 235783 & 236514 & 233685 & 04031 & 02008 & 19793 \\
\hline 26 & 327918 & 306034 & 301348 & 300962 & 305571 & 02770 & 02027 & 20309 \\
\hline 27 & 268177 & 254963 & 251895 & 252010 & 251129 & 03149 & -00485 & 21638 \\
\hline 28 & 290955 & 275378 & 291020 & 290945 & 291895 & 03190 & 05211 & 26789 \\
\hline
\end{tabular}




\section{4. $\quad 3^{\text {rd }}$ MCP Raw Data Measurements}

Table 10-4: Raw Data Measurements Extracted from the Patient Group's $3^{\text {rd }}$ MCP Joint

\begin{tabular}{|c|c|c|c|c|c|c|c|c|c|}
\hline \multicolumn{10}{|c|}{ Patient Group's 3rd MCP Result } \\
\hline & & $\operatorname{Max}\left({ }^{\circ} \mathrm{C}\right)$ & $\operatorname{Min}\left({ }^{\circ} \mathrm{C}\right)$ & Mean $\left({ }^{\circ} \mathrm{C}\right)$ & Median ( $\left.{ }^{\circ} \mathrm{C}\right)$ & Mode $\left({ }^{\circ} \mathrm{C}\right)$ & STD & Skewness & Kurtosis \\
\hline \multirow{26}{*}{ 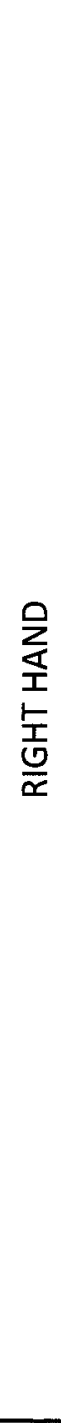 } & 1 & 33.7148 & 32.7104 & 33.0718 & 33.0629 & 32.9479 & 0.1634 & 0.6039 & 3.7875 \\
\hline & 2 & 34.2114 & 33.1590 & 33.6750 & 33.6971 & 33.9117 & 0.2670 & -0.0658 & 1.7664 \\
\hline & 3 & 33.4965 & 32.6184 & 33.1865 & 33.1878 & 33.1662 & 0.1218 & -0.5207 & 4.0262 \\
\hline & 4 & 30.0953 & 28.8356 & 29.2370 & 29.1853 & 29.1036 & 0.2460 & 1.5795 & 5.5391 \\
\hline & 5 & 29.9465 & 29.0944 & 29.5748 & 29.6065 & 29.6953 & 0.1492 & -0.4995 & 2.6517 \\
\hline & 6 & 30.5062 & 29.5176 & 29.8991 & 29.9022 & 29.9392 & 0.2060 & 0.4202 & 2.8930 \\
\hline & 7 & 33.7362 & 32.7968 & 33.3208 & 33.3427 & 33.5504 & 0.2495 & -0.2523 & 1.7555 \\
\hline & 8 & 32.7681 & 31.7849 & 32.2695 & 32.2558 & 32.1835 & 0.1719 & 0.0768 & 2.7501 \\
\hline & 9 & 30.8371 & 29.6671 & 30.0733 & 30.0216 & 29.9257 & 0.2342 & 0.7954 & 3.1084 \\
\hline & 10 & 33.4865 & 31.8650 & 32.2666 & 32.1839 & 32.1332 & 0.2519 & 1.7503 & 6.5791 \\
\hline & 11 & 33.3069 & 31.8498 & 32.4927 & 32.4796 & 32.2917 & 0.3363 & 0.2096 & 2.1556 \\
\hline & 12 & 32.6643 & 31.6147 & 32.0417 & 32.0502 & 32.1298 & 0.2147 & 0.1873 & 2.3189 \\
\hline & 13 & 28.5438 & 27.9368 & 28.2591 & 28.2519 & 28.1320 & 0.1193 & 0.2457 & 2.4683 \\
\hline & 14 & 33.7569 & 31.2424 & 32.0055 & 32.0635 & 32.2086 & 0.3585 & -0.1182 & 2.0425 \\
\hline & 15 & 32.0316 & 30.6287 & 31.4104 & 31.4638 & 31.7480 & 0.3635 & -0.3502 & 2.0057 \\
\hline & 16 & 31.7239 & 27.4682 & 30.1075 & 30.1323 & 31.0596 & 0.3250 & -0.1642 & 1.9944 \\
\hline & 17 & 34.5043 & 32.6828 & 33.5386 & 33.5036 & 33.0606 & 0.4867 & 0.0936 & 1.8333 \\
\hline & 18 & 35.8645 & 33.8081 & 34.8787 & 34.8837 & 34.6942 & 0.5340 & -0.0691 & 2.0292 \\
\hline & 19 & 25.1731 & 23.4249 & 24.1959 & 24.1743 & 24.3763 & 0.4166 & 0.1469 & 1.9079 \\
\hline & 20 & 29.8062 & 27.8228 & 28.8122 & 28.8338 & 27.8980 & 0.5143 & -0.0740 & 2.0605 \\
\hline & 21 & 30.0327 & 28.6609 & 29.1920 & 29.1315 & 29.8830 & 0.3151 & 0.6092 & 2.7787 \\
\hline & 22 & 28.7293 & 25.0533 & 27.8379 & 27.8983 & 27.9136 & 0.3936 & -0.0867 & 2.0032 \\
\hline & 23 & 30.2791 & 28.4391 & 29.3808 & 29.3629 & 29.8145 & 0.4283 & 0.0851 & 2.5326 \\
\hline & 24 & 25.3656 & 23.9095 & 24.6842 & 24.6842 & 24.2054 & 0.3701 & -0.1177 & 1.8501 \\
\hline & 25 & 33.6240 & 31.9635 & 32.9044 & 32.9044 & 32.9795 & 0.4684 & 0.0996 & 2.3867 \\
\hline & 26 & 34.5301 & 32.9509 & 33.7361 & 33.7361 & 33.7204 & 0.4267 & 0.0645 & 1.8996 \\
\hline \multirow{8}{*}{$\begin{array}{l}\text { 至 } \\
\text { 志 } \\
\frac{1}{\amalg}\end{array}$} & 1 & 33.3284 & 32.5229 & 32.9428 & 32.9407 & 32.9695 & 0.1485 & -0.0659 & 2.8655 \\
\hline & 2 & 33.3387 & 32.5822 & 33.0151 & 33.0223 & 32.9863 & 0.1379 & -0.6092 & 3.3563 \\
\hline & 3 & 34.0902 & 32.8566 & 33.6180 & 33.6542 & 33.7043 & 0.2345 & -0.8674 & 3.7121 \\
\hline & 4 & 30.7639 & 29.3486 & 29.7821 & 29.6967 & 29.6079 & 0.3071 & 0.9006 & 3.0437 \\
\hline & 5 & 29.3915 & 28.6921 & 29.0793 & 29.0833 & 29.0721 & 0.1437 & -0.2263 & 2.5309 \\
\hline & 6 & 29.2876 & 27.8077 & 28.4814 & 28.4155 & 28.1533 & 0.3390 & 0.4014 & 2.1294 \\
\hline & 7 & 33.6648 & 32.5373 & 33.1810 & 33.2208 & 33.5074 & 0.2846 & -0.4192 & 2.1694 \\
\hline & 8 & 33.0054 & 31.5159 & 32.2548 & 32.2342 & 31.8647 & 0.3351 & 0.2685 & 2.1979 \\
\hline
\end{tabular}




\begin{tabular}{|c|c|c|c|c|c|c|c|c|}
\hline 9 & 31.7994 & 29.4376 & 30.1800 & 30.1137 & 30.1174 & 0.4380 & 0.8169 & 3.5398 \\
\hline 10 & 33.4435 & 32.0100 & 32.5267 & 32.4658 & 32.4514 & 0.3063 & 0.6538 & 2.7593 \\
\hline 11 & 33.4430 & 32.1325 & 32.7466 & 32.6960 & 32.9335 & 0.3346 & 0.2709 & 1.9305 \\
\hline 12 & 32.7076 & 31.4691 & 31.9673 & 31.9560 & 31.9125 & 0.2450 & 0.2584 & 2.4530 \\
\hline 13 & 29.4667 & 28.5811 & 28.9966 & 28.9690 & 28.8349 & 0.1869 & 0.3749 & 2.3855 \\
\hline 14 & 34.7949 & 32.1885 & 33.8769 & 33.8454 & 33.8952 & 0.3856 & 0.3129 & 2.1624 \\
\hline 15 & 31.3469 & 29.7801 & 30.6283 & 30.6214 & 30.6214 & 0.3862 & -0.0570 & 2.1561 \\
\hline 16 & 29.1710 & 27.1973 & 28.6770 & 27.6564 & 27.3389 & 0.7253 & 0.0752 & 2.0548 \\
\hline 17 & 33.9058 & 29.5645 & 32.2060 & 32.1627 & 32.0056 & 0.2450 & 0.2818 & 2.8529 \\
\hline 18 & 35.7649 & 33.3544 & 34.4607 & 34.5160 & 33.6049 & 0.6145 & -0.0351 & 1.9241 \\
\hline 19 & 35.0343 & 33.6675 & 34.3356 & 34.3142 & 34.3142 & 0.3707 & 0.2025 & 1.8702 \\
\hline 20 & 29.3396 & 27.8453 & 28.7281 & 28.7629 & 28.9530 & 0.3426 & -0.3860 & 2.4419 \\
\hline 21 & 34.9442 & 31.5677 & 33.1223 & 33.1265 & 33.5198 & 0.4386 & -0.1122 & 2.0793 \\
\hline 22 & 32.5697 & 30.5818 & 31.6682 & 31.6954 & 31.5995 & 0.4936 & -0.2367 & 2.0971 \\
\hline 23 & 28.9913 & 26.1337 & 27.7096 & 27.7864 & 27.8466 & 0.7246 & -0.1876 & 1.9907 \\
\hline 24 & 26.2162 & 24.2209 & 25.1224 & 25.0883 & 24.0883 & 0.4907 & 0.1735 & 1.9479 \\
\hline 25 & 26.8164 & 24.9949 & 25.9622 & 25.9477 & 25.9324 & 0.4664 & -0.0413 & 1.8409 \\
\hline 26 & 34.0127 & 31.0826 & 32.4938 & 32.4595 & 32.2848 & 0.1999 & 0.4287 & 2.7276 \\
\hline GE & 31.7956 & 30.0996 & 30.9580 & 30.9317 & 30.8998 & 0.3357 & 0.1313 & 2.5451 \\
\hline
\end{tabular}




\subsection{Ankle Raw Data Measurement}

Table 10-5: Raw Data Measurements Extracted from the Control Group's Ankle Joint

\begin{tabular}{|c|c|c|c|c|c|c|c|c|c|}
\hline \multicolumn{10}{|c|}{ Control Group's Ankle Result } \\
\hline & & $\operatorname{Max}\left({ }^{\circ} \mathrm{C}\right)$ & $\operatorname{Min}\left({ }^{\circ} \mathrm{C}\right)$ & Mean $\left({ }^{\circ} \mathrm{C}\right)$ & Median ( $\left.{ }^{\circ} \mathrm{C}\right)$ & Mode $\left({ }^{\circ} \mathrm{C}\right)$ & STD & Skewness & Kurtosis \\
\hline \multirow{28}{*}{ 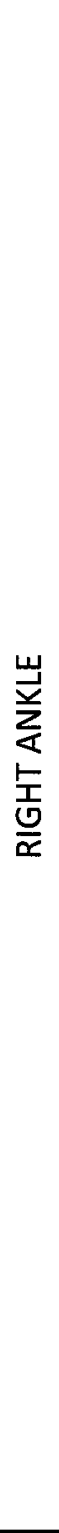 } & 1 & 27.8534 & 24.3390 & 26.3450 & 26.4004 & 25.8871 & 0.7840 & -0.4238 & 2.7291 \\
\hline & 2 & 28.0622 & 24.9064 & 26.8268 & 26.8945 & 26.6560 & 0.6144 & -0.4893 & 3.0242 \\
\hline & 3 & 30.3196 & 25.4118 & 28.0196 & 28.0813 & 27.9681 & 0.9236 & -0.5847 & 4.0474 \\
\hline & 4 & 31.3140 & 27.5931 & 29.5924 & 29.6363 & 29.7629 & 0.7134 & -0.3664 & 3.3930 \\
\hline & 5 & 31.0081 & 28.2520 & 30.0832 & 30.1549 & 30.5202 & 0.5240 & -1.1285 & 5.0854 \\
\hline & 6 & 31.6303 & 27.5328 & 29.4204 & 29.4151 & 29.4411 & 0.7149 & 0.0599 & 2.7210 \\
\hline & 7 & 31.1920 & 28.2710 & 29.4566 & 29.4217 & 29.6504 & 0.6205 & 0.5463 & 3.2773 \\
\hline & 8 & 31.7405 & 29.5617 & 30.6521 & 30.7432 & 30.8921 & 0.4221 & -0.3795 & 2.5546 \\
\hline & 9 & $x$ & $x$ & $x$ & $x$ & $x$ & $x$ & $x$ & $x$ \\
\hline & 10 & 34.9784 & 31.7331 & 33.3145 & 33.2851 & 32.4729 & 0.6849 & 0.1068 & 2.8023 \\
\hline & 11 & 29.1283 & 26.6257 & 27.8146 & 27.8080 & 27.5780 & 0.5252 & 0.0249 & 2.4388 \\
\hline & 12 & 30.2399 & 27.1838 & 28.6064 & 28.6454 & 28.9014 & 0.6628 & -0.0116 & 2.5140 \\
\hline & 13 & 27.8756 & 24.0867 & 26.0094 & 26.0677 & 26.2817 & 0.7391 & -0.2199 & 2.6871 \\
\hline & 14 & 30.1687 & 26.0867 & 28.3108 & 28.2940 & 28.0427 & 0.6616 & 0.1693 & 2.7739 \\
\hline & 15 & 29.2964 & 26.3658 & 28.0168 & 28.0482 & 28.2502 & 0.5644 & -0.4897 & 3.1700 \\
\hline & 16 & 31.0008 & 25.7718 & 28.4693 & 28.5745 & 28.3161 & 1.0322 & -0.4058 & 3.2326 \\
\hline & 17 & 30.8264 & 27.8347 & 29.1061 & 28.9715 & 28.7252 & 0.6622 & 0.5427 & 2.6081 \\
\hline & 18 & 32.1255 & 29.4612 & 30.9153 & 30.9352 & 30.8489 & 0.5341 & -0.1654 & 2.5441 \\
\hline & 19 & 29.8263 & 26.5570 & 29.4627 & 29.3073 & 28.7905 & 0.6588 & -0.2462 & 1.9420 \\
\hline & 20 & 32.5896 & 30.4446 & 31.3651 & 31.0491 & 31.1922 & 0.5797 & -0.2351 & 2.7758 \\
\hline & 21 & 30.9292 & 27.8029 & 29.6974 & 29.3199 & 29.3742 & 0.7554 & -0.0365 & 2.6387 \\
\hline & 22 & 32.9281 & 29.8512 & 31.1047 & 30.8525 & 30.7103 & 0.7215 & -0.5239 & 2.9612 \\
\hline & 23 & 30.1367 & 26.8077 & 28.8772 & 29.3320 & 29.1770 & 1.0009 & -0.3596 & 2.5130 \\
\hline & 24 & 31.9716 & 29.1119 & 30.3722 & 30.1045 & 29.9378 & 0.8599 & 0.5218 & 2.2432 \\
\hline & 25 & 29.6305 & 26.9471 & 29.2348 & 28.9152 & 28.8713 & 1.0123 & 0.2214 & 2.4685 \\
\hline & 26 & 30.5911 & 27.9682 & 29.3392 & 29.8277 & 29.4603 & 0.9171 & -0.5579 & 2.9956 \\
\hline & 27 & 29.6110 & 26.6012 & 28.6615 & 29.1930 & 28.5927 & 0.9748 & -0.2506 & 2.7401 \\
\hline & 28 & 32.4434 & 28.6924 & 30.6604 & 30.6727 & 30.4473 & 0.9166 & 0.1906 & 2.8489 \\
\hline \multirow{6}{*}{ 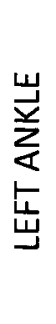 } & 1 & 27.7992 & 24.2147 & 26.3368 & 26.4423 & 26.2974 & 0.7979 & -0.5758 & 2.6493 \\
\hline & 2 & 28.2560 & 25.0064 & 26.9612 & 27.0592 & 27.5452 & 0.7023 & -0.3186 & 2.4534 \\
\hline & 3 & 30.4237 & 25.3852 & 28.1460 & 28.2673 & 28.5417 & 0.9699 & -0.5259 & 3.9542 \\
\hline & 4 & 31.4456 & 27.4331 & 29.8942 & 29.9900 & 30.3721 & 0.8396 & -0.6696 & 3.3835 \\
\hline & 5 & 31.1236 & 29.0419 & 30.3540 & 30.4172 & 30.3345 & 0.4163 & -0.8660 & 3.9681 \\
\hline & 6 & 31.8208 & 27.0867 & 29.8033 & 29.7660 & 29.6988 & 0.7353 & -0.0782 & 3.2760 \\
\hline
\end{tabular}




\begin{tabular}{|c|c|c|c|c|c|c|c|c|}
\hline 7 & 30.8784 & 27.9840 & 28.9066 & 28.0284 & 28.6169 & 0.6264 & 0.6696 & 3.1223 \\
\hline 8 & 32.9931 & 29.8229 & 31.4291 & 31.4265 & 31.4022 & 0.5598 & -0.1961 & 4.1395 \\
\hline 9 & $x$ & $x$ & $x$ & $x$ & $x$ & $x$ & $x$ & $x$ \\
\hline 10 & 34.5106 & 31.4597 & 33.2062 & 33.2128 & 33.1790 & 0.6601 & -0.2811 & 2.4572 \\
\hline 11 & 29.1097 & 25.4132 & 27.3286 & 27.4040 & 28.1753 & 0.7374 & -0.2724 & 2.5908 \\
\hline 12 & 30.0197 & 26.6720 & 28.3907 & 28.4058 & 28.2445 & 0.6768 & 0.0678 & 2.1802 \\
\hline 13 & 27.7304 & 23.8300 & 25.7197 & 25.7671 & 25.6802 & 0.7701 & -0.0661 & 2.7462 \\
\hline 14 & 30.1914 & 26.6159 & 28.4660 & 28.4846 & 28.2729 & 0.6915 & -0.0404 & 2.5012 \\
\hline 15 & 29.0820 & 25.5391 & 27.5189 & 27.5407 & 27.7655 & 0.6283 & -0.2704 & 3.1045 \\
\hline 16 & 31.2238 & 25.7582 & 28.5277 & 28.5520 & 29.0414 & 1.1101 & -0.2175 & 3.2410 \\
\hline 17 & 31.2183 & 26.8602 & 28.6010 & 28.5094 & 28.3556 & 0.8842 & 0.5803 & 3.3786 \\
\hline 18 & 31.6397 & 28.5397 & 30.2898 & 30.3592 & 30.1278 & 0.6608 & -0.1536 & 2.2988 \\
\hline 19 & 29.7766 & 27.6643 & 28.9703 & 28.8762 & 29.1723 & 0.7708 & -0.1324 & 2.8480 \\
\hline 20 & 32.6514 & 29.8683 & 31.5831 & 31.5936 & 31.7490 & 0.5322 & 0.0033 & 2.1769 \\
\hline 21 & 30.7527 & 27.3452 & 29.3974 & 29.7267 & 29.6995 & 0.6709 & -0.3191 & 3.1951 \\
\hline 22 & 32.0124 & 29.6604 & 30.9160 & 31.0030 & 30.8464 & 0.8273 & -0.5124 & 2.9449 \\
\hline 23 & 30.3793 & 27.1248 & 29.2071 & 29.2403 & 28.8805 & 0.9702 & -0.2171 & 2.5098 \\
\hline 24 & 31.3834 & 29.7905 & 30.6065 & 30.0603 & 29.9678 & 0.8541 & 0.4379 & 1.7530 \\
\hline 25 & 30.6415 & 26.8090 & 28.8688 & 28.7459 & 28.0516 & 0.9862 & 0.2520 & 2.3754 \\
\hline 26 & 29.7657 & 26.6074 & 29.1131 & 29.2025 & 28.9445 & 0.8645 & -0.5504 & 3.1270 \\
\hline 27 & 29.9687 & 25.9119 & 28.2151 & 27.9956 & 28.4774 & 0.9935 & 0.0630 & 2.4197 \\
\hline 28 & 30.7482 & 28.9589 & 30.1347 & 30.5131 & 30.3883 & 0.6979 & -0.5525 & 2.8887 \\
\hline GE & 30.5284 & 27.2118 & 29.0405 & 29.0393 & 29.0178 & 0.7513 & -0.1742 & 2.8876 \\
\hline
\end{tabular}


10.6. Ankle Raw Data Measurement

Table 10-6: Raw Data Measurements Extracted from the Patient Group's Ankle Joint

\begin{tabular}{|c|c|c|c|c|c|c|c|c|c|}
\hline \multicolumn{10}{|c|}{ Patient Group's Ankle Result } \\
\hline & & $\operatorname{Max}\left({ }^{\circ} \mathrm{C}\right)$ & $\operatorname{Min}\left({ }^{\circ} \mathrm{C}\right)$ & Mean $\left({ }^{\circ} \mathrm{C}\right)$ & Median $\left({ }^{\circ} \mathrm{C}\right)$ & Mode $\left({ }^{\circ} \mathrm{C}\right)$ & STD & Skewness & Kurtosis \\
\hline \multirow{25}{*}{ 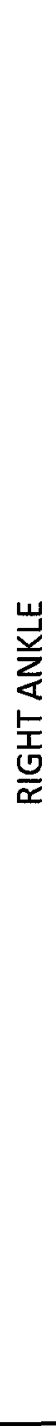 } & 1 & 31.9697 & 27.4458 & 30.1467 & 30.2664 & 30.7483 & 0.8949 & -0.8846 & 3.4977 \\
\hline & 2 & 31.8378 & 28.9974 & 30.5990 & 30.5732 & 30.5602 & 0.5256 & -0.2762 & 3.0984 \\
\hline & 3 & 31.5938 & 28.0131 & 29.8346 & 29.7937 & 29.2691 & 0.6992 & 0.0547 & 2.7760 \\
\hline & 4 & 28.3446 & 23.7718 & 26.1864 & 26.1619 & 26.0533 & 0.9359 & -0.3165 & 3.3294 \\
\hline & 5 & 30.5566 & 26.2503 & 28.5739 & 28.5958 & 29.1154 & 0.9412 & -0.1192 & 2.1611 \\
\hline & 6 & 32.3034 & 29.5587 & 30.9645 & 30.9590 & 30.7794 & 0.5630 & -0.0344 & 2.5859 \\
\hline & 7 & 32.3837 & 29.9208 & 31.2544 & 31.2406 & 31.2797 & 0.4680 & -0.2115 & 2.8418 \\
\hline & 8 & 28.6185 & 25.1772 & 27.1556 & 28.5541 & 26.8384 & 0.7007 & -0.4905 & 3.3060 \\
\hline & 9 & 30.8412 & 27.8498 & 29.3508 & 29.3229 & 29.2597 & 0.5475 & 0.2320 & 3.1634 \\
\hline & 10 & 32.0274 & 28.5977 & 30.5722 & 30.5933 & 30.3161 & 0.6921 & -0.2232 & 2.4320 \\
\hline & 11 & 30.8031 & 27.0346 & 28.9079 & 28.8938 & 28.9548 & 0.8914 & 0.2390 & 2.6156 \\
\hline & 12 & 29.8828 & 26.8903 & 28.5981 & 28.6707 & 28.9467 & 0.5749 & -0.3871 & 2.6858 \\
\hline & 13 & 31.8759 & 27.3671 & 28.9793 & 29.2304 & 29.2217 & 0.8450 & 0.4335 & 3.5910 \\
\hline & 14 & 34.2322 & 29.1906 & 31.7191 & 31.8295 & 31.9985 & 0.9902 & -0.1936 & 2.8500 \\
\hline & 15 & 34.2493 & 27.4005 & 31.0979 & 31.2287 & 30.0267 & 1.5685 & -0.3747 & 3.0381 \\
\hline & 16 & 33.9487 & 30.1974 & 31.9086 & 31.8290 & 31.2503 & 0.7747 & 0.2423 & 2.5045 \\
\hline & 17 & 32.9091 & 28.9800 & 31.2136 & 31.2237 & 30.9407 & 0.8333 & -0.4065 & 2.9554 \\
\hline & 18 & 34.3314 & 31.8213 & 33.2495 & 33.3554 & 33.6241 & 0.6395 & -0.4734 & 2.4174 \\
\hline & 20 & 32.6834 & 27.1237 & 30.2634 & 30.3650 & 29.4188 & 1.2035 & -0.8318 & 4.1599 \\
\hline & 21 & 32.9021 & 28.8227 & 31.1374 & 31.2526 & 31.5830 & 0.8427 & -0.4172 & 2.6412 \\
\hline & 22 & 34.4999 & 31.8573 & 33.2832 & 33.2797 & 32.7242 & 0.6186 & -0.1386 & 2.2624 \\
\hline & 23 & 34.2893 & 31.2636 & 32.8094 & 32.8440 & 32.4451 & 0.6762 & -0.2113 & 2.5723 \\
\hline & 24 & 32.2422 & 28.2828 & 30.4856 & 30.6119 & 30.9718 & 0.8704 & -0.2025 & 2.6323 \\
\hline & 25 & 35.7159 & 31.6773 & 34.1794 & 34.6624 & 34.4871 & 0.9190 & -0.3687 & 2.6403 \\
\hline & 26 & 33.1502 & 28.4887 & 31.4680 & 31.6624 & 32.3195 & 1.0709 & -0.7667 & 3.3049 \\
\hline \multirow{8}{*}{ 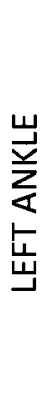 } & 1 & 31.7781 & 28.1375 & 30.1593 & 30.1379 & 30.1420 & 0.6959 & -0.2428 & 2.7192 \\
\hline & 2 & 32.0099 & 29.3693 & 30.6630 & 30.6490 & 30.3388 & 0.5172 & 0.1689 & 2.6764 \\
\hline & 3 & 32.2655 & 29.0651 & 30.6455 & 30.6757 & 30.9232 & 0.6617 & -0.3464 & 3.3788 \\
\hline & 4 & 27.2027 & 23.3602 & 25.3303 & 25.2867 & 24.5376 & 0.7872 & -0.1853 & 3.2700 \\
\hline & 5 & 29.8181 & 26.3079 & 28.0958 & 28.0949 & 28.2348 & 0.6956 & 0.0993 & 2.6651 \\
\hline & 6 & 32.6961 & 29.4706 & 31.0224 & 31.0170 & 31.0411 & 0.6052 & 0.1691 & 3.1176 \\
\hline & 7 & 33.0406 & 30.8673 & 32.0951 & 32.1426 & 32.2373 & 0.3800 & -0.3399 & 3.4557 \\
\hline & 8 & 28.6609 & 25.5501 & 27.2704 & 27.2709 & 26.9252 & 0.6455 & -0.2053 & 2.7689 \\
\hline
\end{tabular}




\begin{tabular}{|c|c|c|c|c|c|c|c|c|}
\hline 9 & 29.6399 & 27.0290 & 28.2815 & 28.2502 & 28.2404 & 0.5539 & -0.0599 & 2.4471 \\
\hline 10 & 31.7973 & 28.5965 & 30.3848 & 30.3745 & 30.0864 & 0.6547 & -0.2210 & 2.7432 \\
\hline 11 & 30.7617 & 27.5518 & 29.1195 & 29.1461 & 28.9778 & 0.6823 & 0.1117 & 2.6385 \\
\hline 12. & 29.3956 & 26.2974 & 27.9989 & 28.0587 & 27.9412 & 0.6883 & -0.2582 & 2.3007 \\
\hline 13 & 31.3847 & 26.7882 & 28.9555 & 29.0136 & 29.2294 & 0.9193 & -0.0121 & 2.5727 \\
\hline 14 & 34.2527 & 29.4882 & 31.9462 & 31.9396 & 32.0430 & 0.8612 & -0.2666 & 2.9813 \\
\hline 15 & 34.3253 & 28.2931 & 31.7337 & 31.8016 & 31.8168 & 1.3188 & -0.3669 & 3.0688 \\
\hline 16 & 33.7179 & 29.2543 & 32.1207 & 32.0708 & 31.8838 & 0.8039 & -0.6706 & 5.5077 \\
\hline 17 & 33.4469 & 29.7682 & 31.7048 & 31.7038 & 31.7122 & 0.7278 & -0.0710 & 3.0021 \\
\hline 18 & 34.5846 & 31.6118 & 33.4810 & 33.6042 & 34.0462 & 0.6452 & -0.9163 & 3.8534 \\
\hline 19 & 33.5753 & 28.2957 & 31.9050 & 32.2066 & 32.0904 & 1.2235 & -1.0620 & 3.7026 \\
\hline 20 & 32.7005 & 26.5773 & 30.5685 & 30.8051 & 30.8116 & 1.3647 & -1.0661 & 4.0180 \\
\hline 21 & 33.0291 & 29.9618 & 31.1890 & 31.2831 & 31.1346 & 0.7933 & -0.1496 & 3.5631 \\
\hline 22 & 35.0536 & 32.7623 & 33.7898 & 33.7332 & 33.3564 & 0.5010 & 0.5061 & 2.7365 \\
\hline 23 & 34.0093 & 30.2121 & 32.4665 & 32.5318 & 32.5521 & 0.7287 & -0.4790 & 2.9620 \\
\hline 24 & 31.2956 & 27.6175 & 29.6139 & 29.7461 & 29.3862 & 0.8322 & -0.2507 & 2.6001 \\
\hline 25 & 35.6496 & 31.9042 & 34.0645 & 34.1377 & 34.4235 & 0.7432 & -0.3424 & 2.7684 \\
\hline 26 & 33.4440 & 29.4119 & 32.0393 & 32.2627 & 32.4511 & 0.8719 & -0.6533 & 3.2089 \\
\hline $\mathrm{GE}$ & 32.3336 & 28.5495 & 30.6305 & 30.7185 & 30.6302 & 0.7922 & -0.2760 & 3.0187 \\
\hline
\end{tabular}


10.7. Knee Raw Data Measurement

Table 10-7: Raw Data Measurements Extracted from the Control Group's Knee Joint

\begin{tabular}{|c|c|c|c|c|c|c|c|c|c|}
\hline \multicolumn{10}{|c|}{ Control Group's Knee Result } \\
\hline & & $\operatorname{Max}\left({ }^{\circ} \mathrm{C}\right)$ & $\operatorname{Min}\left({ }^{\circ} \mathrm{C}\right)$ & Mean $\left({ }^{\circ} \mathrm{C}\right)$ & Median ( ${ }^{\circ} \mathrm{C}$ ) & Mode $\left({ }^{\circ} \mathrm{C}\right)$ & STD & Skewness & Kurtosis \\
\hline \multirow{23}{*}{ 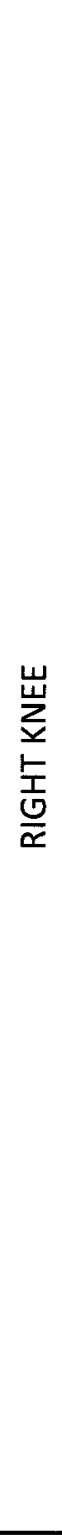 } & 1 & 30.2493 & 27.0934 & 28.7182 & 28.8543 & 29.0781 & 0.5696 & -0.3387 & 2.7822 \\
\hline & 2 & 29.8330 & 27.8514 & 29.1100 & 29.1639 & 29.3204 & 0.3243 & -1.0887 & 4.4812 \\
\hline & 3 & 30.3823 & 27.6369 & 29.6064 & 29.6745 & 29.7189 & 0.4351 & -1.5978 & 6.4680 \\
\hline & 4 & 33.0060 & 28.4765 & 31.0202 & 31.1515 & 31.4141 & 0.8399 & -0.4804 & 2.9319 \\
\hline & 5 & 31.3194 & 28.8498 & 30.3849 & 30.4923 & 30.6025 & 0.4187 & -0.9371 & 3.9023 \\
\hline & 6 & 31.9233 & 28.7535 & 30.9183 & 31.0716 & 31.3124 & 0.5506 & -1.0887 & 3.7233 \\
\hline & 7 & 31.1884 & 28.4174 & 29.9771 & 29.9628 & 29.9037 & 0.5265 & -0.3303 & 2.8037 \\
\hline & 8 & 32.3423 & 30.2193 & 31.3156 & 31.3332 & 31.3769 & 0.3390 & -0.2411 & 2.8205 \\
\hline & 9 & $x$ & $x$ & $x$ & $x$ & $x$ & $x$ & $x$ & $x$ \\
\hline & 10 & 34.9168 & 31.7518 & 32.5376 & 32.4879 & 32.4435 & 0.2537 & -0.2411 & 2.8205 \\
\hline & 11 & 30.6826 & 26.7517 & 29.4577 & 29.6591 & 29.7035 & 0.7381 & -1.1407 & 3.7774 \\
\hline & 12 & 30.9250 & 26.6305 & 29.0497 & 29.1184 & 28.7909 & 0.9487 & -0.2888 & 2.1461 \\
\hline & 13 & 28.7083 & 25.6913 & 27.7761 & 27.9298 & 28.2299 & 0.6067 & -1.0905 & 3.6389 \\
\hline & 14 & 30.6606 & 26.1121 & 29.2306 & 29.3109 & 29.4147 & 0.5991 & -0.7396 & 3.6096 \\
\hline & 15 & 29.6450 & 28.4024 & 29.1597 & 29.1556 & 29.1110 & 0.1573 & -0.2628 & 3.6784 \\
\hline & 16 & 32.1769 & 28.3350 & 30.6243 & 30.7056 & 31.4509 & 0.9255 & -0.3442 & 2.1790 \\
\hline & 17 & 30.9753 & 28.2957 & 29.7534 & 29.8431 & 30.0277 & 0.5805 & -0.2536 & 2.1453 \\
\hline & 18 & 31.7626 & 28.6323 & 29.9560 & 30.0130 & 30.0425 & 0.5676 & -0.0333 & 2.5365 \\
\hline & 24 & 32.2327 & 29.8022 & 30.9163 & 30.9013 & 31.0103 & 0.4961 & 0.1217 & 2.3382 \\
\hline & 25 & 32.7248 & 30.2190 & 31.5015 & 31.5960 & 31.8871 & 0.5328 & -0.2720 & 2.1915 \\
\hline & 26 & 30.9320 & 28.4579 & 29.6620 & 29.6253 & 29.8871 & 0.4949 & 0.2134 & 2.8147 \\
\hline & 27 & 32.9695 & 29.5539 & 31.1985 & 30.4198 & 30.4198 & 0.7611 & 0.1838 & 2.5940 \\
\hline & 28 & 32.0975 & 29.6307 & 30.6450 & 30.6584 & 303.6584 & 0.3394 & 0.2175 & 2.1083 \\
\hline \multirow{6}{*}{ 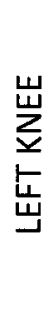 } & 1 & 31.9546 & 28.1278 & 30.1671 & 30.0943 & 29.8948 & 0.5649 & 0.2520 & 2.9179 \\
\hline & 2 & 31.2692 & 28.4383 & 29.6503 & 29.6549 & 29.6921 & 0.3858 & -0.0075 & 3.5097 \\
\hline & 3 & 30.5880 & 27.3428 & 29.5864 & 29.6375 & 29.6523 & 0.4350 & -1.4072 & 6.3791 \\
\hline & 4 & 32.6894 & 29.0360 & 31.0510 & 31.2172 & 31.5961 & 0.7624 & -0.4195 & 2.4765 \\
\hline & 5 & 31.2099 & 27.9969 & 29.7904 & 29.8810 & 30.3086 & 0.6447 & -0.3787 & 2.5655 \\
\hline & 6 & 32.3288 & 29.2670 & 31.0810 & 31.1884 & 31.3562 & 0.5707 & -0.5929 & 2.7631 \\
\hline
\end{tabular}




\begin{tabular}{|c|c|c|c|c|c|c|c|c|}
\hline 7 & 31.9523 & 28.4248 & 30.0810 & 30.0955 & 30.6396 & 0.6653 & -0.0310 & 2.3417 \\
\hline 8 & 32.5085 & 29.9613 & 31.1837 & 31.2383 & 31.3186 & 0.4801 & -0.1617 & 2.4846 \\
\hline 9 & $x$ & $x$ & $x$ & $x$ & $x$ & $x$ & $x$ & $x$ \\
\hline 10 & $x$ & $x$ & $x$ & $x$ & $x$ & $x$ & $x$ & $x$ \\
\hline 11 & 30.1904 & 26.1274 & 28.6153 & 28.8127 & 29.4147 & 0.8386 & -0.7738 & 2.8185 \\
\hline 12 & 31.3339 & 25.9748 & 28.9712 & 29.0738 & 29.8297 & 1.0518 & -0.3539 & 2.2779 \\
\hline 13 & 29.8069 & 26.0968 & 28.4322 & 28.5889 & 28.8574 & 0.6943 & -0.8351 & 3.2502 \\
\hline 14 & 30.6679 & 26.5846 & 29.1372 & 29.2738 & 29.5185 & 0.7781 & -0.6062 & 2.7147 \\
\hline 15 & 31.3489 & 27.9526 & 29.0225 & 28.9696 & 28.8430 & 0.3935 & 0.7195 & 4.7714 \\
\hline 16 & 31.7637 & 28.8057 & 30.5102 & 30.5222 & 30.3752 & 0.6596 & -0.2578 & 2.2600 \\
\hline 17 & 31.4511 & 29.1911 & 30.4602 & 30.4841 & 30.7556 & 0.4091 & -0.3273 & 2.7163 \\
\hline 18 & 32.5668 & 30.1332 & 31.8815 & 31.9448 & 32.1332 & 0.3070 & -0.5449 & 2.6813 \\
\hline 19 & 31.0112 & 28.9556 & 30.0171 & 30.0405 & 29.9449 & 0.4350 & 0.0621 & 2.7336 \\
\hline 20 & 31.2127 & 29.3340 & 30.2880 & 30.3099 & 30.6817 & 0.4085 & 0.0364 & 2.4442 \\
\hline 21 & 32.1106 & 29.4588 & 30.8626 & 30.7905 & 30.6371 & 0.5970 & 0.0528 & 2.3730 \\
\hline 22 & 32.6867 & 31.3312 & 31.3474 & 31.2515 & 31.1200 & 0.6045 & 0.3256 & 2.0612 \\
\hline 23 & 32.1782 & 29.3177 & 30.9403 & 31.0435 & 31.1517 & 0.6302 & -0.4218 & 2.4431 \\
\hline 24 & 32.5899 & 29.5267 & 31.2324 & 31.3655 & 31.2396 & 0.7146 & -0.3546 & 2.2461 \\
\hline 25 & 33.1541 & 30.6279 & 31.9397 & 31.9428 & 31.7582 & 0.4955 & -0.2256 & 2.9612 \\
\hline 26 & 30.5725 & 29.0539 & 29.3215 & 29.3142 & 29.1580 & 0.5034 & -0.0045 & 2.6146 \\
\hline 27 & 33.1962 & 28.0539 & 30.3078 & 30.9401 & 27.8857 & 1.8433 & -0.0791 & 1.9894 \\
\hline 28 & 32.4306 & 30.4098 & 31.3831 & 31.3753 & 31.2556 & 0.4796 & 0.1419 & 2.2115 \\
\hline & 31.5713 & 28.5362 & 30.1888 & 30.2456 & 35.5173 & 0.6096 & -0.2989 & 2.9082 \\
\hline
\end{tabular}


10.8. Knee Raw Data Measurement

Table 10-8: Raw Data Measurements Extracted from the Patient Group's Knee Joint

\begin{tabular}{|c|c|c|c|c|c|c|c|c|c|}
\hline \multicolumn{10}{|c|}{ Patient Group's Knee Result } \\
\hline & & $\operatorname{Max}\left({ }^{\circ} \mathrm{C}\right)$ & $\operatorname{Min}\left({ }^{\circ} \mathrm{C}\right)$ & Mean $\left({ }^{\circ} \mathrm{C}\right)$ & Median ( $\left.{ }^{\circ} \mathrm{C}\right)$ & Mode $\left({ }^{\circ} \mathrm{C}\right)$ & STD & Skewness & Kurtosis \\
\hline \multirow{26}{*}{ 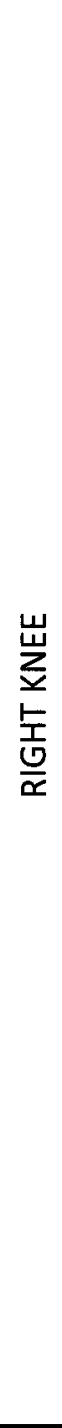 } & 1 & 31.8352 & 28.9828 & 30.6760 & 30.7776 & 31.0996 & 0.5633 & -0.6414 & 2.8053 \\
\hline & 2 & 31.6898 & 29.8763 & 30.7146 & 30.7316 & 30.8564 & 0.3305 & -0.1307 & 2.4253 \\
\hline & 3 & 31.8352 & 28.9828 & 30.6760 & 30.7776 & 31.0996 & 0.5633 & -0.6414 & 2.8053 \\
\hline & 4 & 29.0738 & 25.9060 & 28.0741 & 28.1629 & 28.2454 & 0.5395 & -1.1527 & 4.6386 \\
\hline & 5 & 30.7923 & 28.6024 & 30.0984 & 30.2120 & 30.5576 & 0.4687 & -0.7398 & 2.6766 \\
\hline & 6 & 31.2748 & 28.6398 & 30.1301 & 30.1751 & 30.5796 & 0.4721 & -0.4066 & 2.3680 \\
\hline & 7 & 32.0746 & 28.6530 & 31.3990 & 31.4206 & 31.5081 & 0.2433 & -0.1090 & 2.6753 \\
\hline & 8 & 29.8076 & 27.6595 & 28.7702 & 28.7760 & 28.8878 & 0.3868 & 0.1107 & 2.5216 \\
\hline & 9 & 29.0441 & 26.7597 & 27.9101 & 27.8551 & 27.7574 & 0.4155 & 0.4489 & 2.7057 \\
\hline & 10 & 31.9810 & 29.9032 & 31.1506 & 31.1880 & 31.4214 & 0.3226 & -0.5389 & 3.0899 \\
\hline & 11 & 30.4033 & 28.9828 & 29.9602 & 30.0130 & 30.0720 & 0.2136 & -1.2839 & 4.6348 \\
\hline & 12 & 31.2724 & 28.9586 & 30.2112 & 30.2908 & 30.4745 & 0.3652 & -0.2989 & 2.3884 \\
\hline & 13 & 30.5438 & 28.1095 & 29.3482 & 29.3480 & 29.3184 & 0.3617 & -0.1871 & 3.5468 \\
\hline & 14 & 33.6014 & 26.3101 & 31.4178 & 31.1578 & 30.9722 & 0.3467 & 0.9371 & 2.8288 \\
\hline & 15 & 34.1353 & 25.8397 & 29.5971 & 29.3221 & 29.8995 & 0.4684 & 1.4689 & 4.3860 \\
\hline & 16 & 34.1020 & 25.4773 & 29.5329 & 29.4123 & 29.6651 & 0.5238 & 0.6582 & 2.8727 \\
\hline & 17 & 36.0924 & 26.0837 & 31.8603 & 31.9895 & 32.6804 & 0.0750 & -0.2713 & 1.9801 \\
\hline & 18 & 34.6762 & 27.4921 & 31.7774 & 31.7178 & 31.8732 & 0.4497 & 0.8105 & 3.8275 \\
\hline & 19 & 33.4867 & 29.0936 & 30.7787 & 30.8009 & 29.2327 & 0.5825 & 1.1321 & 2.1117 \\
\hline & 20 & 31.5817 & 28.7599 & 29.5814 & 29.4362 & 29.1383 & 0.4154 & 0.9974 & 3.4010 \\
\hline & 21 & 33.7065 & 29.9555 & 31.3749 & 31.2701 & 31.1676 & 0.4005 & 0.6440 & 3.0244 \\
\hline & 22 & 31.2330 & 28.0778 & 31.3142 & 30.0309 & 30.4576 & 0.3470 & 0.5555 & 2.0406 \\
\hline & 23 & 33.2233 & 29.6511 & 30.5988 & 30.5902 & 29.3866 & 0.1998 & 0.2890 & 2.1639 \\
\hline & 24 & 31.3467 & 28.6874 & 30.7960 & 30.8363 & 29.2576 & 0.2984 & -0.0572 & 3.5622 \\
\hline & 25 & 30.9871 & 27.7813 & 29.0544 & 30.2118 & 30.3741 & 0.4469 & 0.6921 & 3.6718 \\
\hline & 26 & 32.4456 & 29.6547 & 30.1920 & 30.8419 & 30.7761 & 0.4384 & 0.8383 & 1.7672 \\
\hline \multirow{8}{*}{ 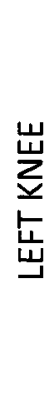 } & 1 & 32.1325 & 29.2579 & 31.0218 & 31.1872 & 31.3988 & 0.6037 & -0.7395 & 2.7100 \\
\hline & 2 & 31.4200 & 29.5205 & 30.5332 & 30.5110 & 30.4669 & 0.3355 & -0.0295 & 2.6400 \\
\hline & 3 & 31.8645 & 29.4685 & 30.5154 & 30.4742 & 29.8097 & 0.5694 & 0.2446 & 1.9134 \\
\hline & 4 & 28.1704 & 25.5611 & 27.3531 & 27.4409 & 27.6821 & 0.4235 & -1.1194 & 4.3429 \\
\hline & 5 & 31.0850 & 28.7592 & 29.8999 & 29.9170 & 29.8801 & 0.4175 & -0.1652 & 2.5133 \\
\hline & 6 & 31.0484 & 28.4754 & 30.0377 & 30.1751 & 30.3297 & 0.5569 & -0.5574 & 2.4235 \\
\hline & 7 & 31.1434 & 29.3693 & 30.4272 & 30.4474 & 30.4400 & 0.2915 & -0.5222 & 3.2053 \\
\hline & 8 & 31.6323 & 27.1840 & 29.2335 & 29.1704 & 27.1840 & 0.9486 & 0.0730 & 2.0262 \\
\hline
\end{tabular}




\begin{tabular}{|c|c|c|c|c|c|c|c|c|}
\hline 9 & 28.8878 & 26.9493 & 28.0124 & 28.0279 & 27.9979 & 0.3666 & -0.2073 & 2.7337 \\
\hline 10 & 31.7561 & 22.8927 & 29.5297 & 30.5878 & 30.4703 & 2.5207 & -1.5207 & 4.4411 \\
\hline 11 & 31.5372 & 29.4583 & 30.2717 & 30.2414 & 30.1457 & 0.3803 & 0.3999 & 2.7651 \\
\hline 12 & 32.7652 & 29.1966 & 30.9183 & 30.9437 & 31.0972 & 0.7165 & -0.0808 & 2.4402 \\
\hline 13 & 31.3485 & 28.1470 & 29.4361 & 29.4000 & 29.3555 & 0.4705 & 0.8634 & 4.9584 \\
\hline 14 & 32.1542 & 26.2905 & 30.8363 & 30.7960 & 30.7813 & 0.2984 & 0.6921 & 3.5622 \\
\hline 15 & 29.7629 & 23.4403 & 28.1458 & 28.0544 & 27.6874 & 0.4469 & 0.3473 & 3.6718 \\
\hline 16 & 31.7907 & 24.5952 & 30.2118 & 30.1920 & 29.2576 & 0.3006 & -0.0572 & 1.7672 \\
\hline 17 & 33.9736 & 30.3593 & 32.2559 & 32.2654 & 32.1106 & 0.3085 & -0.0345 & 2.6561 \\
\hline 18 & 33.8877 & 26.6206 & 32.7104 & 32.8055 & 33.0266 & 0.4384 & -0.2861 & 2.2536 \\
\hline 19 & 31.8101 & 28.7699 & 30.0147 & 29.9456 & 29.1135 & 0.2736 & 0.2649 & 1.9185 \\
\hline 20 & 30.0186 & 24.8746 & 302.9605 & 29.8419 & 29.1334 & 0.2262 & 0.4762 & 2.2989 \\
\hline 21 & 33.4628 & 25.6989 & 30.9359 & 30.8156 & 30.3741 & 0.3157 & 0.8383 & 3.3442 \\
\hline 22 & 34.0068 & 26.1724 & 31.8489 & 31.7761 & 31.7033 & 0.3753 & 0.2046 & 2.7792 \\
\hline 23 & 32.9113 & 28.7300 & 30.6915 & 30.7617 & 31.1481 & 0.5758 & -0.0658 & 3.0489 \\
\hline 24 & 31.8198 & 27.1576 & 31.1135 & 31.1969 & 31.3042 & 0.3357 & 0.0935 & 4.6589 \\
\hline 25 & 33.1034 & 28.6784 & 31.9898 & 31.9507 & 31.3481 & 0.4640 & 0.0027 & 5.8334 \\
\hline 26 & 31.6547 & 302.1483 & 30.5615 & 30.7568 & 30.8303 & 0.4943 & 0.0270 & 3.5678 \\
\hline GE & 33.0895 & 31.6711 & 31.8761 & 30.3275 & 30.2084 & 0.4556 & 0.0436 & 3.0268 \\
\hline
\end{tabular}


10.9. $I^{\text {st }}$ MTP Raw Data Measurement

Table 10-9: Raw Data Measurements Extracted from the Control Group's $1^{\text {st }}$ MTP Joint

\begin{tabular}{|c|c|c|c|c|c|c|c|c|c|}
\hline \multicolumn{10}{|c|}{ Control Group's 1st MTP Result } \\
\hline & & $\operatorname{Max}\left({ }^{\circ} \mathrm{C}\right)$ & $\operatorname{Min}\left({ }^{\circ} \mathrm{C}\right)$ & Mean $\left({ }^{\circ} \mathrm{C}\right)$ & Median ( $\left.{ }^{\circ} \mathrm{C}\right)$ & Mode $\left({ }^{\circ} \mathrm{C}\right)$ & STD & Skewness & Kurtosis \\
\hline \multirow{28}{*}{$\begin{array}{l}\text { ○ } \\
\text { 이 } \\
\text { 또 } \\
\frac{0}{x}\end{array}$} & 1 & 24.2016 & 22.6434 & 23.3888 & 23.3588 & 23.7264 & 0.3790 & 0.2001 & 1.9621 \\
\hline & 2 & 29.9253 & 27.1222 & 28.5628 & 28.5139 & 28.4466 & 0.6713 & 0.0898 & 2.1108 \\
\hline & 3 & 26.9480 & 25.2132 & 26.0436 & 26.0190 & 25.6590 & 0.4222 & 0.1218 & 2.0344 \\
\hline & 4 & 33.7582 & 31.4655 & 32.9004 & 32.9774 & 33.3219 & 0.5018 & -0.6832 & 2.8102 \\
\hline & 5 & 26.2339 & 25.1218 & 25.6764 & 25.6754 & 25.6601 & 0.2142 & 0.0060 & 2.5213 \\
\hline & 6 & 28.9754 & 27.4384 & 28.1223 & 28.1158 & 27.9882 & 0.3002 & 0.1470 & 2.2877 \\
\hline & 7 & 33.2137 & 32.0818 & 32.7524 & 32.7752 & 32.7824 & 0.2299 & -0.4045 & 2.6393 \\
\hline & 8 & 28.8562 & 28.2508 & 28.5517 & 28.5501 & 28.5352 & 0.1021 & 0.1399 & 2.8317 \\
\hline & 9 & $x$ & $x$ & $x$ & $x$ & $x$ & $x$ & $x$ & $x$ \\
\hline & 10 & 34.6685 & 30.8989 & 33.7408 & 33.7299 & 33.5458 & 0.4012 & 0.0994 & 2.0501 \\
\hline & 11 & 22.0583 & 21.2222 & 21.5729 & 21.5495 & 21.4857 & 0.1620 & 0.3376 & 2.4129 \\
\hline & 12 & 25.8675 & 23.9914 & 24.8913 & 24.8988 & 25.2303 & 0.4419 & 0.0611 & 2.0405 \\
\hline & 13 & 27.7345 & 27.1004 & 27.3934 & 27.3726 & 27.2819 & 0.1214 & 0.4158 & 2.4990 \\
\hline & 14 & 24.2160 & 23.2320 & 23.7378 & 23.7408 & 23.7876 & 0.2074 & 0.0400 & 2.1545 \\
\hline & 15 & 27.8754 & 27.1436 & 27.5567 & 27.5969 & 27.6496 & 0.1586 & -0.6367 & 2.6455 \\
\hline & 16 & 27.3491 & 26.2863 & 26.9402 & 26.9404 & 27.1752 & 0.2255 & -0.1575 & 1.8823 \\
\hline & 17 & 25.9588 & 24.6815 & 25.2809 & 25.2759 & 25.0215 & 0.2657 & 0.0986 & 2.3161 \\
\hline & 18 & 27.8228 & 26.8860 & 27.3251 & 27.3100 & 27.2344 & 0.2238 & 0.0835 & 1.8930 \\
\hline & 19 & 33.3786 & 32.0891 & 32.7542 & 32.8040 & 33.0414 & 0.3221 & -0.2416 & 1.7394 \\
\hline & 20 & 28.5651 & 27.7776 & 28.2692 & 28.3032 & 28.3482 & 0.1499 & -0.7922 & 3.0216 \\
\hline & 21 & 24.4965 & 23.2961 & 23.8487 & 23.8356 & 23.5857 & 0.2664 & 0.1915 & 2.1937 \\
\hline & 22 & 25.8371 & 24.7908 & 25.3368 & 25.3383 & 25.2998 & 0.2556 & -0.0645 & 1.9373 \\
\hline & 23 & 21.9154 & 21.2782 & 21.5809 & 21.5654 & 21.5176 & 0.1126 & 0.6268 & 3.3867 \\
\hline & 24 & 24.9297 & 23.8824 & 24.3769 & 24.3569 & 24.0771 & 0.2805 & 0.0798 & 1.5892 \\
\hline & 25 & 24.6350 & 23.8812 & 24.2393 & 24.2316 & 24.3481 & 0.1795 & 0.1230 & 1.9920 \\
\hline & 26 & 23.9046 & 22.7681 & 23.3283 & 23.3261 & 23.3496 & 0.2302 & 0.0467 & 2.4634 \\
\hline & 27 & 26.1552 & 25.2039 & 25.5717 & 25.5578 & 25.4348 & 0.1739 & 0.6779 & 3.5864 \\
\hline & 28 & 27.3566 & 26.3854 & 26.8314 & 26.8190 & 26.6062 & 0.2209 & 0.1953 & 1.9796 \\
\hline \multirow{6}{*}{ 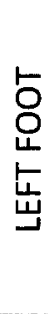 } & 1 & 25.4843 & 23.1392 & 24.1866 & 24.1861 & 24.2327 & 0.5211 & -0.0249 & 2.0158 \\
\hline & 2 & 29.6889 & 27.4623 & 28.4125 & 28.3792 & 27.9443 & 0.4825 & 0.3010 & 2.2026 \\
\hline & 3 & 27.2206 & 25.8200 & 26.4386 & 26.3931 & 26.3473 & 0.3134 & 0.2918 & 2.2012 \\
\hline & 4 & 33.2646 & 31.1884 & 32.4726 & 32.4588 & 33.0277 & 0.4459 & -0.1705 & 2.1729 \\
\hline & 5 & 26.3407 & 24.8671 & 25.6196 & 25.6371 & 25.9129 & 0.3230 & -0.1372 & 2.0839 \\
\hline & 6 & 28.9083 & 27.8679 & 28.3385 & 28.3145 & 28.3257 & 0.2062 & 0.3435 & 2.4165 \\
\hline
\end{tabular}




\begin{tabular}{|c|c|c|c|c|c|c|c|c|}
\hline 7 & 32.8688 & 31.5517 & 32.4744 & 32.5229 & 32.5157 & 0.2453 & -1.3251 & 4.7342 \\
\hline 8 & 28.8114 & 28.1158 & 28.3948 & 28.4006 & 28.4006 & 0.0995 & 0.1747 & 3.6495 \\
\hline 9 & $x$ & $x$ & $x$ & $x$ & $x$ & $x$ & $x$ & $x$ \\
\hline 10 & 33.6992 & 29.5121 & 32.1233 & 32.1381 & 33.9761 & 0.2286 & -0.0903 & 2.3287 \\
\hline 11 & 22.3513 & 21.3900 & 21.8073 & 21.7883 & 21.5813 & 0.2138 & 0.2209 & 2.1339 \\
\hline 12 & 25.8522 & 23.9681 & 25.0607 & 25.0994 & 25.4995 & 0.4224 & -0.3534 & 2.1321 \\
\hline 13 & 27.0776 & 26.6452 & 26.8682 & 26.8730 & 26.8882 & 0.0703 & -0.0365 & 3.0517 \\
\hline 14 & 24.2549 & 22.9728 & 23.6256 & 23.6470 & 23.6470 & 0.2918 & -0.0993 & 2.1302 \\
\hline 15 & 27.5742 & 26.8025 & 27.1143 & 27.1209 & 27.1815 & 0.1195 & 0.1021 & 2.9990 \\
\hline 16 & 27.3189 & 26.4388 & 26.8708 & 26.8645 & 26.7810 & 0.1514 & 0.3062 & 2.9335 \\
\hline 17 & 25.4681 & 24.5188 & 25.0134 & 25.0138 & 24.8671 & 0.2016 & -0.0670 & 2.2858 \\
\hline 18 & 27.6345 & 26.5516 & 27.0645 & 27.0414 & 26.9087 & 0.2351 & 0.2532 & 2.3102 \\
\hline 19 & 33.2997 & 32.5229 & 32.9702 & 32.9623 & 32.9120 & 0.1156 & -0.3894 & 4.4183 \\
\hline 20 & 28.0708 & 27.1890 & 27.6577 & 27.6647 & 27.7852 & 0.1656 & -0.2074 & 2.4583 \\
\hline 21 & 23.3509 & 22.3434 & 22.7790 & 22.7696 & 22.6356 & 0.2091 & 0.1781 & 2.4261 \\
\hline 22 & 25.5458 & 24.2871 & 24.8870 & 24.8603 & 24.8680 & 0.3004 & 0.2388 & 2.1397 \\
\hline 23 & 21.8519 & 21.1423 & 21.3989 & 21.3820 & 21.3580 & 0.1158 & 0.7371 & 3.6522 \\
\hline 24 & 24.8679 & 23.4919 & 24.0240 & 23.9798 & 23.7107 & 0.3234 & 0.4402 & 2.1872 \\
\hline 25 & 25.0987 & 24.2083 & 24.6763 & 24.6621 & 24.5111 & 0.2028 & 0.0435 & 2.0416 \\
\hline 26 & 23.5767 & 22.6420 & 23.0904 & 23.0986 & 23.0278 & 0.2076 & -0.0413 & 2.0682 \\
\hline 27 & 26.4603 & 25.5655 & 25.9236 & 25.9105 & 25.9258 & 0.1507 & 0.4833 & 3.3473 \\
\hline 28 & 27.0768 & 26.0420 & 26.5421 & 26.5149 & 26.4464 & 0.2354 & 0.1522 & 2.1941 \\
\hline GE & 26.9517 & 25.7686 & 26.3566 & 26.3530 & 26.3430 & 0.2536 & 0.0407 & 2.4869 \\
\hline
\end{tabular}


10.10. $1^{\text {st }}$ MTP Raw Data Measurement

Table 10-10: Raw Data Measurements Extracted from the Patient Group's $1^{\text {st }}$ MTP Joint

\begin{tabular}{|c|c|c|c|c|c|c|c|c|c|}
\hline \multicolumn{10}{|c|}{ Patient Group's 1st MTP Result } \\
\hline & & $\operatorname{Max}\left({ }^{\circ} \mathrm{C}\right)$ & $\operatorname{Min}\left({ }^{\circ} \mathrm{C}\right)$ & Mean $\left({ }^{\circ} \mathrm{C}\right)$ & Median $\left({ }^{\circ} \mathrm{C}\right)$ & Mode $\left({ }^{\circ} \mathrm{C}\right)$ & STD & Skewness & Kurtosis \\
\hline \multirow{21}{*}{$\begin{array}{l}\text { 응 } \\
\text { 오 } \\
\text { 도 } \\
\frac{1}{x}\end{array}$} & 1 & 26.4603 & 25.6729 & 26.4603 & 26.1322 & 26.2391 & 0.1911 & -0.3768 & 2.0682 \\
\hline & 2 & 30.5625 & 29.2529 & 29.6008 & 29.5502 & 29.4463 & 0.2022 & 1.2153 & 4.9357 \\
\hline & 3 & 26.8837 & 26.0366 & 26.4304 & 26.4419 & 26.5029 & 0.1837 & 0.0046 & 2.0453 \\
\hline & 4 & 25.1227 & 23.3039 & 23.9799 & 23.9214 & 23.5075 & 0.4114 & 0.4806 & 2.2887 \\
\hline & 5 & 25.9717 & 24.8412 & 25.3942 & 25.3887 & 25.3810 & 0.2169 & 0.0664 & 2.6271 \\
\hline & 6 & 25.4809 & 23.7768 & 24.6428 & 24.7019 & 25.1191 & 0.4377 & -0.2073 & 1.7482 \\
\hline & 7 & 28.5427 & 27.8153 & 28.2277 & 28.2508 & 28.3332 & 0.1461 & -0.4551 & 2.3864 \\
\hline & 8 & 30.9908 & 29.1630 & 30.2475 & 30.2794 & 30.2352 & 0.3692 & -0.3692 & 2.8555 \\
\hline & 9 & $x$ & $x$ & $x$ & $x$ & $x$ & $x$ & $x$ & $x$ \\
\hline & 10 & $x$ & $x$ & $\mathrm{x}$ & $x$ & $x$ & $\mathrm{x}$ & $x$ & $x$ \\
\hline & 11 & 26.3459 & 25.2655 & 25.7925 & 25.7879 & 25.8492 & 0.2282 & 0.0160 & 2.2189 \\
\hline & 12 & 26.2914 & 24.7709 & 25.3989 & 25.3647 & 25.1183 & 0.3377 & 0.2952 & 2.1530 \\
\hline & 13 & 26.8797 & 24.6494 & 25.6749 & 25.6130 & 25.0590 & 0.5891 & 0.1972 & 1.7888 \\
\hline & 14 & 26.3078 & 25.3810 & 25.7871 & 25.7496 & 25.9181 & 0.2165 & 0.2933 & 2.2051 \\
\hline & 15 & 34.1045 & 31.7189 & 33.1312 & 33.1374 & 33.1374 & 0.5604 & -0.1998 & 2.1249 \\
\hline & 16 & 27.0660 & 26.4953 & 26.7977 & 26.8077 & 26.8609 & 0.1110 & -0.1727 & 2.6423 \\
\hline & 17 & 24.8217 & 23.3196 & 24.0627 & 24.0616 & 24.1549 & 0.3542 & 0.0523 & 1.9894 \\
\hline & 18 & 26.3078 & 25.0574 & 25.7465 & 25.7534 & 25.5040 & 0.2773 & -0.0145 & 2.0693 \\
\hline & 24 & 26.6201 & 25.7343 & 26.2478 & 26.2620 & 26.2544 & 0.1788 & -0.3940 & 2.5350 \\
\hline & 25 & 26.4134 & 25.2262 & 25.7188 & 25.6949 & 25.7869 & 0.2530 & 0.3008 & 2.3521 \\
\hline & 26 & 26.3702 & 25.2671 & 25.8737 & 25.8660 & 25.9425 & 0.2102 & -0.0263 & 2.6502 \\
\hline \multirow{8}{*}{ 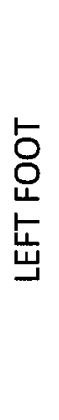 } & 1 & 27.6345 & 26.7873 & 27.2436 & 27.2420 & 27.3478 & 0.1709 & -0.1159 & 2.4140 \\
\hline & 2 & 31.7189 & 31.0177 & 31.4479 & 31.4565 & 31.5732 & 0.1193 & -0.3026 & 2.9753 \\
\hline & 3 & 27.5431 & 26.7468 & 27.1300 & 27.1229 & 26.9521 & 0.1841 & 0.1497 & 2.0413 \\
\hline & 4 & 24.8603 & 23.9292 & 24.3829 & 24.3725 & 24.1705 & 0.1971 & 0.1383 & 1.9114 \\
\hline & 5 & 25.4809 & 24.6710 & 25.1092 & 25.1500 & 25.2039 & 0.1820 & -0.3598 & 2.1606 \\
\hline & 6 & 25.4656 & 24.1743 & 24.8504 & 24.8876 & 25.0651 & 0.3018 & -0.2219 & 2.0462 \\
\hline & 7 & 28.7592 & 28.0182 & 28.4228 & 28.4455 & 28.4455 & 0.1503 & -0.4090 & 2.4912 \\
\hline & 8 & 25.4613 & 23.8356 & 24.5908 & 24.5741 & 24.3414 & 0.3423 & 0.1448 & 2.2140 \\
\hline
\end{tabular}




\begin{tabular}{|c|c|c|c|c|c|c|c|c|}
\hline 9 & $x$ & $x$ & $x$ & $x$ & $x$ & $x$ & $x$ & $x$ \\
\hline 10 & $x$ & $x$ & $x$ & $x$ & $x$ & $\mathrm{x}$ & $x$ & $x$ \\
\hline 11 & 271588 & 262391 & 266229 & 265897 & 264983 & 01894 & 04518 & 22669 \\
\hline 12 & 263448 & 247631 & 255463 & 255338 & 256259 & 03150 & 00642 & 25641 \\
\hline 13 & 285139 & 254210 & 269471 & 270010 & 275076 & 07388 & -00740 & 19913 \\
\hline 14 & 271285 & 257419 & 265471 & 265516 & 266277 & 03050 & -02614 & 25147 \\
\hline 15 & 314930 & 292827 & 306247 & 307242 & 310250 & 05328 & -04743 & 22414 \\
\hline 16 & 283263 & 274373 & 279222 & 279354 & 279279 & 01752 & -03062 & 26079 \\
\hline 17 & 246670 & 230528 & 238370 & 238317 & 237888 & 03380 & 00212 & 22032 \\
\hline 18 & 256883 & 243763 & 250937 & 251423 & 252424 & 03057 & -02432 & 18982 \\
\hline 19 & 254502 & 241898 & 248086 & 248335 & 250189 & 02654 & -02260 & 22090 \\
\hline 20 & 284604 & 274762 & 279084 & 278980 & 278905 & 02127 & 01506 & 22725 \\
\hline 21 & 286342 & 259519 & 273330 & 273579 & 270932 & 05136 & -00993 & 30428 \\
\hline 22 & 272445 & 256302 & 265415 & 265469 & 267900 & 03370 & -02349 & 23808 \\
\hline 23 & 293555 & 282369 & 289317 & 289504 & 289392 & 02191 & -04523 & 25086 \\
\hline 24 & 268708 & 257189 & 264063 & 264222 & 261781 & 02586 & -02396 & 21035 \\
\hline 25 & 259247 & 252570 & 255729 & 255722 & 255031 & 01212 & -00636 & 25903 \\
\hline 26 & 259655 & 241604 & 254035 & 254671 & 256897 & 03650 & -09090 & 34045 \\
\hline $\mathrm{GE}$ & 27.3513 & 26.0452 & 26.7467 & 26.7239 & 26.7116 & 0.2848 & -0.0688 & 2.3834 \\
\hline
\end{tabular}


11. APPENDIX E_Decision Trees

111 Decision Tree(classification) Results of presence or absence of RA

Table 11-1: Min/Max and Median/Max Decision Tree result (Using 13 patients ad 16 control subjects)

\begin{tabular}{|c|l|r|r|r|r|r|}
\hline \multicolumn{2}{|c|}{ DT\# } & 1 & 2 & 3 & 4 & 5 \\
\hline \multirow{3}{*}{ Train } & Sensitivity & 76.4 & 71.2 & 75.4 & 77.3 & 77.3 \\
& Specificity & 60.2 & 52.5 & 56.8 & 58.3 & 60.7 \\
& CCR & 65.3 & 59.8 & 63.1 & 64.8 & 66.0 \\
\hline \multirow{3}{*}{ Test } & Sensitivity & 60.8 & 63.4 & 65.2 & 65.2 & 63.6 \\
& Specificity & 48.5 & 50.8 & 52.3 & 52.3 & 51.2 \\
& CCR & 51.7 & 54.1 & 56.8 & 56.8 & 54.7 \\
\hline
\end{tabular}

Table 11-2: Mode/Min and Median/Min Decision Tree result (Using 13 patients and 16 control subjects)

\begin{tabular}{|c|l|r|r|r|r|r|}
\hline \multicolumn{2}{|l|}{ DT\# } & 1 & 2 & 3 & 4 & 5 \\
\hline \multirow{4}{*}{ Train } & Sensitivity & 70.8 & 67.1 & 64.3 & 59.6 & 59.6 \\
& Specificity & 63.8 & 60.7 & 59.2 & 55.1 & 54.9 \\
& CCR & 66.3 & 62.5 & 60.8 & 56.5 & 56.1 \\
\hline \multirow{5}{*}{ Test } & Sensitivity & 53.4 & 50.8 & 52.1 & 48.7 & 49.2 \\
& Specificity & 70.0 & 65.4 & 65.4 & 60.8 & 69.3 \\
& CCR & 54.7 & 50.6 & 52.5 & 48.8 & 49.3 \\
\hline
\end{tabular}


Table 11-3: Mode/Min and Mean/Min Decision Tree result (Using 13 patients and 16 control subjects)

\begin{tabular}{|c|l|r|r|r|r|r|}
\hline \multicolumn{2}{|l|}{ DT\# } & 1 & 2 & 3 & 4 & 5 \\
\hline \multirow{4}{*}{ Train } & Sensitivity & 68.9 & 74.2 & 69.3 & 70.0 & 75.4 \\
& Specificity & 56.3 & 58.8 & 54.1 & 55.9 & 61.7 \\
& CCR & 58.6 & 62.5 & 59.7 & 59.9 & 64.6 \\
\hline \multirow{4}{*}{ Test } & Sensitivity & 45.2 & 44.6 & 44.6 & 42.2 & 45.2 \\
& Specificity & 50.6 & 46.5 & 41.9 & 51.6 & 55.0 \\
& CCR & 47.9 & 45.7 & 42.3 & 46.7 & 51.1 \\
\hline
\end{tabular}

Table 11-4: Mode/Max and Median/Max Decision Tree result (Using 13 patients and 16 control subjects)

\begin{tabular}{|c|c|c|c|c|c|c|}
\hline DT\# & & 1 & 2 & 3 & 4 & 5 \\
\hline \multirow{3}{*}{ Train } & Sensitivity & 72.3 & 66.4 & 70.9 & 67.1 & 70.9 \\
\hline & Specificity & 69.2 & 62.4 & 58.2 & 56.7 & 62.4 \\
\hline & CCR & 69.8 & 63.4 & 63.6 & 60.9 & 65.7 \\
\hline \multirow{3}{*}{ Test } & Sensitivity & 48.1 & 48.1 & 44.3 & 45.9 & 47.1 \\
\hline & Specificity & 45.7 & 59.3 & 58.8 & 54.6 & 55.3 \\
\hline & CCR & 47.9 & 54.7 & 52.6 & 51.3 & 52.2 \\
\hline
\end{tabular}


Table 11-5: Min/Max and Median/Min Decision Tree result (Using 13 patients and 16 control subjects)

\begin{tabular}{|c|l|r|r|r|r|r|}
\hline \multicolumn{2}{|c|}{ DT\# } & 1 & $\mathbf{2}$ & 3 & 4 & 5 \\
\hline \multirow{3}{*}{ Train } & Sensitivity & 82.5 & 82.5 & 80.4 & 78.5 & 78.5 \\
& Specificity & 79.0 & 74.5 & 77.8 & 72.4 & 72.4 \\
& CCR & 79.8 & 77.2 & 78.1 & 74.5 & 74.5 \\
\hline \multirow{3}{*}{ Test } & Sensitivity & 69.0 & 69.0 & 67.1 & 65.4 & 60.3 \\
& Specificity & 79.4 & 76.1 & 72.5 & 62.8 & 62.8 \\
& SCCR & 73.2 & 71.6 & 68.8 & 63.1 & 60.6 \\
\hline
\end{tabular}

Table 11-6: Min/Max and Mean/Min Decision Tree result (Using 13 patients and 16 control subjects)

\begin{tabular}{|c|l|r|r|r|r|r|}
\hline \multicolumn{2}{|l|}{ DT\# } & 1 & 2 & 3 & 4 & 5 \\
\hline \multirow{4}{*}{ Train } & Sensitivity & 82.6 & 75.4 & 80.3 & 79.8 & 79.8 \\
& Specificity & 77.6 & 65.4 & 78.6 & 70.2 & 70.2 \\
& CCR & 78.1 & 68.4 & 77.5 & 73.0 & 73.0 \\
\hline \multirow{5}{*}{ Test } & Sensitivity & 66.4 & 58.5 & 62.5 & 66.4 & 66.4 \\
& Specificity & 57.9 & 50.5 & 58.6 & 60.1 & 63.3 \\
& CCR & 60.2 & 52.5 & 58.6 & 61.3 & 62.9 \\
\hline
\end{tabular}


Table 11-7: Median/Max and Mode/Min Decision Tree result (Using 13 patients and 16 control subjects)

\begin{tabular}{|l|l|r|r|r|r|r|}
\hline \multicolumn{2}{|l|}{ DT\# } & 1 & 2 & 3 & 4 & 5 \\
\hline \multirow{4}{*}{ Train } & Sensitivity & 85.2 & 73.4 & 82.6 & 80.3 & 80.3 \\
& Specificity & 80.2 & 68.8 & 78.3 & 75.4 & 75.4 \\
& CCR & 80.2 & 72.1 & 79.5 & 76.9 & 76.9 \\
\hline \multirow{5}{*}{ Test } & Sensitivity & 70.2 & 65.4 & 70.2 & 69.4 & 69.4 \\
& Specificity & 75.5 & 58.7 & 77.1 & 68.9 & 65.6 \\
& CCR & 71.9 & 61.1 & 72.7 & 68.2 & 66.5 \\
\hline
\end{tabular}

Table 11-8: Mean/Max and Min/Max Decision Tree result (Using 13 patients and 16 control subjects)

\begin{tabular}{|l|l|r|r|r|r|r|}
\hline \multicolumn{2}{|l|}{ DT\# } & 1 & 2 & 3 & 4 & 5 \\
\hline \multirow{4}{*}{ Train } & Sensitivity & 79.3 & 68.7 & 77.5 & 73.2 & 70.4 \\
& Specificity & 70.0 & 63.1 & 75.7 & 63.6 & 69.9 \\
& CCR & 69.7 & 60.9 & 71.6 & 63.4 & 65.2 \\
\hline \multirow{4}{*}{ Test } & Sensitivity & 50.3 & 50.3 & 45.6 & 43.2 & 49.6 \\
& Specificity & 71.1 & 65.4 & 50.3 & 49.7 & 51.7 \\
& CCR & 55.7 & 52.9 & 43.0 & 41.5 & 45.7 \\
\hline
\end{tabular}


Table 11-9: Min/Max and Mode/Min Decision Tree result (Using 13 patients and 16 control subjects)

\begin{tabular}{|l|l|r|r|r|r|r|}
\hline \multicolumn{2}{|l|}{ DT\# } & 1 & 2 & 3 & 4 & 5 \\
\hline \multirow{4}{*}{ Train } & Sensitivity & 81.5 & 76.4 & 80.3 & 70.0 & 76.9 \\
& Specificity & 70.1 & 65.6 & 73.7 & 69.3 & 72.3 \\
& CCR & 70.8 & 66.0 & 72.3 & 64.6 & 69.6 \\
\hline \multirow{4}{*}{ Test } & Sensitivity & 60.3 & 61.2 & 59.8 & 56.1 & 59.8 \\
& Specificity & 45.6 & 45.6 & 42.6 & 47.5 & 40.4 \\
& CCR & 49.9 & 50.4 & 47.2 & 47.8 & 46.1 \\
\hline
\end{tabular}

Table 11-10: Min/Max and Mode/Min Decision Tree result (Using 13 patients and 16 control subjects)

\begin{tabular}{|c|l|r|r|r|r|r|}
\hline \multicolumn{2}{|l|}{ DT\# } & 1 & 2 & 3 & 4 & 5 \\
\hline \multirow{4}{*}{ Train } & Sensitivity & 86.5 & 75.4 & 85.9 & 85.9 & 84.1 \\
& Specificity & 75.2 & 73.1 & 70.0 & 71.4 & 73.6 \\
& CCR & 75.2 & 69.3 & 73.0 & 74.1 & 73.9 \\
\hline \multirow{4}{*}{ Test } & Sensitivity & 69.2 & 69.2 & 64.8 & 60.5 & 60.5 \\
& Specificity & 54.4 & 48.0 & 50.1 & 53.2 & 50.3 \\
& CCR & 56.8 & 53.6 & 52.5 & 51.9 & 50.4 \\
\hline
\end{tabular}


Table 11-11: Mode/Max and Mean/Max Decision Tree result (Using 13 patients and 16 control subjects)

\begin{tabular}{|c|l|r|r|r|r|r|}
\hline \multicolumn{1}{|c|}{ DT\# } & 1 & 2 & 3 & 4 & 5 \\
\hline \multirow{3}{*}{ Train } & Sensitivity & 92.4 & 90.1 & 88.7 & 88.7 & 85.6 \\
& Specificity & 83.3 & 76.3 & 79.4 & 79.4 & 80.5 \\
& CCR & 83.9 & 79.2 & 79.1 & 79.1 & 80.1 \\
\hline \multirow{3}{*}{ Test } & Sensitivity & 70.6 & 67.4 & 71.6 & 71.6 & 72.2 \\
& Specificity & 63.3 & 60.2 & 65.1 & 62.0 & 79.1 \\
& SpCR & 63.0 & 59.8 & 64.4 & 62.8 & 71.7 \\
\hline
\end{tabular}

Table 11-12: Min/Max and Median/Max Decision Tree result (Using 26 patients ad 26 control subjects)

\begin{tabular}{|c|c|c|c|c|c|c|}
\hline DT\# & & 1 & 2 & 3 & 4 & 5 \\
\hline \multirow{3}{*}{ Train } & Sensitivity & 100 & 100 & 96.1 & 88.9 & 88.9 \\
\hline & Specificity & 92.7 & 88.7 & 90.2 & 83.5 & 83.5 \\
\hline & CCR & 94.4 & 90.5 & 91.2 & 82.2 & 82.2 \\
\hline \multirow{3}{*}{ Test } & Sensitivity & 93.4 & 90.1 & 87.4 & 85.3 & 85.3 \\
\hline & Specificity & 81.1 & 87.3 & 80.3 & 79.4 & 82.1 \\
\hline & CCR & 82.3 & 83.7 & 78.9 & 77.4 & 78.7 \\
\hline
\end{tabular}


Table 11-13: Mode/Min and Median/Min Decision Tree result (Using 26 patients and 26 control subjects)

\begin{tabular}{|c|l|r|r|r|r|r|}
\hline \multicolumn{2}{|c|}{ DT\# } & 1 & 2 & 3 & 4 & 5 \\
\hline \multirow{3}{*}{ Train } & Sensitivity & 100 & 96.8 & 89.6 & 90.1 & 98.6 \\
& Specificity & 92.5 & 90.7 & 82.5 & 84.6 & 87.5 \\
& CCR & 94.1 & 90.8 & 83.1 & 84.4 & 90.1 \\
\hline \multirow{3}{*}{ Test } & Sensitivity & 89.6 & 90.9 & 94.1 & 92.2 & 92.2 \\
& Specificity & 78.9 & 86.5 & 92.3 & 90.6 & 86.1 \\
& SpCR & 81.3 & 85.7 & 90.2 & 88.4 & 83.2 \\
\hline
\end{tabular}

Table 11-14: Mode/Min and Mean/Min Decision Tree result (Using 26 patients and 26 control subjects)

\begin{tabular}{|r|r|r|r|r|r|r|}
\hline \multicolumn{2}{|c|}{ DT\# } & 1 & 2 & 3 & 4 & 5 \\
\hline \multirow{3}{*}{ Train } & Sensitivity & 80.0 & 75.9 & 78.6 & 73.1 & 78.6 \\
& Specificity & 77.1 & 72.1 & 69.8 & 68.1 & 73.4 \\
& CCR & 77.6 & 73.0 & 73.2 & 69.6 & 75.0 \\
\hline \multirow{3}{*}{ Test } & Sensitivity & 79.2 & 72.2 & 73.9 & 72.2 & 80.0 \\
& Specificity & 74.0 & 69.2 & 64.1 & 69.2 & 76.3 \\
& SPR & 75.6 & 69.7 & 68.0 & 69.7 & 77.2 \\
\hline
\end{tabular}


Table 11-15: Mode/Max and Median/Max Decision Tree result (Using 26 patients and 26 control subjects)

\begin{tabular}{|r|l|r|r|r|r|r|}
\hline \multicolumn{2}{|c|}{ DT\# } & 1 & 2 & 3 & 4 & 5 \\
\hline \multirow{3}{*}{ Train } & Sensitivity & 88.3 & 73.9 & 80.7 & 80.7 & 80.7 \\
& Specificity & 75.6 & 67.1 & 77.3 & 70.6 & 75.6 \\
& CCR & 76.3 & 65.5 & 74.0 & 70.7 & 73.2 \\
\hline \multirow{6}{*}{ Test } & Sensitivity & 71.2 & 69.9 & 75.4 & 70.8 & 69.9 \\
& Specificity & 70.5 & 60.7 & 70.2 & 62.4 & 65.6 \\
& SCR & 65.9 & 60.3 & 67.8 & 61.6 & 62.8 \\
\hline
\end{tabular}

Table 11-16: Min/Max and Median/Min Decision Tree result (Using 26 patients and 26 control subjects)

\begin{tabular}{|r|r|r|r|r|r|r|}
\hline \multicolumn{2}{|l|}{ DT\# } & 1 & 2 & 3 & 4 & 5 \\
\hline \multirow{4}{*}{ Train } & Sensitivity & 100 & 100 & 100 & 90.6 & 87.7 \\
& Specificity & 85.4 & 82.7 & 78.1 & 86.6 & 77.5 \\
& CCR & 90.7 & 87.4 & 84.1 & 85.6 & 78.6 \\
\hline \multirow{4}{*}{ Test } & Sensitivity & 94.2 & 91.3 & 95.1 & 91.3 & 86.1 \\
& Specificity & 89.9 & 83.5 & 92.3 & 84.9 & 72.5 \\
& CCR & 89.1 & 84.4 & 90.7 & 85.1 & 75.3 \\
\hline
\end{tabular}


Table 11-17: Min/Max and Mean/Min Decision Tree result (Using 26 patients and 26 control subjects)

\begin{tabular}{|c|l|r|r|r|r|r|}
\hline \multicolumn{2}{|c|}{ DT\# } & 1 & 2 & 3 & 4 & 5 \\
\hline \multirow{3}{*}{ Train } & Sensitivity & 89.6 & 80.6 & 84.8 & 84.8 & 84.8 \\
& Specificity & 79.1 & 78.9 & 70.1 & 68.3 & 65.8 \\
& CCR & 79.4 & 74.8 & 72.5 & 71.6 & 70.3 \\
\hline \multirow{3}{*}{ Test } & Sensitivity & 65.4 & 58.7 & 63.3 & 58.7 & 58.7 \\
& Specificity & 60.2 & 54.5 & 58.6 & 55.1 & 55.1 \\
& SeCR & 59.8 & 53.6 & 57.0 & 53.9 & 53.9 \\
\hline
\end{tabular}

Table 11-18: Median/Max and Mode/Min Decision Tree result (Using 26 patients and 26 control subjects)

\begin{tabular}{|c|l|r|r|r|r|r|}
\hline \multicolumn{2}{|c|}{ DT\# } & 1 & 2 & 3 & 4 & 5 \\
\hline \multirow{4}{*}{ Train } & Sensitivity & 80.0 & 77.5 & 79.0 & 78.1 & 79.0 \\
& Specificity & 72.6 & 70.2 & 75.3 & 72.2 & 75.3 \\
& CCR & 72.3 & 69.9 & 71.2 & 71.2 & 73.3 \\
\hline \multirow{3}{*}{ Test } & Sensitivity & 79.2 & 79.2 & 77.7 & 79.2 & 80.0 \\
\cline { 3 - 7 } & Specificity & 72.2 & 66.6 & 71.0 & 80.1 & 81.4 \\
& SCCR & 71.7 & 68.9 & 70.4 & 75.7 & 80.4 \\
\hline
\end{tabular}


Table 11-19: Median/Max and Mode/Min Decision Tree result (Using 26 patients and 26 control subjects)

\begin{tabular}{|c|l|r|r|r|r|r|}
\hline \multicolumn{2}{|c|}{ DT\# } & 1 & 2 & 3 & 4 & 5 \\
\hline \multirow{3}{*}{ Train } & Sensitivity & 95.2 & 78.9 & 86.4 & 77.1 & 83.6 \\
& Specificity & 82.9 & 80.2 & 78.3 & 72.3 & 72.3 \\
& CCR & 83.1 & 73.6 & 76.4 & 67.7 & 71.0 \\
\hline \multirow{3}{*}{ Test } & Sensitivity & 75.4 & 78.4 & 78.4 & 79.4 & 79.4 \\
& Specificity & 68.3 & 66.1 & 74.2 & 70.0 & 77.2 \\
& SCR & 64.9 & 65.3 & 68.3 & 67.7 & 70.3 \\
\hline
\end{tabular}

Table 11-20: Min/Max and Mode/Min Decision Tree result (Using 26 patients and 26 control subjects)

\begin{tabular}{|c|l|r|r|r|r|r|}
\hline \multicolumn{2}{|l|}{ DT\# } & 1 & 2 & 3 & 4 & 5 \\
\hline \multirow{4}{*}{ Train } & Sensitivity & 69.1 & 54.5 & 54.5 & 70.0 & 62.3 \\
& Specificity & 56.9 & 50.3 & 48.3 & 62.5 & 55.4 \\
& CCR & 60.2 & 49.4 & 47.4 & 62.4 & 54.9 \\
\hline \multirow{4}{*}{ Test } & Sensitivity & 48.2 & 46.5 & 46.5 & 50.0 & 45.2 \\
& Specificity & 44.7 & 33.2 & 38.9 & 50.0 & 39.1 \\
& CCR & 43.5 & 35.9 & 38.7 & 50.0 & 38.4 \\
\hline
\end{tabular}


Table 11-21: Mode/Min and Median/Max Decision Tree result (Using 26 patients and 26 control subjects)

\begin{tabular}{|c|l|r|r|r|r|r|}
\hline \multicolumn{2}{|l|}{ DT \# } & 1 & 2 & 3 & 4 & 5 \\
\hline \multirow{4}{*}{ Train } & Sensitivity & 78.6 & 78.6 & 65.9 & 78.6 & 75.4 \\
& Specificity & 66.1 & 59.9 & 60.6 & 70.2 & 58.1 \\
& CCR & 66.1 & 64.3 & 60.3 & 70.0 & 61.8 \\
\hline \multirow{4}{*}{ Test } & Sensitivity & 58.3 & 60.1 & 60.1 & 60.1 & 55.7 \\
& Specificity & 48.9 & 62.4 & 52.2 & 52.2 & 50.3 \\
& CCR & 50.6 & 57.3 & 52.2 & 52.2 & 49.0 \\
\hline
\end{tabular}

Table 11-22: Mode/Max and Mean/Max Decision Tree result (Using 26 patients and 26 control subjects)

\begin{tabular}{|c|l|r|r|r|r|r|}
\hline \multicolumn{2}{|l|}{ DT\# } & 1 & 2 & 3 & 4 & 5 \\
\hline \multirow{4}{*}{ Train } & Sensitivity & 100 & 90.5 & 88.3 & 94.4 & 83.3 \\
& Specificity & 86.7 & 85.5 & 73.3 & 86.0 & 79.3 \\
& CCR & 86.0 & 81.1 & 77.4 & 83.0 & 79.1 \\
\hline \multirow{4}{*}{ Test } & Sensitivity & 89.1 & 89.1 & 78.3 & 78.3 & 81.4 \\
\cline { 2 - 7 } & Specificity & 74.9 & 87.2 & 63.3 & 67.1 & 67.1 \\
& CCR & 72.9 & 79.5 & 66.1 & 69.3 & 73.3 \\
\hline
\end{tabular}




\subsection{Decision Tree results for elimination of Variables (Joints)}

\subsubsection{Results for Knee, $2^{\text {nd }} M C P$ and $3^{\text {rd }} M C P$ combination}

Table 11-23: Mode/Min and Median/Min Decision Tree result (Using 26 patients and 26 control subjects)

\begin{tabular}{|r|l|r|r|r|r|r|}
\hline \multicolumn{2}{|l|}{ DT\# } & 1 & 2 & 3 & 4 & 5 \\
\hline \multirow{4}{*}{ Train } & Sensitivity & 100 & 100 & 96.4 & 87.9 & 88.6 \\
& Specificity & 83.9 & 68.7 & 86.5 & 77.1 & 77.1 \\
& CCR & 80.0 & 78.4 & 84.9 & 77.1 & 76.5 \\
\hline \multirow{5}{*}{ Test } & Sensitivity & 89.1 & 89.1 & 78.3 & 81.6 & 81.6 \\
& Specificity & 74.9 & 85.2 & 68.3 & 74.9 & 74.9 \\
& CCR & 80.6 & 84.4 & 63.3 & 72.1 & 72.4 \\
\hline
\end{tabular}

Table 11-24: Mode/Min and Median/Max Decision Tree result (Using 26 patients and 26 control subjects)

\begin{tabular}{|c|l|r|r|r|r|r|}
\hline \multicolumn{2}{|l|}{ DT\# } & $\mathbf{1}$ & $\mathbf{2}$ & $\mathbf{3}$ & $\mathbf{4}$ & $\mathbf{5}$ \\
\hline \multirow{4}{*}{ Train } & Sensitivity & 100 & 100 & 87.5 & 94.0 & 94.0 \\
& Specificity & 86.5 & 84.9 & 85.5 & 88.6 & 88.6 \\
& CCR & 85.2 & 83.6 & 81.6 & 86.7 & 85.5 \\
\hline \multirow{4}{*}{ Test } & Sensitivity & 90.0 & 90.0 & 90.0 & 87.2 & 87.2 \\
& Specificity & 78.6 & 88.2 & 84.6 & 86.3 & 86.3 \\
& CCR & 76.5 & 80.2 & 76.5 & 78.3 & 77.5 \\
\hline
\end{tabular}


Table 11-25: Mode/Max and Mean/Max Decision Tree result (Using 26 patients and 26 control subjects)

\begin{tabular}{|l|l|r|r|r|r|r|}
\hline \multicolumn{2}{|l|}{ DT\# } & 1 & 2 & 3 & 4 & 5 \\
\hline \multirow{4}{*}{ Train } & Sensitivity & 100 & 100 & 100 & 91.1 & 91.1 \\
& Specificity & 89.3 & 82.6 & 75.4 & 82.6 & 74.3 \\
& CCR & 90.2 & 86.7 & 79.5 & 78.2 & 81.2 \\
\hline \multirow{4}{*}{ Test } & Sensitivity & 92.3 & 80.6 & 83.1 & 83.1 & 80.6 \\
& Specificity & 85.0 & 77.4 & 82.5 & 80.6 & 77.4 \\
& CCR & 85.0 & 77.4 & 81.7 & 76.3 & 76.4 \\
\hline
\end{tabular}

Table 11-26: Mean/Max and Mode/Min Decision Tree result (Using 26 patients and 26 control subjects)

\begin{tabular}{|c|l|r|r|r|r|r|}
\hline \multicolumn{1}{|c|}{ DT\# } & 1 & 2 & 3 & 4 & 5 \\
\hline \multirow{4}{*}{ Train } & Sensitivity & 95.6 & 95.6 & 86.7 & 84.3 & 88.6 \\
& Specificity & 83.9 & 68.7 & 86.5 & 77.1 & 77.1 \\
& CCR & 81.6 & 75.2 & 80.4 & 79.3 & 79.3 \\
\hline \multirow{3}{*}{ Test } & Sensitivity & 78.6 & 78.6 & 80.3 & 80.3 & 78.8 \\
& Specificity & 77.3 & 76.8 & 78.0 & 75.2 & 75.2 \\
& CCR & 72.6 & 70.4 & 77.9 & 73.6 & 70.7 \\
\hline
\end{tabular}




\subsubsection{Result for $3^{\text {rd }} M C P$}

Table 11-27: Min/Max and Median/Max Decision Tree result (Using 26 patients and 26 control subjects)

\begin{tabular}{|r|l|r|r|r|r|r|}
\hline \multicolumn{1}{|c|}{ DT\# } & 1 & 2 & 3 & 4 & $\mathbf{5}$ \\
\hline \multirow{4}{*}{ Train } & Sensitivity & 100 & 85.4 & 85.4 & 91.6 & 91.6 \\
& Specificity & 89.9 & 80.6 & 83.4 & 87.5 & 87.5 \\
& CCR & 87.4 & 78.9 & 76.5 & 78.9 & 80.2 \\
\hline \multirow{4}{*}{ Test } & Sensitivity & 88.5 & 85.5 & 85.5 & 75.8 & 88.1 \\
& Specificity & 82.2 & 78.6 & 83.4 & 68.6 & 86.4 \\
& CCR & 79.9 & 77.1 & 81.1 & 65.3 & 83.5 \\
\hline
\end{tabular}

Table 11-28: Min/Max and Mean/Min Decision Tree result (Using 26 patients and 26 control subjects)

\begin{tabular}{|c|l|r|r|r|r|r|}
\hline \multicolumn{2}{|l|}{ DT\# } & 1 & 2 & 3 & 4 & 5 \\
\hline \multirow{4}{*}{ Train } & Sensitivity & 100 & 100 & 100 & 97.5 & 94.6 \\
& Specificity & 89.4 & 80.6 & 79.9 & 86.7 & 85.2 \\
& CCR & 86.9 & 82.0 & 81.5 & 78.7 & 79.3 \\
\hline \multirow{5}{*}{ Test } & Sensitivity & 90.0 & 90.0 & 90.0 & 90.0 & 90.0 \\
& Specificity & 86.8 & 78.7 & 90.0 & 76.3 & 77.2 \\
& CCR & 84.4 & 78.7 & 88.2 & 79.1 & 78.6 \\
\hline
\end{tabular}




\subsection{Decision Tree results of level of severity of $R A$}

Table 11-29: Min/Max and Median/Max Decision Tree result (Using 26 patients and 26 control subjects)

\begin{tabular}{|l|l|r|r|r|r|r|}
\hline \multicolumn{2}{|l|}{ DT\# } & 1 & 2 & 3 & 4 & 5 \\
\hline \multirow{4}{*}{ Train } & Sensitivity & 100 & 100 & 95.2 & 86.9 & 90.3 \\
& Specificity & 85.5 & 84.9 & 86.9 & 85.5 & 85.5 \\
& CCR & 86.3 & 86.0 & 85.1 & 84.2 & 82.5 \\
\hline \multirow{5}{*}{ Test } & Sensitivity & 92.1 & 92.1 & 87.3 & 78.8 & 85.2 \\
& Specificity & 78.6 & 72.9 & 63.3 & 67.6 & 80.1 \\
& CCR & 80.3 & 77.5 & 70.3 & 68.2 & 82.7 \\
\hline
\end{tabular}

Table 11-30: Median/Max and Mode/Min Decision Tree result (Using 26 patients and 26 control subjects)

\begin{tabular}{|r|r|r|r|r|r|r|}
\hline \multicolumn{2}{|l|}{ DT\# } & 1 & 2 & 3 & 4 & 5 \\
\hline \multirow{4}{*}{ Train } & Sensitivity & 100 & 100 & 87.4 & 92.3 & 92.3 \\
& Specificity & 89.5 & 85.5 & 80.6 & 85.5 & 85.5 \\
& & 90.8 & 88.8 & 80.0 & 88.9 & 88.9 \\
\hline \multirow{4}{*}{ Test } & Sensitivity & 90.0 & 88.0 & 90.0 & 90.0 & 90.0 \\
& Specificity & 85.3 & 77.9 & 85.3 & 78.4 & 80.6 \\
& CCR & 86.6 & 80.9 & 86.6 & 78.2 & 85.4 \\
\hline
\end{tabular}


Table 11-31: Min/Max and Median/Min Decision Tree result (Using 26 patients and 26 control subjects)

\begin{tabular}{|l|l|r|r|r|r|r|}
\hline \multicolumn{2}{|l|}{ DT\# } & 1 & 2 & 3 & 4 & 5 \\
\hline \multirow{4}{*}{ Train } & Sensitivity & 100 & 100 & 100.0 & 86.4 & 95.3 \\
& Specificity & 83.3 & 73.3 & 83.3 & 85.5 & 86.4 \\
& CCR & 84.4 & 76.9 & 82.1 & 86.2 & 78.9 \\
\hline \multirow{5}{*}{ Test } & Sensitivity & 88.1 & 88.1 & 88.3 & 88.1 & 88.3 \\
& Specificity & 80.2 & 82.4 & 75.6 & 80.1 & 67.3 \\
& CCR & 83.6 & 80.1 & 78.4 & 80.2 & 77.8 \\
\hline
\end{tabular}

Table 11-32: Min/Max and Mode/Min Decision Tree result (Using 26 patients and 26 control subjects)

\begin{tabular}{|c|l|r|r|r|r|r|}
\hline \multicolumn{2}{|l|}{ DT\# } & 1 & 2 & 3 & 4 & 5 \\
\hline \multirow{4}{*}{ Train } & Sensitivity & 100 & 89.8 & 84.4 & 96.7 & 90.3 \\
& Specificity & 96.3 & 87.4 & 87.4 & 80.5 & 80.8 \\
& CCR & 96.2 & 88.6 & 80.9 & 88.6 & 86.6 \\
\hline \multirow{5}{*}{ Test } & Sensitivity & 86.2 & 78.6 & 82.1 & 78.8 & 82.1 \\
& Specificity & 80.2 & 76.9 & 67.5 & 64.1 & 80.6 \\
& SPC & 80.2 & 75.8 & 74.8 & 75.5 & 78.4 \\
\hline
\end{tabular}


Patient \# Imaging Date Rheumatologist's Evaluation

Patient 1

Patient 2

Patient 3

Patient 4

Patient 5

Patient 6

Patient 7

Patient

Patient 9

Patient 10

Patient II

Putient 12

Patient 13

Patient 14

Patient 15

Patient 16

Patient 17

Patient 18

Patient 19

Patienc 20

Patient 21

Patient 22

Patient 23

Patient 24

Patient 25

Patient 26

April 292008

April 252008

May 8008

June 122008

June 122008

June 132008

tune 132008

June 162008

June 172008

June 172008

June 192008

June 212008

June 242008

December 162010

December 162010

December 162010

Decernber 182010

December 202010

December 202010

December 202010

December 202010

Decenber 222010

January 52011

January 82011

Jamuary 122011

Jamuary 132011

Low

Low

High

Low

Low

Low

High

Low

Low

Medium

Medium

Low

Medium

Molete

Lav to modrare

Low to actoret?

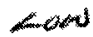

200 to 0

sisuro 0

Law to mathote

Lute to 0

2 ons

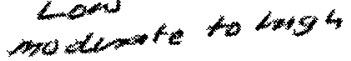

$\angle w \rightarrow 0$

$\cos x+2 x$

$\angle$ tot 0

*Note that the Evaluation of the Patients 1 through 13 was adapted from the rheumatologist"s evaluation of those patients at the time of the imaging session from previous work (Adka 2009)

Rheumajologist's Signature

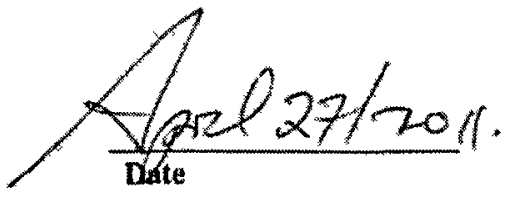

$\frac{\text { Aquil } 27,2011}{\text { Date }}$

Student Signature 


\section{APPENDIX G - Science behind Infrared Imaging}

\subsection{Electromagnetic Spectrum}

The electromagnetic spectrum is divided arbitrarily into a number of wavelength regions, called bands, distinguished by the methods used to produce and detect the radiation. Thermography makes use of the infrared spectral band. At the short-wavelength end the boundary lies at the limit of visual perception, in the deep red. At the long-wavelength end it merges with the microwave radio wavelengths, in the millimeter range. The infrared band is often subdivided into four smaller bands. They include: the near infrared $(0.75-3 \mu \mathrm{m})$, the middle infrared $(3-6 \mu \mathrm{m})$, the far infrared $(6-15 \mu \mathrm{m})$ and the extreme infrared $(15-100 \mu \mathrm{m})$ (FLIR Systems, 2007).

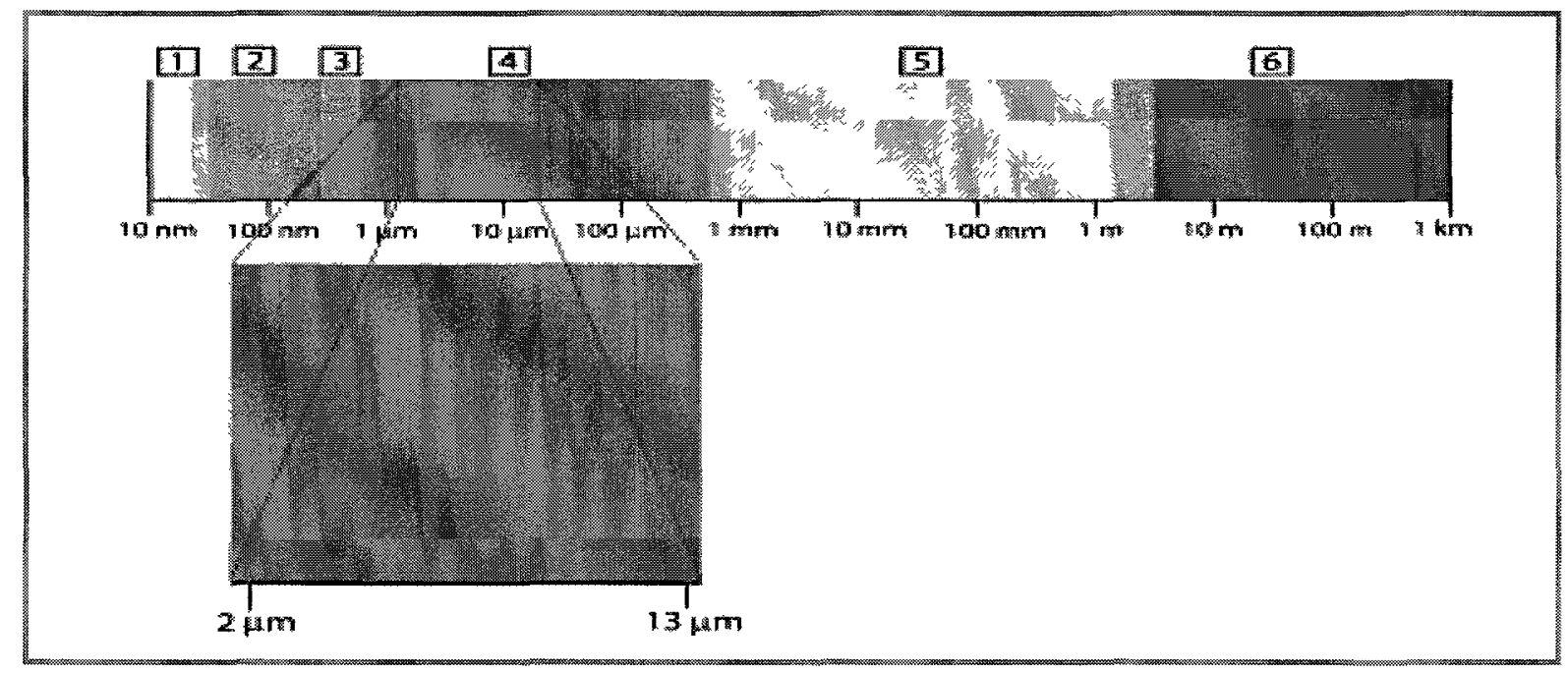

Fig. 13-1: Electromagnetic Spectrum (FLIR Systems, 2007)

Thermal infrared imaging's earliest application was for military use. This technology was developed solely for the purposes of warfare: using thermal IR cameras for night vision and human detection. Due to the nature of its very first applications, most thermal IR cameras 
function in the far infrared range $(6-15 \mu \mathrm{m})$, since the signal at these wavelengths can travel through most variations in the environment such as smoke, smog, dust, humidity.

\subsection{Blackbody Radiation}

A blackbody is defined as an object which absorbs all radiation that impinges on it at any wavelength. The apparent misnomer black relating to an object emitting radiation is explained by Kirchhoff's Law, which states that "a body capable of absorbing all radiation at any wavelength is equally capable in the emission of radiation" (FLIR Systems, 2007). The blackbody is an ideal body. It absorbs all incident radiation and is conversely a perfect radiator; this implies that a blackbody absorbs and emits the maximum theoretically possible energy at a given temperature" (Ng, 2009).

\subsection{Planck's Law}

Planck's law is used to describe the radiation emitted by a black body. Planck was able to describe the spectral distribution of the radiation from a blackbody by means of the following formula:

$$
W_{\lambda b}=\frac{2 \pi h c^{3}}{\lambda^{5}\left(e^{\frac{h c}{\lambda k T}}-1\right)} \times 10^{-6}\left[\text { Watt } / m^{2} \mu m\right]
$$

Where $W_{\lambda b}$ is the blackbody spectral radiant emittance at wavelength , $\mathrm{c}$ is the velocity of light ( $3 \times 108 \mathrm{~m} / \mathrm{s}), \mathrm{h}$ is the Planck's constant (6.626 x 10-34 Joule sec.), $\mathrm{k}$ is the Boltzmann's constant (1.380 $\times 10-23 \mathrm{Joule} / \mathrm{K}), \mathrm{T}$ is the absolute temperature $(\mathrm{K})$ of a blackbody and $\lambda$ is the wavelength $(\mu \mathrm{m})$ 
Planck's formula, when plotted graphically for various temperatures, produces a family of curves. Following any particular Planck curve, the spectral emittance is zero at $\lambda=0$, then increases rapidly to a maximum at a wavelength $\lambda_{\max }$ and after passing it approaches zero again at very long wavelengths. In essence, the higher the temperature; the shorter the wavelength at which a maximum occurs.

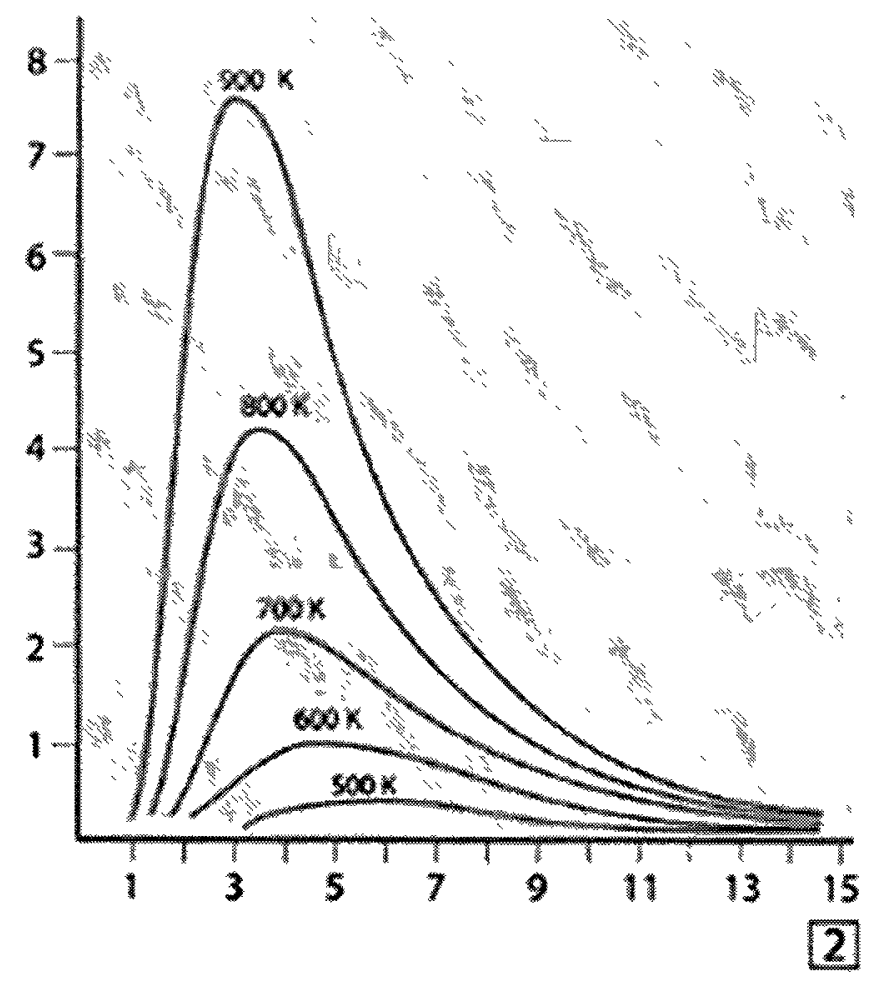

Fig. 13-2: Emissive Power of a Blackbody at Different Temperatures and Wavelengths According to Planck's Radiation Law (FLIR Systems, 2007)

\subsection{Wein's Displacement Law}

Wein's law can be expressed by differentiating the Planck's formula with respect to $\lambda$, and then finding its maximum. This is expressed in the equation below:

$$
\lambda_{\max }=\frac{2898}{T}[\mu \mathrm{m}]
$$


where $\lambda_{\max }$ is the maximum emissive power and $\mathrm{T}$ is the temperature (in $\mathrm{K}$ ).

Wien's formula expresses mathematically the common observation that colors vary from red to orange or yellow as the temperature of a thermal radiator increases. The wavelength of the color is the same as the wavelength calculated for $\lambda_{\max }$. A good approximation of the value of $\lambda_{\max }$ for a given blackbody temperature is obtained by applying the rule-of-thumb $3000 / \mathrm{T} \mu \mathrm{m}$.

At room temperature $(300 \mathrm{~K})$ the peak of radiant emittance lies at $9.7 \mu \mathrm{m}$, in the far infrared in the electromagnetic spectrum. The figure below shows the Planckian curves from $100 \mathrm{~K}$ to $1000 \mathrm{~K}$. The dotted line represents the maximum radiant emittance at each temperature according to Wien's displacement law (FLIR Systems 2007).

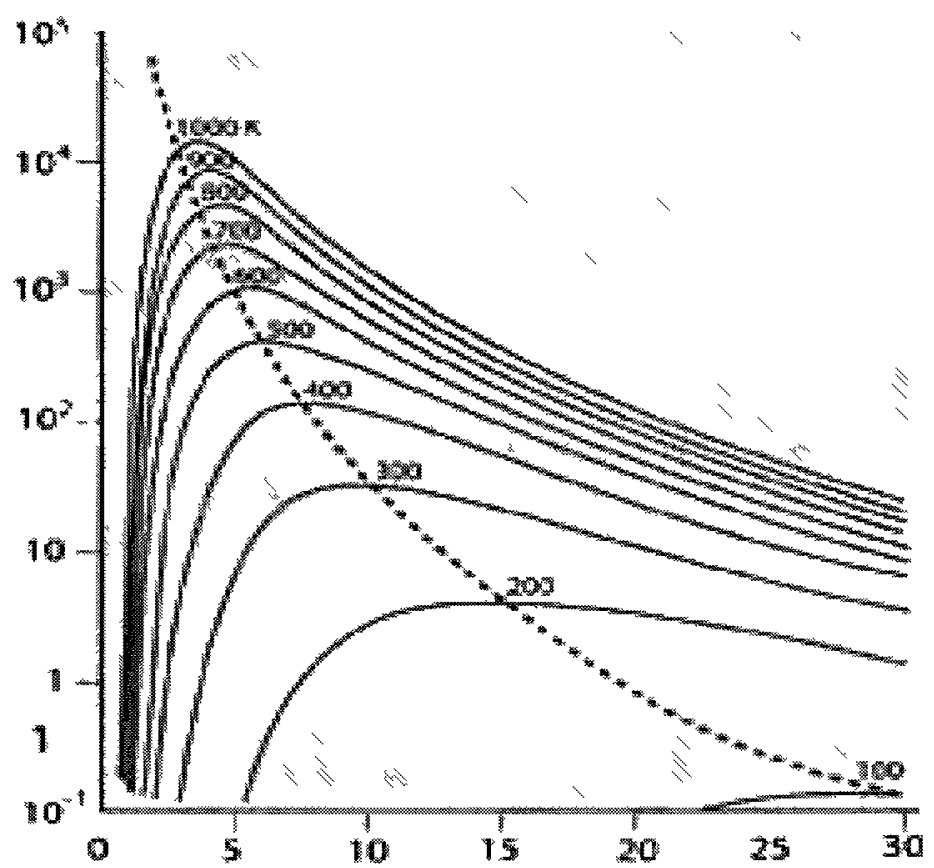

Fig. 13-3: Planckian Curves Plotted on Semilog Scale (FLIR Systems, 2007) 


\subsection{Stephan-Boltzmann Law}

Stephan-Boltzmann law is described as the total radiant emittance of a blackbody, this is expressed by integrating Planck's formula from $\lambda=0$ to $\lambda=\infty$. In other words, StefanBoltzmann formula, states that the total emissive power of a blackbody is proportional to the fourth power of its absolute temperature. Graphically, $\mathrm{W}_{\lambda \mathrm{b}}$ represents the area below the Planck curve for a particular temperature. It can be shown that the radiant emittance in the interval $\lambda=0$ to $\lambda_{\max }$ is only $25 \%$ of the total, which represents about the amount of the sun's radiation which lies inside the visible light spectrum.

Using the Stefan-Boltzmann formula to calculate the power radiated by the human body, at a temperature of $300 \mathrm{~K}$ and an external surface area of approx. $2 \mathrm{~m}^{2}$, we obtain $1 \mathrm{~kW}$. This power loss could not be sustained if it were not for the compensating absorption of radiation from surrounding surfaces, at room temperatures which do not vary too drastically from the temperature of the body - or, of course, the addition of clothing (FLIR Systems 2007). 